\title{
A Quantitative Tandem Mass Spectrometry and Scaled-Down QuEChERS Approach for Simultaneous Analysis of Pesticide Multiresidues in Human Urine
}

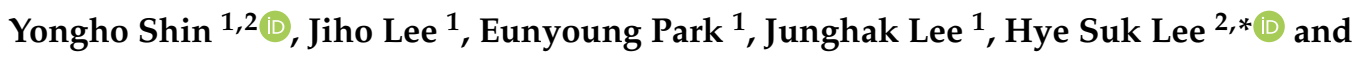 \\ Jeong-Han Kim $1, *$ (i) \\ 1 Pesticide Chemistry and Toxicology Laboratory, Department of Agricultural Biotechnology and Research \\ Institute of Agriculture and Life Sciences, Seoul National University, Seoul 08826, Korea; \\ driger6103@catholic.ac.kr (Y.S.); micai1@snu.ac.kr (J.L.); pey4321@snu.ac.kr (E.P.); crane245@snu.ac.kr (J.L.) \\ 2 Drug Metabolism and Bioanalysis Laboratory, College of Pharmacy, The Catholic University of Korea, \\ Bucheon-si, Gyeonggi-do 14662, Korea \\ * Correspondence: kjh2404@snu.ac.kr (J.-H.K.); sianalee@catholic.ac.kr (H.S.L.); Tel.: +82-2-880-4644 (J.-H.K.); \\ +82-2-2164-4061 (H.S.L.); Fax: +82-2-873-4415 (J.-H.K.); +82-32-342-2013 (H.S.L.)
}

Received: 7 March 2019; Accepted: 1 April 2019; Published: 4 April 2019

\begin{abstract}
Multiresidual pesticide determination in a biological sample is essential for an immediate decision and response related to various pesticide intoxications. A rapid and simultaneous analytical method for 260 pesticides in human urine was developed and validated using liquid chromatography-tandem mass spectrometry (LC-MS/MS). High speed positive/negative switching electrospray ionization (ESI) mode was used, and scheduled multiple reaction monitoring (MRM) was optimized. Three versions of scaled-down QuEChERS procedures were evaluated, and the procedure using non-buffer reagents (magnesium sulfate and sodium chloride) and excluding cleanup steps was selected for optimum pesticide extraction. The limit of quantitation (LOQ) in this methodology was $10 \mathrm{ng} / \mathrm{mL}$ for each target pesticide, and correlation coefficient $\left(\mathrm{r}^{2}\right)$ values of calibration curves were $\geq 0.988$ (linearity range; $10-250 \mathrm{ng} / \mathrm{mL}$ ). In accuracy and precision tests, the relative error ranges were $-18.4 \%$ to $19.5 \%$, with relative standard deviation (RSD) $2.1 \%-19.9 \%$ at an LOQ level (10 $\mathrm{ng} / \mathrm{mL}$ ), and $-14.7 \%$ to $14.9 \%$ (RSD; $0.6 \%-14.9 \%$ ) at higher concentrations (50, 150, and $250 \mathrm{ng} / \mathrm{mL}$ ). Recovery range was $54.2 \%-113.9 \%$ (RSD; $0.3 \%-20.0 \%$ ), and the soft matrix effect (range; $-20 \%$ to $20 \%$ ) was observed in $75.4 \%$ of target pesticides. The established bioanalytical methods are sufficient for application to biomonitoring in agricultural exposures and applicable in the forensic and clinic.
\end{abstract}

Keywords: pesticide; multiresidue; forensic; urine; QuEChERS; LC-MS/MS

\section{Introduction}

Pesticides have been widely applied on farms for control of problematic weeds or harmful pests such as certain insects and fungi. The use of pesticides has contributed to the improvement of crop/livestock yields and quality, increased shelf life of produce, and prevention of harmful organisms from interfering in human activities and structures, from which secondary benefits such as national agricultural economic development, reduced maintenance costs, or quality of life improvement have followed [1]. Despite the considerable advantages of pesticides, unwanted side effects have also followed. Pesticide intoxication resulting from intentional intake or misuse during cultivation is a major social problem. Gunnell and coworkers investigated the global distribution of suicide by pesticide and estimated that there are 258234 (plausible range from 233997 to 325907) suicides from pesticide poisoning each year, representing $30 \%$ (27\% to $37 \%)$ of all suicides worldwide [2]. In the United States, 234 deaths by pesticide poisoning were identified over a 10-year span (1999 to 2008) according 
to the Centers for Disease Control and Prevention's Wide-ranging Online Data for Epidemiologic Research (CDC WONDER) report, and an average of 20116 people were exposed to pesticides annually, accounting for $17.8 \%$ of treatment in health care facilities from 2006 to 2010 [3]. In the Republic of Korea, 16161 reports of mortality and 45291 reports of inpatient and outpatient treatment related to pesticide intoxication were reported during 5 years (2006 to 2010) [4].

Biological monitoring of acute or chronic pesticide poisoning is useful for identifying evidence of health problems in the environment/ecotoxicology, agricultural and forensic fields, or for detoxification in a medical institution. Human biological samples such as urine [5-9], blood [10-12], hair [13,14], and saliva [15] have been the primary sources for determination of pesticides. Among them, urine has several advantages over other samples. Urine is easier to obtain than invasive samples such as blood, and larger amounts of urine are available compared with blood, hair, and saliva. Because urine is a homogeneous biological fluid composed of $95 \%$ water [6], complex preparation steps for purification of target pesticides are not needed.

Although some pesticides are metabolized rapidly in the body and excreted in urine within $48 \mathrm{~h}$ [16], various chemical groups of pesticides still remain intact and present in urine [17-20]. It is easier and less costly to obtain analytical standards of pesticides rather than those of metabolites. Detecting as many pesticides as possible is also needed because there have been deaths resulting from various chemical groups of pesticides [21], some of them (e.g., benzoximate and etofenprox) showing very low acute toxicity $\left(\mathrm{LD}_{50}>10,000 \mathrm{mg} / \mathrm{kg}\right.$; oral acute for rat) [22].

For multiresidual pesticide analysis, mass spectrometry coupled with chromatography is widely utilized. A single quadrupole (SQ) mass filter has a high scan speed technique using selected ion monitoring (SIM), therefore it can determine more than one hundred of target compounds simultaneously. However, the SQ is not able to distinguish an analyte from other analytes or interferences with overlapped retention time $\left(t_{R}\right)$ and the same mass to charge ratio $(\mathrm{m} / \mathrm{z})$. A high-resolution mass spectrometry has an excellent selectivity useful for screening target and non-target compounds, whereas it has limitations on targeted multiresidue determination due to its low scan speed. With the introduction of triple quadrupole (TQ) mass filters, analysis of more than 200 target compounds at once became feasible [23]. The multiple reaction monitoring (MRM) mode of the TQ can select and detect target pesticides with high-throughput and selectivity.

The aqueous characteristic of a urine and advanced separation techniques such as LC-MS/MS make urine preparation relatively convenient and easy. Direct injection [6] or dilute-and-shoot [24,25] procedures are the simplest ways to identify pesticides in urine. Nevertheless, these processes have major problems in that urinary salts or macromolecules may decrease the sensitivity of an instrument or cause severe clogging on the injection syringe or ESI probe. Solid phase extraction (SPE) $[5,26,27]$ and liquid-liquid extraction (LLE) [28] have been popular preparation methods for pesticide extraction. However, these procedures also have disadvantages. SPE is not useful for multiresidue analysis due to the difficulty of finding optimum washing/elution conditions covering various chemical properties. LLE is simpler and easier than SPE, but interferences from urine may remain in the extract and cause a serious matrix effect or lead to low extraction efficiency. The Quick, Easy, Cheap, Effective, Rugged, and Safe (QuEChERS) method introduced by Anastassiades and coworkers is one of the most effective methodologies overcoming the limits or drawbacks of other preparations [29]. The original QuEChERS optimized for crop samples has been modified according to analytical situations [30-33] and extended for matrix materials such as biological samples [34-36].

The aim of this study is to develop and validate a reliable quantitative method for 260 pesticides in human urine utilizing liquid chromatography-tandem mass spectrometry (LC-MS/MS). The high speed switchable positive/negative ESI mode of LC-MS/MS is used, and scheduled MRM has been established for each pesticide to analyze within a short time of $15 \mathrm{~min}$. For effective and rugged multiresidue extraction, three different versions of QuEChERS were tried and the optimum procedure was established. This analytical method would be applicable for the monitoring of pesticide 
multiresidues in urinary samples in environmental, agricultural and forensic science facilities or in hospitals for clinical purposes.

\section{Results and Discussion}

\subsection{Recovery of Three Versions of Urine Preparation Methods}

Urine is a liquid sample, so it is appropriate to prepare the sample using simple QuEChERS methods for high extraction efficiency (recovery) of pesticide multiresidues. Since the first QuEChERS method for crops was developed using GC-MS in 2003 [29], there have been preparation procedure improvements for LC-amenable pesticides or lower recovery rate compounds such as $\mathrm{pH}$-dependent pesticides [37,38]. In this study, optimization of the final preparation step was established by comparing the scaled-down methods from three representative QuEChERS procedures [29-31]. During the extraction procedure, the sample was cooled in an ice bath to prevent pesticide degradation by the heat generated from the reaction between $\mathrm{MgSO}_{4}$ and water. Because the volume of urine in the preparation was very small $(100 \mu \mathrm{L})$, the extraction solvent volume and the quantity of QuEChERS partitioning reagents were also reduced (Table 1$)$.

Table 1. Reagents used in three versions of the urine treatment and distribution of recovery results.

\begin{tabular}{|c|c|c|c|}
\hline Item & Method A & Method B & Method C \\
\hline \multicolumn{4}{|l|}{ Reagents } \\
\hline Extraction Solvent & $400 \mu \mathrm{L}$ of acetonitrile & $1 \%$ HOAc in acetonitrile $(400 \mu \mathrm{L})$ & $400 \mu \mathrm{L}$ of acetonitrile \\
\hline & & & $\mathrm{MgSO}_{4}(40 \mathrm{mg})$ \\
\hline \multirow{3}{*}{ Extraction Reagent } & $\mathrm{MgSO}_{4}(40 \mathrm{mg})$ & $\mathrm{MgSO}_{4}(40 \mathrm{mg})$ & $\mathrm{NaCl}(10 \mathrm{mg})$ \\
\hline & $\mathrm{NaCl}(10 \mathrm{mg})$ & $\mathrm{NaOAc}(10 \mathrm{mg})$ & $\mathrm{Na}_{3}$ Citrate $(10 \mathrm{mg})$ \\
\hline & & & $\mathrm{Na}_{2} \mathrm{HCitr}(5 \mathrm{mg})$ \\
\hline Recovery (\%) & No. of Analytes (\%) & No. of Analytes (\%) & No. of Analytes (\%) \\
\hline $40-70(\mathrm{RSD} \leq 20 \%)$ & $2(0.8 \%)$ & $3(1.2 \%)$ & 0 \\
\hline $70-120(\mathrm{RSD} \leq 20 \%)$ & $258(99.2 \%)$ & $256(98.5 \%)$ & $258(99.2 \%)$ \\
\hline $70-120$ (RSD > 20\%) & 0 & 0 & $1(0.4 \%)$ \\
\hline$>120(\mathrm{RSD} \leq 20 \%)$ & 0 & $1(0.4 \%)$ & 0 \\
\hline n.d. ${ }^{-}$ & 0 & 0 & $1(0.4 \%)$ \\
\hline Sum & $260(100 \%)$ & $260(100 \%)$ & $260(100 \%)$ \\
\hline Average Recovery & $92.3 \%$ & $87.3 \%$ & $84.9 \%$ \\
\hline
\end{tabular}

To verify the recoveries for methods $\mathrm{A}, \mathrm{B}$, and C, each peak area of a pre- and a post-spiked (matrix-matched) samples at $250 \mathrm{ng} / \mathrm{mL}$ was compared. In accordance with SANTE/11813/2017 guidelines [39], the number of pesticides satisfying the recovery range of $70 \%-120 \%$ and relative standard deviation (RSD) $\leq 20 \%$ was verified for the 260 compounds. The results showed that $99.2 \%$ (A), 98.5\% (B), and 99.2\% (C) of total pesticides satisfied the criteria, respectively (Table 1 and Table S1). Method A and C showed slightly more compounds satisfying the criteria than method B. Method A showed consistent repeatability (RSD $\leq 20 \%$ ) in all compounds. In method C, however, cartap was not recovered at all (Table S1). Average recovery of method A was also superior $(92.3 \%)$ to those of methods B $(87.3 \%)$ and C (84.9\%).

\subsection{Relative Peak Intensity of Three Versions of Urine Preparation Methods}

The relative peak intensity of each method was verified to compare a post-spiked sample and a solvent (non-matrix contained) standard at 10 and $250 \mathrm{ng} / \mathrm{mL}$ (Table S2). When the area value of each pesticide peak in the solvent standard is converted to 100, a relative peak intensity under/above 100 means that the signal is suppressed/enhanced by urine matrices. Relative area range distribution showed that method A had the largest numbers of compounds ( $72.7 \%$ and $74.6 \%$ of 260 compounds) within $80-120$ than method B (57.7\% and $60.4 \%)$ and C (47.3\% and $48.5 \%)$ at both concentrations (Table 2). The results indicate that matrix effects (especially signal suppression) were much reduced in 
method A than B and C. The first reason is that method A used only two types of reagents $\left(\mathrm{MgSO}_{4}\right.$ and $\mathrm{NaCl}$ ) and no acids or buffers (Table 1), so matrix effect from the reagents themselves could be minimized. The other reason is that acidic or buffer reagents in method $\mathrm{B}$ and $\mathrm{C}$ extracted different kinds of matrices or excessive unnecessary matrices than those in method $\mathrm{A}$, thus these matrices may increase matrix effects.

Table 2. Distribution of relative peak intensity results (100 at solvent standard) for three versions of the urine treatment.

\begin{tabular}{|c|c|c|c|c|c|c|}
\hline \multirow{3}{*}{$\begin{array}{l}\text { Relative Peak } \\
\text { Intensity }^{1}\end{array}$} & \multirow{2}{*}{\multicolumn{2}{|c|}{$\frac{\text { Method A }}{\text { No. of Analytes (\%) }}$}} & \multirow{2}{*}{\multicolumn{2}{|c|}{$\begin{array}{c}\text { Method B } \\
\text { No. of Analytes (\%) }\end{array}$}} & \multirow{2}{*}{\multicolumn{2}{|c|}{$\begin{array}{c}\text { Method C } \\
\text { No. of Analytes (\%) }\end{array}$}} \\
\hline & & & & & & \\
\hline & $10 \mathrm{ng} / \mathrm{mL}$ & $250 \mathrm{ng} / \mathrm{mL}$ & $10 \mathrm{ng} / \mathrm{mL}$ & $250 \mathrm{ng} / \mathrm{mL}$ & $10 \mathrm{ng} / \mathrm{mL}$ & $250 \mathrm{ng} / \mathrm{mL}$ \\
\hline$<50$ & $7(2.7 \%)$ & $6(2.3 \%)$ & $25(9.6 \%)$ & $21(8.1 \%)$ & 45 (17.3\%) & $44(16.9 \%)$ \\
\hline $50-80$ & 46 (17.7\%) & $43(16.5 \%)$ & $71(27.3 \%)$ & 73 (28.1\%) & 77 (29.6\%) & $78(30.0 \%)$ \\
\hline $80-120$ & 189 (72.7\%) & $194(74.6 \%)$ & $150(57.7 \%)$ & 157 (60.4\%) & $123(47.3 \%)$ & $126(48.5 \%)$ \\
\hline $120-150$ & $15(5.8 \%)$ & $15(5.8 \%)$ & $13(5.0 \%)$ & $8(3.1 \%)$ & $13(5.0 \%)$ & $9(3.5 \%)$ \\
\hline$>150$ & $3(1.2 \%)$ & $2(0.8 \%)$ & $1(0.4 \%)$ & $1(0.4 \%)$ & $1(0.4 \%)$ & $2(0.8 \%)$ \\
\hline n.d. ${ }^{2}$ & 0 & 0 & 0 & 0 & $1(0.4 \%)$ & $1(0.4 \%)$ \\
\hline Sum & $260(100 \%)$ & $260(100 \%)$ & $260(100 \%)$ & $260(100 \%)$ & $260(100 \%)$ & $260(100 \%)$ \\
\hline
\end{tabular}

From the recovery and the intensity results, method A was selected as the final procedure for extraction and partitioning. Cleanup procedures such as dispersive SPE (dSPE) were excluded to prevent the loss of labile target compounds and to minimize the preparation time.

Analytical conditions (retention times, MRM transitions, and ratio) were shown in Table 3, and total ion chromatograms (TICs) of 260 pesticides in urine are given in Figure 1.

Table 3. Retention time $\left(t_{R}\right)$, monoisotopic mass, and MRM transition of 260 target pesticides.

\begin{tabular}{|c|c|c|c|c|c|c|c|}
\hline \multirow{2}{*}{ No. } & \multirow{2}{*}{ Compound Name } & \multirow{2}{*}{$t_{R}(\min )$} & \multirow{2}{*}{$\begin{array}{l}\text { Mono-Isotopic } \\
\text { Mass }\end{array}$} & \multirow{2}{*}{$\begin{array}{c}\text { Ionization } \\
\text { Form of } \\
\text { Precursor Ion }\end{array}$} & \multicolumn{2}{|c|}{ Precursor Ion > Product Ion $\left(\mathrm{CE}^{1}, \mathrm{~V}\right)$} & \multirow{2}{*}{ Ratio $^{2}(\%)$} \\
\hline & & & & & Quantifier & Qualifier & \\
\hline 1 & Acetamiprid & 3.26 & 222.1 & {$[\mathrm{M}+\mathrm{H}]^{+}$} & $222.6>126.0(-20)$ & $222.6>56.1(-15)$ & 36.1 \\
\hline 2 & Alachlor & 8.03 & 269.1 & {$[\mathrm{M}+\mathrm{H}]^{+}$} & $269.7>238.0(-11)$ & $269.7>162.1(-20)$ & 101.9 \\
\hline 3 & Allidochlor & 3.76 & 173.1 & {$[\mathrm{M}+\mathrm{H}]^{+}$} & $173.9>98.1(-14)$ & $173.9>41.1(-24)$ & 53.5 \\
\hline 4 & Ametoctradin & 7.22 & 275.2 & {$[\mathrm{M}+\mathrm{H}]^{+}$} & $275.7>176.1(-36)$ & $275.7>149.1(-37)$ & 107.4 \\
\hline 5 & Ametryn & 4.94 & 227.1 & {$[\mathrm{M}+\mathrm{H}]^{+}$} & $227.6>186.0(-19)$ & $227.6>68.0(-38)$ & 55.5 \\
\hline 6 & Amisulbrom & 7.49 & 465.0 & {$[\mathrm{M}+\mathrm{H}]^{+}$} & $465.7>226.9(-21)$ & $465.7>148.0(-51)$ & 37.7 \\
\hline 7 & Anilofos & 6.57 & 367.0 & {$[\mathrm{M}+\mathrm{H}]^{+}$} & $367.5>125.0(-31)$ & $367.5>198.9(-15)$ & 69.0 \\
\hline 8 & Asulam & 2.88 & 230.0 & {$[\mathrm{M}+\mathrm{H}]^{+}$} & $231.0>155.9(-11)$ & $231.0>92.1(-24)$ & 64.1 \\
\hline 9 & Atrazine & 4.66 & 215.1 & {$[\mathrm{M}+\mathrm{H}]^{+}$} & $215.7>174.0(-18)$ & $215.7>104.0(-28)$ & 42.5 \\
\hline 10 & Azaconazole & 4.71 & 299.0 & {$[\mathrm{M}+\mathrm{H}]^{+}$} & $299.5>158.9(-27)$ & $299.5>230.9(-17)$ & 38.0 \\
\hline 11 & Azamethiphos & 3.80 & 324.0 & {$[\mathrm{M}+\mathrm{H}]^{+}$} & $324.5>183.0(-16)$ & $324.5>112.1(-36)$ & 86.6 \\
\hline 12 & Azimsulfuron & 4.53 & 424.1 & {$[\mathrm{M}+\mathrm{H}]^{+}$} & $424.6>182.0(-19)$ & $424.6>139.0(-41)$ & 29.5 \\
\hline 13 & Azinphos-methyl & 4.95 & 317.0 & {$[\mathrm{M}+\mathrm{H}]^{+}$} & $317.8>77.0(-39)$ & $317.8>125.0(-20)$ & 53.6 \\
\hline 14 & Azoxystrobin & 5.03 & 403.1 & {$[\mathrm{M}+\mathrm{H}]^{+}$} & $403.6>372.0(-17)$ & $403.6>344.0(-25)$ & 29.0 \\
\hline 15 & Bendiocarb & 3.95 & 223.1 & {$[\mathrm{M}+\mathrm{H}]^{+}$} & $223.6>109.0(-18)$ & $223.6>167.1(-10)$ & 48.4 \\
\hline 16 & Bensulfuron-methyl & 4.85 & 410.1 & {$[\mathrm{M}+\mathrm{H}]^{+}$} & $410.6>182.0(-20)$ & $410.6>149.0(-20)$ & 53.4 \\
\hline 17 & Bentazone & 3.73 & 240.1 & {$[\mathrm{M}-\mathrm{H}]^{-}$} & $238.9>132.0(24)$ & $238.9>197.1(19)$ & 59.6 \\
\hline 18 & Benthiavalicarb-isopropyl & 5.59 & 381.2 & {$[\mathrm{M}+\mathrm{H}]^{+}$} & $381.6>180.0(-33)$ & $381.6>116.1(-21)$ & 48.9 \\
\hline 19 & Benzobicyclon & 5.45 & 446.0 & {$[\mathrm{M}+\mathrm{H}]^{+}$} & $446.5>257.0(-24)$ & $446.5>229.0(-36)$ & 72.5 \\
\hline 20 & Benzoximate & 7.01 & 363.1 & {$[\mathrm{M}+\mathrm{H}]^{+}$} & $363.9>199.0(-12)$ & $363.9>105.1(-26)$ & 51.9 \\
\hline 21 & Boscalid & 5.31 & 342.0 & {$[\mathrm{M}+\mathrm{H}]^{+}$} & $342.6>307.0(-21)$ & $342.6>140.0(-20)$ & 31.0 \\
\hline 22 & Bromacil & 4.02 & 260.0 & {$[\mathrm{M}+\mathrm{H}]^{+}$} & $260.8>204.9(-14)$ & $260.8>187.9(-28)$ & 22.3 \\
\hline 23 & Bromobutide & 6.06 & 311.1 & {$[\mathrm{M}+\mathrm{H}]^{+}$} & $311.8>194.0(-13)$ & 311.8 > $91.1(-45)$ & 73.0 \\
\hline 24 & Bupirimate & 6.06 & 316.2 & {$[\mathrm{M}+\mathrm{H}]^{+}$} & $316.6>166.1(-24)$ & $316.6>210.1(-24)$ & 29.2 \\
\hline 25 & Buprofezin & 7.86 & 305.2 & {$[\mathrm{M}+\mathrm{H}]^{+}$} & 305.7 > $57.1(-24)$ & $305.7>200.9(-15)$ & 80.4 \\
\hline 26 & Butachlor & 7.98 & 311.2 & {$[\mathrm{M}+\mathrm{H}]^{+}$} & $312.1>238.1(-13)$ & $312.1>162.1(-21)$ & 36.6 \\
\hline 27 & Butafenacil & 5.81 & 474.1 & {$\left[\mathrm{M}+\mathrm{NH}_{4}\right]^{+}$} & $491.6>331.0(-25)$ & $491.6>180.0(-45)$ & 76.6 \\
\hline 28 & Cadusafos & 7.22 & 270.1 & {$[\mathrm{M}+\mathrm{H}]^{+}$} & $270.6>158.9(-17)$ & $270.6>130.9(-22)$ & 111.3 \\
\hline 29 & Carbaryl & 4.14 & 201.1 & {$[\mathrm{M}+\mathrm{H}]^{+}$} & $201.8>145.1(-11)$ & $201.8>127.1(-26)$ & 73.3 \\
\hline 30 & Carbendazim & 3.11 & 191.1 & {$[\mathrm{M}+\mathrm{H}]^{+}$} & $191.6>159.8(-24)$ & $191.6>132.1(-29)$ & 42.2 \\
\hline 31 & Carbofuran & 3.98 & 221.1 & {$[\mathrm{M}+\mathrm{H}]^{+}$} & $221.6>123.0(-21)$ & $221.6>165.1(-11)$ & 70.6 \\
\hline
\end{tabular}


Table 3. Cont.

\begin{tabular}{|c|c|c|c|c|c|c|c|}
\hline \multirow{2}{*}{ No. } & \multirow{2}{*}{ Compound Name } & \multirow{2}{*}{$t_{R}(\min )$} & \multirow{2}{*}{$\begin{array}{l}\text { Mono-Isotopic } \\
\text { Mass }\end{array}$} & \multirow{2}{*}{$\begin{array}{c}\text { Ionization } \\
\text { Form of } \\
\text { Precursor Ion }\end{array}$} & Precursor Ion > & luct Ion $\left(\mathrm{CE}^{1}, \mathrm{~V}\right)$ & Batio $^{2}$ (o) \\
\hline & & & & & Quantifier & Qualifier & Kat1o $^{-}(\%)$ \\
\hline 32 & Carbophenothion & 8.43 & 342.0 & {$[\mathrm{M}+\mathrm{H}]^{+}$} & $342.8>157.0(-13)$ & $342.8>45.0(-37)$ & 34.3 \\
\hline 33 & Carboxin & 4.17 & 235.1 & {$[\mathrm{M}+\mathrm{H}]^{+}$} & $235.6>143.0(-15)$ & $235.6>87.0(-25)$ & 36.1 \\
\hline 34 & Carpropamid & 6.66 & 333.0 & {$[\mathrm{M}+\mathrm{H}]^{+}$} & $333.6>139.0(-21)$ & $333.6>103.1(-42)$ & 84.6 \\
\hline 35 & Cartap & 6.51 & 237.1 & {$[\mathrm{M}+\mathrm{H}]^{+}$} & $238.0>150.0(-14)$ & $238.0>73.0(-28)$ & 72.8 \\
\hline 36 & Chlorantraniliprole & 4.82 & 483.0 & {$[\mathrm{M}+\mathrm{H}]^{+}$} & $483.5>452.8(-18)$ & $483.5>285.9(-16)$ & 68.2 \\
\hline 37 & Chlorfenvinphos & 6.70 & 358.0 & {$[\mathrm{M}+\mathrm{H}]^{+}$} & $358.5>99.0(-30)$ & $358.5>169.9(-40)$ & 114.7 \\
\hline 38 & Chlorfluazuron & 8.74 & 539.0 & {$[\mathrm{M}+\mathrm{H}]^{+}$} & $539.8>382.8(-23)$ & $539.8>158.0(-20)$ & 43.2 \\
\hline 39 & Chloridazon & 3.35 & 221.0 & {$[\mathrm{M}+\mathrm{H}]^{+}$} & $221.5>104.1(-22)$ & $221.5>77.0(-35)$ & 91.1 \\
\hline 40 & Chlorotoluron & 4.44 & 212.1 & {$[\mathrm{M}+\mathrm{H}]^{+}$} & $212.7>72.1(-22)$ & $212.7>46.2(-16)$ & 52.7 \\
\hline 41 & Chlorsulfuron & 4.05 & 357.0 & {$[\mathrm{M}+\mathrm{H}]^{+}$} & $358.0>141.1(-20)$ & $358.0>167.1(-19)$ & 94.7 \\
\hline 42 & Chromafenozide & 5.87 & 394.2 & {$[\mathrm{M}+\mathrm{H}]^{+}$} & $394.8>175.1(-19)$ & $394.8>147.0(-44)$ & 18.2 \\
\hline 43 & Cinmethylin & 7.95 & 274.2 & {$\left[\mathrm{M}+\mathrm{NH}_{4}\right]^{+}$} & $292.1>105.0(-23)$ & $292.1>153.0(-11)$ & 9.7 \\
\hline 44 & Clofentezine & 7.05 & 302.0 & {$[\mathrm{M}+\mathrm{H}]^{+}$} & $303.0>138.0(-15)$ & $303.0>102.1(-35)$ & 84.9 \\
\hline 45 & Clomazone & 5.02 & 239.1 & {$[\mathrm{M}+\mathrm{H}]^{+}$} & $239.6>125.0(-20)$ & $239.6>89.1(-49)$ & 20.8 \\
\hline 46 & Clothianidin & 3.19 & 249.0 & {$[\mathrm{M}+\mathrm{H}]^{+}$} & $250.0>169.0(-13)$ & $250.0>132.0(-18)$ & 93.8 \\
\hline 47 & Cyanazine & 3.78 & 240.1 & {$[\mathrm{M}+\mathrm{H}]^{+}$} & $240.8>214.1(-17)$ & $240.8>104.0(-30)$ & 28.0 \\
\hline 48 & Cyazofamid & 6.01 & 324.0 & {$[\mathrm{M}+\mathrm{H}]^{+}$} & $325.0>108.0(-13)$ & $325.0>44.1(-31)$ & 20.8 \\
\hline 49 & Cyclosulfamuron & 5.76 & 421.1 & {$[\mathrm{M}+\mathrm{H}]^{+}$} & $421.6>261.0(-19)$ & $421.6>218.0(-26)$ & 77.4 \\
\hline 50 & Cymoxanil & 3.41 & 198.1 & {$[\mathrm{M}+\mathrm{H}]^{+}$} & $198.9>128.1(-10)$ & $198.9>111.1(-18)$ & 80.8 \\
\hline $51-1$ & Cyproconazole 1 & 5.55 & 291.1 & {$[\mathrm{M}+\mathrm{H}]^{+}$} & $291.8>70.1(-21)$ & $291.8>125.0(-31)$ & 50.8 \\
\hline $51-2$ & Cyproconazole_2 & 5.77 & 291.1 & {$[\mathrm{M}+\mathrm{H}]^{+}$} & $291.8>70.1(-21)$ & $291.8>125.0(-31)$ & 77.8 \\
\hline 52 & Cyprodinil & 6.42 & 225.1 & {$[\mathrm{M}+\mathrm{H}]^{+}$} & $225.6>93.1(-34)$ & $225.6>77.1(-45)$ & 56.4 \\
\hline 53 & Daimuron & 5.61 & 268.2 & {$[\mathrm{M}+\mathrm{H}]^{+}$} & $269.0>151.0(-20)$ & $269.0>91.0(-50)$ & 98.8 \\
\hline 54 & Diazinon & 6.77 & 304.1 & {$[\mathrm{M}+\mathrm{H}]^{+}$} & $305.0>169.0(-25)$ & $305.0>153.0(-25)$ & 73.6 \\
\hline 55 & Dicrotophos & 3.08 & 237.1 & {$[\mathrm{M}+\mathrm{H}]^{+}$} & $238.0>72.0(-30)$ & $238.0>112.1(-15)$ & 89.0 \\
\hline 56 & Diethofencarb & 5.14 & 267.1 & {$[\mathrm{M}+\mathrm{H}]^{+}$} & $268.0>124.0(-35)$ & $268.0>152.0(-25)$ & 69.9 \\
\hline 57 & Difenoconazole & 7.14 & 405.1 & {$[\mathrm{M}+\mathrm{H}]^{+}$} & $406.0>250.9(-30)$ & $406.0>188.0(-46)$ & 25.0 \\
\hline 58 & Diflubenzuron & 6.18 & 310.0 & {$[\mathrm{M}+\mathrm{H}]^{+}$} & $311.0>158.0(-14)$ & $311.0>141.0(-30)$ & 115.2 \\
\hline 59 & Diflufenican & 7.37 & 394.1 & {$[\mathrm{M}+\mathrm{H}]^{+}$} & $395.0>265.9(-25)$ & $395.0>246.0(-35)$ & 17.5 \\
\hline 60 & Dimethachlor & 4.85 & 255.1 & {$[\mathrm{M}+\mathrm{H}]^{+}$} & $256.0>224.0(-20)$ & $256.0>148.1(-30)$ & 100.9 \\
\hline 61 & Dimethametryn & 6.22 & 255.2 & {$[\mathrm{M}+\mathrm{H}]^{+}$} & $256.0>186.0(-25)$ & $256.0>68.0(-46)$ & 31.5 \\
\hline 62 & Dimethenamid & 5.35 & 275.1 & {$[\mathrm{M}+\mathrm{H}]^{+}$} & $276.0>244.0(-20)$ & $276.0>168.1(-28)$ & 48.3 \\
\hline 63 & Dimethoate & 3.30 & 229.0 & {$[\mathrm{M}+\mathrm{H}]^{+}$} & $230.0>198.9(-11)$ & $230.0>125.0(-22)$ & 112.5 \\
\hline $64-1$ & Dimethomorph_1 & 5.19 & 387.1 & {$[\mathrm{M}+\mathrm{H}]^{+}$} & $388.0>300.9(-25)$ & $388.0>165.0(-35)$ & 79.8 \\
\hline $64-2$ & Dimethomorph_2 & 5.45 & 387.1 & {$[\mathrm{M}+\mathrm{H}]^{+}$} & $388.0>300.9(-25)$ & $388.0>165.0(-35)$ & 77.7 \\
\hline 65 & Diniconazole & 7.09 & 325.1 & {$[\mathrm{M}+\mathrm{H}]^{+}$} & $326.0>70.1(-25)$ & $326.0>158.9(-33)$ & 13.1 \\
\hline 66 & Diphenamid & 4.82 & 239.1 & {$[\mathrm{M}+\mathrm{H}]^{+}$} & $240.0>134.1(-25)$ & $240.0>167.1(-22)$ & 20.4 \\
\hline 67 & Diuron & 4.72 & 232.0 & {$[\mathrm{M}+\mathrm{H}]^{+}$} & $233.0>72.0(-25)$ & $233.0>46.1(-17)$ & 51.5 \\
\hline 68 & Edifenphos & 6.57 & 310.0 & {$[\mathrm{M}+\mathrm{H}]^{+}$} & $311.0>109.0(-40)$ & $311.0>282.9(-18)$ & 71.3 \\
\hline 69 & Emamectin B1a & 7.91 & 885.5 & {$[\mathrm{M}+\mathrm{H}]^{+}$} & $886.4>158.1(-39)$ & $886.4>82.2(-55)$ & 17.4 \\
\hline 70 & Emamectin B1b & 7.60 & 871.5 & {$[\mathrm{M}+\mathrm{H}]^{+}$} & $872.3>158.1(-36)$ & $872.3>82.1(-55)$ & 17.4 \\
\hline 71 & EPN & 7.27 & 323.0 & {$[\mathrm{M}+\mathrm{H}]^{+}$} & $324.0>295.9(-14)$ & $324.0>156.9(-22)$ & 136.6 \\
\hline 72 & Epoxiconazole & 6.00 & 329.1 & {$[\mathrm{M}+\mathrm{H}]^{+}$} & $330.0>121.0(-21)$ & $330.0>101.1(-49)$ & 65.1 \\
\hline 73 & Ethaboxam (EBX) & 4.29 & 320.1 & {$[\mathrm{M}+\mathrm{H}]^{+}$} & $320.6>183.0(-21)$ & $320.6>200.0(-25)$ & 21.4 \\
\hline 74 & Ethametsulfuron-methyl & 4.16 & 410.1 & {$[\mathrm{M}+\mathrm{H}]^{+}$} & $410.6>196.0(-18)$ & $410.6>168.0(-30)$ & 76.8 \\
\hline 75 & Ethiofencarb & 4.30 & 225.1 & {$[\mathrm{M}+\mathrm{H}]^{+}$} & $225.6>107.1(-16)$ & $225.6>77.0(-45)$ & 41.6 \\
\hline 76 & Ethion & 8.06 & 384.0 & {$[\mathrm{M}+\mathrm{H}]^{+}$} & $384.6>198.9(-11)$ & $384.6>143.0(-24)$ & 108.9 \\
\hline 77 & Ethoprophos & 6.04 & 242.1 & {$[\mathrm{M}+\mathrm{H}]^{+}$} & $242.6>97.0(-32)$ & $242.6>130.9(-20)$ & 82.6 \\
\hline 78 & Ethoxyquin & 5.08 & 217.1 & {$[\mathrm{M}+\mathrm{H}]^{+}$} & $218.0>174.1(-27)$ & $218.0>148.0(-22)$ & 71.9 \\
\hline 79 & Ethoxysulfuron & 5.52 & 398.1 & {$[\mathrm{M}+\mathrm{H}]^{+}$} & $398.7>260.9(-16)$ & $398.7>218.0(-25)$ & 76.1 \\
\hline 80 & Etoxazole & 8.48 & 359.2 & {$[\mathrm{M}+\mathrm{H}]^{+}$} & $359.6>141.0(-29)$ & $359.6>113.0(-55)$ & 41.1 \\
\hline 81 & Etrimfos & 6.65 & 292.1 & {$[\mathrm{M}+\mathrm{H}]^{+}$} & $292.6>125.0(-25)$ & $292.6>265.0(-17)$ & 104.7 \\
\hline 82 & Fenamidone & 5.25 & 311.1 & {$[\mathrm{M}+\mathrm{H}]^{+}$} & $311.7>92.1(-24)$ & $311.7>236.1(-15)$ & 90.3 \\
\hline 83 & Fenamiphos & 6.22 & 303.1 & {$[\mathrm{M}+\mathrm{H}]^{+}$} & $303.6>217.0(-23)$ & $303.6>201.9(-35)$ & 50.4 \\
\hline 84 & Fenazaquin & 9.08 & 306.2 & {$[\mathrm{M}+\mathrm{H}]^{+}$} & $306.7>161.2(-17)$ & $306.7>57.2(-26)$ & 109.4 \\
\hline 85 & Fenbuconazole & 6.06 & 336.1 & {$[\mathrm{M}+\mathrm{H}]^{+}$} & $336.9>125.0(-31)$ & $336.9>70.1(-21)$ & 77.7 \\
\hline 86 & Fenhexamid & 5.86 & 301.1 & {$[\mathrm{M}+\mathrm{H}]^{+}$} & $302.0>97.2(-24)$ & $302.0>55.1(-41)$ & 68.2 \\
\hline 87 & Fenobucarb (BPMC) & 5.12 & 207.1 & {$[\mathrm{M}+\mathrm{H}]^{+}$} & $207.9>95.0(-16)$ & $207.9>77.0(-39)$ & 30.0 \\
\hline 88 & Fenothiocarb & 6.35 & 253.1 & {$[\mathrm{M}+\mathrm{H}]^{+}$} & $253.7>72.1(-23)$ & $253.7>160.0(-10)$ & 18.3 \\
\hline 89 & Fenoxanil & 6.24 & 328.1 & {$[\mathrm{M}+\mathrm{H}]^{+}$} & $329.0>302.0(-12)$ & $329.0>86.1(-23)$ & 62.7 \\
\hline 90 & Fenoxycarb & 6.29 & 301.1 & {$[\mathrm{M}+\mathrm{H}]^{+}$} & $302.1>88.1(-21)$ & $302.1>116.1(-12)$ & 58.3 \\
\hline 91 & Fenthion & 6.61 & 278.0 & {$[\mathrm{M}+\mathrm{H}]^{+}$} & $279.0>246.9(-13)$ & $279.0>169.0(-18)$ & 118.1 \\
\hline 92 & Ferimzone & 4.73 & 254.2 & {$[\mathrm{M}+\mathrm{H}]^{+}$} & $254.6>91.1(-32)$ & $254.6>132.1(-20)$ & 118.8 \\
\hline 93 & Fipronil & 6.22 & 435.9 & {$[\mathrm{M}-\mathrm{H}]^{-}$} & $434.6>330.0(16)$ & $434.6>250.0(26)$ & 37.9 \\
\hline 94 & Fluacrypyrim & 7.24 & 426.1 & {$[\mathrm{M}+\mathrm{H}]^{+}$} & $426.9>145.0(-26)$ & $426.9>205.0(-11)$ & 40.6 \\
\hline 95 & Fluazinam & 7.97 & 464.0 & {$[\mathrm{M}-\mathrm{H}]^{-}$} & $462.7>416.0(18)$ & $462.7>398.0(16)$ & 44.1 \\
\hline 96 & Flucetosulfuron & 4.94 & 487.1 & {$[\mathrm{M}+\mathrm{H}]^{+}$} & $487.8>156.0(-20)$ & $487.8>273.0(-26)$ & 44.6 \\
\hline 97 & Flufenacet & 5.96 & 363.1 & {$[\mathrm{M}+\mathrm{H}]^{+}$} & $363.6>152.1(-20)$ & $363.6>194.1(-11)$ & 46.7 \\
\hline 98 & Flufenoxuron & 8.40 & 488.0 & {$[\mathrm{M}+\mathrm{H}]^{+}$} & $488.8>158.0(-20)$ & $488.8>141.0(-46)$ & 92.7 \\
\hline 99 & Fluopicolide & 5.54 & 382.0 & {$[\mathrm{M}+\mathrm{H}]^{+}$} & $382.5>172.9(-23)$ & $382.5>145.0(-48)$ & 58.3 \\
\hline 100 & Fluopyram & 5.81 & 396.0 & {$[\mathrm{M}+\mathrm{H}]^{+}$} & $396.5>145.1(-53)$ & $396.5>173.0(-28)$ & 88.3 \\
\hline 101 & Flusilazole & 6.21 & 315.1 & {$[\mathrm{M}+\mathrm{H}]^{+}$} & $315.6>247.0(-18)$ & $315.6>165.0(-26)$ & 101.8 \\
\hline 102 & Flusulfamide & 6.84 & 413.9 & {$[\mathrm{M}-\mathrm{H}]^{-}$} & $412.6>171.1$ & $412.6>349.0$ & 35.7 \\
\hline 103 & Flutolanil & 5.45 & 323.1 & {$[\mathrm{M}+\mathrm{H}]^{+}$} & $323.6>242.0(-26)$ & $323.6>262.0(-19)$ & 84.5 \\
\hline 104 & Fluxapyroxad & 5.47 & 381.1 & {$[\mathrm{M}+\mathrm{H}]^{+}$} & $381.5>362.0(-15)$ & $381.5>342.0(-21)$ & 95.1 \\
\hline 105 & Fonofos & 6.70 & 246.0 & {$[\mathrm{M}+\mathrm{H}]^{+}$} & $247.0>109.0(-19)$ & $247.0>137.0(-12)$ & 47.5 \\
\hline 106 & Forchlorfenuron & 4.62 & 247.1 & {$[\mathrm{M}-\mathrm{H}]^{-}$} & $245.9>127.1(11)$ & $245.9>91.0(26)$ & 12.6 \\
\hline 107 & Fosthiazate & 4.30 & 283.0 & {$[\mathrm{M}+\mathrm{H}]^{+}$} & $283.5>104.0(-24)$ & $283.5>227.9(-10)$ & 51.4 \\
\hline
\end{tabular}


Table 3. Cont

\begin{tabular}{|c|c|c|c|c|c|c|c|}
\hline \multirow{2}{*}{ No. } & \multirow{2}{*}{ Compound Name } & \multirow{2}{*}{$t_{R}(\min )$} & \multirow{2}{*}{$\begin{array}{l}\text { Mono-Isotopic } \\
\text { Mass }\end{array}$} & \multirow{2}{*}{$\begin{array}{c}\text { Ionization } \\
\text { Form of } \\
\text { Precursor Ion }\end{array}$} & Precursor Ion $>$ & uct Ion $\left(\mathrm{CE}^{1}, \mathrm{~V}\right)$ & Bation (o) \\
\hline & & & & & Quantifier & Qualifier & Katio- (\%) \\
\hline 108 & Furathiocarb & 7.76 & 382.2 & {$[\mathrm{M}+\mathrm{H}]^{+}$} & $382.6>195.0(-19)$ & $382.6>252.0(-13)$ & 46.7 \\
\hline 109 & Halosulfuron-methyl & 5.82 & 434.0 & {$[\mathrm{M}+\mathrm{H}]^{+}$} & $434.8>182.0(-22)$ & $434.8>139.0(-43)$ & 34.8 \\
\hline 110 & Haloxyfop-R-Methyl & 7.25 & 375.0 & {$[\mathrm{M}+\mathrm{H}]^{+}$} & $375.6>316.0(-18)$ & $375.6>91.1(-32)$ & 56.2 \\
\hline 111 & Hexaconazole & 6.81 & 313.1 & {$[\mathrm{M}+\mathrm{H}]^{+}$} & $313.9>70.1(-21)$ & $313.9>158.9(-32)$ & 25.3 \\
\hline 112 & Hexaflumuron & 7.38 & 460.0 & {$[\mathrm{M}-\mathrm{H}]^{-}$} & $458.8>438.9(11)$ & $458.8>175.1(34)$ & 14.6 \\
\hline 113 & Hexazinone & 4.00 & 252.2 & {$[\mathrm{M}+\mathrm{H}]^{+}$} & $252.7>170.8(-20)$ & $252.7>71.1(-32)$ & 56.7 \\
\hline 114 & Imazalil & 4.26 & 296.0 & {$[\mathrm{M}+\mathrm{H}]^{+}$} & $296.6>158.9(-23)$ & $296.6>200.9(-18)$ & 50.8 \\
\hline 115 & Imazapic & 3.41 & 275.1 & {$[\mathrm{M}+\mathrm{H}]^{+}$} & $275.6>163.0(-26)$ & $275.6>216.0(-22)$ & 68.0 \\
\hline 116 & Imazaquin & 3.92 & 311.1 & {$[\mathrm{M}+\mathrm{H}]^{+}$} & $311.6>267.0(-21)$ & $311.6>199.0(-28)$ & 100.9 \\
\hline 117 & Imazethapyr & 3.71 & 289.1 & {$[\mathrm{M}+\mathrm{H}]^{+}$} & $289.6>177.1(-27)$ & $289.6>245.1(-21)$ & 123.1 \\
\hline 118 & Imibenconazole & 7.91 & 410.0 & {$[\mathrm{M}+\mathrm{H}]^{+}$} & $410.7>125.0(-30)$ & $410.7>171.0(-20)$ & 16.0 \\
\hline 119 & Imicyafos & 3.67 & 304.1 & {$[\mathrm{M}+\mathrm{H}]^{+}$} & $304.5>201.0(-22)$ & $304.5>235.0(-18)$ & 16.1 \\
\hline 120 & Imidacloprid & 3.15 & 255.1 & {$[\mathrm{M}+\mathrm{H}]^{+}$} & $255.8>209.0(-16)$ & $255.8>175.1(-20)$ & 93.0 \\
\hline 121 & Indoxacarb & 7.26 & 527.1 & {$[\mathrm{M}+\mathrm{H}]^{+}$} & $527.9>203.0(-40)$ & $527.9>150.0(-24)$ & 65.9 \\
\hline 122 & Iprobenfos & 6.43 & 288.1 & {$[\mathrm{M}+\mathrm{H}]^{+}$} & $288.6>91.1(-29)$ & $288.6>205.0(-11)$ & 34.9 \\
\hline 123 & Iprovalicarb & 5.88 & 320.2 & {$[\mathrm{M}+\mathrm{H}]^{+}$} & $320.8>119.0(-20)$ & $320.8>203.1(-10)$ & 19.6 \\
\hline 124 & Isazofos & 5.75 & 313.0 & {$[\mathrm{M}+\mathrm{H}]^{+}$} & $313.7>162.0(-16)$ & $313.7>97.0(-34)$ & 35.6 \\
\hline 125 & Isoprocarb & 4.53 & 193.1 & {$[\mathrm{M}+\mathrm{H}]^{+}$} & $193.9>95.1(-15)$ & $193.9>77.1(-38)$ & 37.7 \\
\hline 126 & Isoprothiolane & 5.54 & 290.1 & {$[\mathrm{M}+\mathrm{H}]^{+}$} & $290.8>188.9(-22)$ & $290.8>231.0(-12)$ & 57.1 \\
\hline 127 & Isoproturon & 4.65 & 206.1 & {$[\mathrm{M}+\mathrm{H}]^{+}$} & $206.7>72.1(-21)$ & $206.7>46.1(-18)$ & 52.4 \\
\hline 128 & Isopyrazam & 7.18 & 359.2 & {$[\mathrm{M}+\mathrm{H}]^{+}$} & $359.7>244.1(-23)$ & $359.7>320.1(-21)$ & 69.9 \\
\hline 129 & Isoxathion & 6.93 & 313.1 & {$[\mathrm{M}+\mathrm{H}]^{+}$} & $313.7>105.2(-15)$ & $313.7>97.0(-35)$ & 68.7 \\
\hline 130 & Kresoxim-methyl & 6.47 & 313.1 & {$[\mathrm{M}+\mathrm{H}]^{+}$} & $314.1>222.0(-14)$ & $314.1>267.0(-8)$ & 67.3 \\
\hline 131 & Linuron & 5.22 & 248.0 & {$[\mathrm{M}+\mathrm{H}]^{+}$} & $249.0>160.0(-18)$ & $249.0>182.0(-15)$ & 68.3 \\
\hline 132 & Mandipropamid & 5.34 & 411.1 & {$[\mathrm{M}+\mathrm{H}]^{+}$} & $411.9>328.0(-16)$ & $411.9>125.0(-34)$ & 79.7 \\
\hline 133 & Mecarbam & 5.96 & 329.1 & {$[\mathrm{M}+\mathrm{H}]^{+}$} & $330.0>226.9(-9)$ & $330.0>97.0(-38)$ & 106.5 \\
\hline 134 & Mefenacet & 5.72 & 298.1 & {$[\mathrm{M}+\mathrm{H}]^{+}$} & $298.7>148.0(-14)$ & $298.7>120.1(-24)$ & 90.3 \\
\hline 135 & Mefenpyr-diethyl & 6.81 & 372.1 & {$[\mathrm{M}+\mathrm{H}]^{+}$} & $372.8>327.0(-16)$ & $372.8>160.0(-33)$ & 32.1 \\
\hline 136 & Mepronil & 5.55 & 269.1 & {$[\mathrm{M}+\mathrm{H}]^{+}$} & $269.6>119.0(-26)$ & $269.6>91.1(-41)$ & 100.4 \\
\hline 137 & Metalaxyl & 4.61 & 279.1 & {$[\mathrm{M}+\mathrm{H}]^{+}$} & $279.6>220.1(-13)$ & $279.6>192.1(-17)$ & 61.7 \\
\hline 138 & Metamifop & 7.65 & 440.1 & {$[\mathrm{M}+\mathrm{H}]^{+}$} & $440.9>288.0(-20)$ & $440.9>123.1(-28)$ & 43.0 \\
\hline 139 & Metazosulfuron & 5.14 & 475.1 & {$[\mathrm{M}+\mathrm{H}]^{+}$} & $475.9>182.0(-20)$ & $475.9>294.9(-18)$ & 49.5 \\
\hline 140 & Metconazole & 6.82 & 319.2 & {$[\mathrm{M}+\mathrm{H}]^{+}$} & $320.1>70.0(-24)$ & $320.1>125.0(-39)$ & 18.5 \\
\hline 141 & Methabenzthiazuron & 4.59 & 221.1 & {$[\mathrm{M}+\mathrm{H}]^{+}$} & $221.5>165.0(-17)$ & $221.5>150.0(-31)$ & 43.5 \\
\hline 142 & Methidathion & 4.84 & 302.0 & {$[\mathrm{M}+\mathrm{H}]^{+}$} & $302.9>145.0(-10)$ & $302.9>85.1(-22)$ & 128.1 \\
\hline 143 & Methiocarb & 5.28 & 225.1 & {$[\mathrm{M}+\mathrm{H}]^{+}$} & $225.8>121.1(-20)$ & $225.8>169.0(-10)$ & 63.1 \\
\hline 144 & Methomyl & 3.02 & 162.0 & {$[\mathrm{M}+\mathrm{H}]^{+}$} & $163.0>88.0(-10)$ & $163.0>106.1(-11)$ & 65.1 \\
\hline 145 & Methoxyfenozide & 5.57 & 368.2 & {$[\mathrm{M}+\mathrm{H}]^{+}$} & $369.0>149.0(-21)$ & $369.0>313.1(-8)$ & 18.4 \\
\hline 146 & Metobromuron & 4.51 & 258.0 & {$[\mathrm{M}+\mathrm{H}]^{+}$} & $258.5>169.9(-19)$ & $258.5>148.0(-16)$ & 59.3 \\
\hline 147 & Metolachlor & 6.16 & 283.1 & {$[\mathrm{M}+\mathrm{H}]^{+}$} & $283.6>251.9(-17)$ & $283.6>176.1(-25)$ & 63.5 \\
\hline 148 & Metolcarb & 3.80 & 165.1 & {$[\mathrm{M}+\mathrm{H}]^{+}$} & $165.9>109.1(-12)$ & $165.9>94.1(-30)$ & 29.1 \\
\hline 149 & Metominostrobin & 4.75 & 284.1 & {$[\mathrm{M}+\mathrm{H}]^{+}$} & $284.6>196.0(-18)$ & $284.6>194.0(-21)$ & 97.0 \\
\hline 150 & Metrafenone & 7.02 & 408.1 & {$[\mathrm{M}+\mathrm{H}]^{+}$} & $408.9>209.0(-15)$ & $408.9>226.9(-22)$ & 80.3 \\
\hline $151-1$ & Mevinphos_1 & 3.25 & 224.0 & {$[\mathrm{M}+\mathrm{H}]^{+}$} & $224.7>127.0(-16)$ & $224.7>193.0(-9)$ & 55.5 \\
\hline $151-2$ & Mevinphos_2 & 3.44 & 224.0 & {$[\mathrm{M}+\mathrm{H}]^{+}$} & $224.7>127.0(-16)$ & $224.7>193.0(-9)$ & 33.8 \\
\hline 152 & Milbemectin A4 & 9.49 & 542.3 & {$\left[\mathrm{M}+\mathrm{H}-\mathrm{H}_{2} \mathrm{O}\right]^{+}$} & $525.0>109.2(-27)$ & $525.0>507.2(-13)$ & 82.9 \\
\hline 153 & Molinate & 6.32 & 187.1 & {$\left[\mathrm{M}+\mathrm{NH}_{4}\right]^{+}$} & $204.6>145.1(-15)$ & $204.6>115.0(-26)$ & 52.5 \\
\hline 154 & Monocrotophos & 3.03 & 223.1 & {$[\mathrm{M}+\mathrm{H}]^{+}$} & $223.6>127.0(-15)$ & $223.6>193.0(-8)$ & 62.1 \\
\hline 155 & Myclobutanil & 5.58 & 288.1 & {$[\mathrm{M}+\mathrm{H}]^{+}$} & $289.1>70.1(-21)$ & $289.1>125.0(-32)$ & 54.5 \\
\hline 156 & Napropamide & 6.03 & 271.2 & {$[\mathrm{M}+\mathrm{H}]^{+}$} & $271.7>171.1(-19)$ & $271.7>129.1(-16)$ & 96.7 \\
\hline 157 & Nicosulfuron & 3.77 & 410.1 & {$[\mathrm{M}+\mathrm{H}]^{+}$} & $410.9>182.0(-19)$ & $410.9>213.0(-17)$ & 51.1 \\
\hline 158 & Nitenpyram & 2.94 & 270.1 & {$[\mathrm{M}+\mathrm{H}]^{+}$} & $270.6>225.0(-12)$ & $270.6>126.0(-26)$ & 106.6 \\
\hline 159 & Nuarimol & 5.16 & 314.1 & {$[\mathrm{M}+\mathrm{H}]^{+}$} & $315.0>252.0(-22)$ & $315.0>81.1(-30)$ & 50.6 \\
\hline 160 & Ofurace & 3.93 & 281.1 & {$[\mathrm{M}+\mathrm{H}]^{+}$} & $281.6>254.1(-12)$ & $281.6>160.1(-24)$ & 128.6 \\
\hline 161 & Omethoate & 2.87 & 213.0 & {$[\mathrm{M}+\mathrm{H}]^{+}$} & $213.5>125.0(-21)$ & $213.5>183.0(-11)$ & 62.6 \\
\hline 162 & Orysastrobin & 5.51 & 391.2 & {$[\mathrm{M}+\mathrm{H}]^{+}$} & $392.1>205.0(-15)$ & $392.1>116.1(-28)$ & 94.8 \\
\hline 163 & Oxadiazon & 7.99 & 344.1 & {$[\mathrm{M}+\mathrm{H}]^{+}$} & $344.9>303.0(-14)$ & $344.9>219.9(-19)$ & 99.4 \\
\hline 164 & Oxadixyl & 3.61 & 278.1 & {$[\mathrm{M}+\mathrm{H}]^{+}$} & $278.6>219.1(-12)$ & $278.6>132.1(-29)$ & 62.8 \\
\hline 165 & Oxamyl & 2.92 & 219.1 & {$\left[\mathrm{M}+\mathrm{NH}_{4}\right]^{+}$} & $236.8>90.1(-8)$ & $236.8>56.0(-45)$ & 10.7 \\
\hline 166 & Oxydemeton-methyl & 2.92 & 246.0 & {$[\mathrm{M}+\mathrm{H}]^{+}$} & $246.5>169.0(-13)$ & $246.5>109.0(-27)$ & 82.1 \\
\hline 167 & Paclobutrazol & 5.45 & 293.1 & {$[\mathrm{M}+\mathrm{H}]^{+}$} & $294.1>70.1(-21)$ & $294.1>125.1(-38)$ & 17.9 \\
\hline 168 & Pebulate & 7.17 & 203.1 & {$[\mathrm{M}+\mathrm{H}]^{+}$} & $204.1>128.1(-12)$ & $204.1>57.1(-17)$ & 97.7 \\
\hline 169 & Penconazole & 6.50 & 283.1 & {$[\mathrm{M}+\mathrm{H}]^{+}$} & $284.0>70.1(-16)$ & $284.0>159.0(-30)$ & 98.8 \\
\hline 170 & Pendimethalin & 8.30 & 281.1 & {$[\mathrm{M}+\mathrm{H}]^{+}$} & $282.1>212.0(-12)$ & $282.1>194.0(-18)$ & 15.8 \\
\hline 171 & Penoxsulam & 4.08 & 483.1 & {$[\mathrm{M}+\mathrm{H}]^{+}$} & $483.9>195.0(-28)$ & $483.9>164.0(-34)$ & 22.5 \\
\hline 172 & Penthiopyrad & 6.48 & 359.1 & {$[\mathrm{M}+\mathrm{H}]^{+}$} & $359.8>276.0(-15)$ & $359.8>177.0(-34)$ & 133.4 \\
\hline 173 & Phenmedipham & 4.81 & 300.1 & {$\left[\mathrm{M}+\mathrm{NH}_{4}\right]^{+}$} & $318.1>136.0(-24)$ & $318.1>168.0(-14)$ & 78.8 \\
\hline 174 & Phenthoate & 6.40 & 320.0 & {$[\mathrm{M}+\mathrm{H}]^{+}$} & $321.0>79.0(-43)$ & $321.0>247.0(-12)$ & 81.7 \\
\hline 175 & Phosalone & 6.92 & 367.0 & {$[\mathrm{M}+\mathrm{H}]^{+}$} & $367.7>182.0(-17)$ & $367.7>111.0(-40)$ & 68.8 \\
\hline 176 & Phosmet & 4.95 & 317.0 & {$[\mathrm{M}+\mathrm{H}]^{+}$} & $317.8>160.0(-16)$ & $317.8>77.0(-54)$ & 29.9 \\
\hline 177 & Phosphamidon & 3.70 & 299.1 & {$[\mathrm{M}+\mathrm{H}]^{+}$} & $300.0>174.0(-14)$ & $300.0>127.0(-30)$ & 60.1 \\
\hline 178 & Phoxim & 6.90 & 298.1 & {$[\mathrm{M}+\mathrm{H}]^{+}$} & $298.5>129.0(-11)$ & $298.5>77.0(-30)$ & 231.1 \\
\hline 179 & Picolinafen & 7.93 & 376.1 & {$[\mathrm{M}+\mathrm{H}]^{+}$} & $376.9>237.9(-27)$ & $376.9>358.9(-20)$ & 28.0 \\
\hline 180 & Picoxystrobin & 6.31 & 367.1 & {$[\mathrm{M}+\mathrm{H}]^{+}$} & $367.9>145.0(-21)$ & $367.9>205.1(-9)$ & 44.5 \\
\hline 181 & Piperophos & 7.29 & 353.1 & {$[\mathrm{M}+\mathrm{H}]^{+}$} & $353.7>170.9(-23)$ & $353.7>255.0(-14)$ & 50.6 \\
\hline 182 & Pirimicarb & 4.05 & 238.1 & {$[\mathrm{M}+\mathrm{H}]^{+}$} & $238.8>72.1(-23)$ & $238.8>182.1(-16)$ & 39.3 \\
\hline 183 & Pirimiphos-ethyl & 7.94 & 333.1 & {$[\mathrm{M}+\mathrm{H}]^{+}$} & $333.6>198.1(-23)$ & $333.6>182.1(-23)$ & 44.4 \\
\hline 184 & Pirimiphos-methyl & 6.97 & 305.1 & {$[\mathrm{M}+\mathrm{H}]^{+}$} & $305.7>108.0(-31)$ & $305.7>164.1(-22)$ & 90.1 \\
\hline 185 & Pretilachlor & 7.49 & 311.2 & {$[\mathrm{M}+\mathrm{H}]^{+}$} & $312.1>252.0(-17)$ & $312.1>176.1(-29)$ & 29.6 \\
\hline
\end{tabular}


Table 3. Cont.

\begin{tabular}{|c|c|c|c|c|c|c|c|}
\hline \multirow{2}{*}{ No. } & \multirow{2}{*}{ Compound Name } & \multirow{2}{*}{$t_{R}(\min )$} & \multirow{2}{*}{$\begin{array}{l}\text { Mono-Isotopic } \\
\text { Mass }\end{array}$} & \multirow{2}{*}{$\begin{array}{l}\text { Ionization } \\
\text { Form of } \\
\text { Precursor Ion }\end{array}$} & \multicolumn{2}{|c|}{ Precursor Ion > Product Ion $\left(\mathrm{CE}^{1}, \mathrm{~V}\right)$} & Ratio ${ }^{2}$ (o) \\
\hline & & & & & Quantifier & Qualifier & Katio (7) \\
\hline 186 & Probenazole & 3.76 & 223.0 & {$[\mathrm{M}+\mathrm{H}]^{+}$} & $224.0>41.1(-22)$ & $224.0>39.1(-45)$ & 44.2 \\
\hline 187 & Prochloraz & 6.81 & 375.0 & {$[\mathrm{M}+\mathrm{H}]^{+}$} & $375.8>308.0(-13)$ & $375.8>70.1(-26)$ & 43.2 \\
\hline 188 & Promecarb & 5.46 & 207.1 & {$[\mathrm{M}+\mathrm{H}]^{+}$} & $208.1>109.0(-16)$ & $208.1>151.1(-10)$ & 30.4 \\
\hline 189 & Prometryn & 5.63 & 241.1 & {$[\mathrm{M}+\mathrm{H}]^{+}$} & $241.6>158.0(-23)$ & $241.6>200.1(-18)$ & 47.8 \\
\hline 190 & Propachlor & 4.65 & 211.1 & {$[\mathrm{M}+\mathrm{H}]^{+}$} & $211.7>170.0(-15)$ & $211.7>94.1(-27)$ & 61.1 \\
\hline 191 & Propazine & 5.31 & 229.1 & {$[\mathrm{M}+\mathrm{H}]^{+}$} & $229.7>146.0(-23)$ & $229.7>188.0(-17)$ & 83.2 \\
\hline 192 & Propiconazole & 6.70 & 341.1 & {$[\mathrm{M}+\mathrm{H}]^{+}$} & $342.0>158.9(-28)$ & $342.0>69.2(-21)$ & 45.3 \\
\hline 193 & Propisochlor & 6.64 & 283.1 & {$[\mathrm{M}+\mathrm{H}]^{+}$} & $283.9>224.0(-11)$ & $283.9>43.1(-25)$ & 107.7 \\
\hline 194 & Propoxur & 3.95 & 209.1 & {$[\mathrm{M}+\mathrm{H}]^{+}$} & $209.8>111.0(-14)$ & $209.8>93.0(-25)$ & 59.8 \\
\hline 195 & Prothiofos & 9.13 & 344.0 & {$[\mathrm{M}+\mathrm{H}]^{+}$} & $344.9>240.7(-20)$ & $344.9>268.8(-12)$ & 43.7 \\
\hline 196 & Pyraclofos & 6.91 & 360.0 & {$[\mathrm{M}+\mathrm{H}]^{+}$} & $360.5>256.9(-23)$ & $360.5>138.0(-40)$ & 42.2 \\
\hline 197 & Pyraclostrobin & 6.85 & 387.1 & {$[\mathrm{M}+\mathrm{H}]^{+}$} & $388.0>163.1(-25)$ & $388.0>194.1(-13)$ & 83.5 \\
\hline 198 & Pyrazolynate & 7.02 & 438.0 & {$[\mathrm{M}+\mathrm{H}]^{+}$} & $438.6>91.1(-37)$ & $438.6>172.9(-20)$ & 76.2 \\
\hline 199 & Pyrazophos & 7.00 & 373.1 & {$[\mathrm{M}+\mathrm{H}]^{+}$} & $373.5>222.0(-21)$ & $373.5>194.0(-32)$ & 143.2 \\
\hline 200 & Pyrazoxyfen & 6.62 & 402.1 & {$[\mathrm{M}+\mathrm{H}]^{+}$} & 402.9 > $91.1(-40)$ & $402.9>105.1(-21)$ & 64.6 \\
\hline 201 & Pyribenzoxim & 7.87 & 609.2 & {$[\mathrm{M}+\mathrm{Na}]^{+}$} & $631.8>488.1(-21)$ & $631.8>180.1(-40)$ & 44.6 \\
\hline 202 & Pyributicarb & 8.13 & 330.1 & {$[\mathrm{M}+\mathrm{H}]^{+}$} & $330.6>181.0(-16)$ & $330.6>108.1(-28)$ & 108.6 \\
\hline 203 & Pyridaben & 9.00 & 364.1 & {$[\mathrm{M}+\mathrm{H}]^{+}$} & $364.6>147.1(-25)$ & $364.6>309.0(-14)$ & 75.7 \\
\hline 204 & Pyridalyl & 10.07 & 489.0 & {$[\mathrm{M}+\mathrm{H}]^{+}$} & $489.8>183.0(-18)$ & $489.8>109.0(-28)$ & 310.6 \\
\hline 205 & Pyridaphenthion & 5.65 & 340.1 & {$[\mathrm{M}+\mathrm{H}]^{+}$} & $340.5>189.0(-21)$ & $340.5>205.0(-22)$ & 57.7 \\
\hline 206 & Pyridate & 9.36 & 378.1 & {$[\mathrm{M}+\mathrm{H}]^{+}$} & $378.8>207.0(-21)$ & $378.8>351.1(-10)$ & 19.9 \\
\hline 207-1 & Pyrifenox_1 & 5.65 & 294.0 & {$[\mathrm{M}+\mathrm{H}]^{+}$} & $294.5>93.1(-22)$ & $294.5>67.1(-55)$ & 6.2 \\
\hline $207-2$ & Pyrifenox_2 & 5.94 & 294.0 & {$[\mathrm{M}+\mathrm{H}]^{+}$} & $294.5>93.1(-22)$ & $294.5>67.1(-55)$ & 7.7 \\
\hline 208 & Pyriminobac-methyl E & 5.40 & 361.1 & {$[\mathrm{M}+\mathrm{H}]^{+}$} & $361.6>330.0(-14)$ & $361.6>284.0(-30)$ & 25.6 \\
\hline 209 & Pyriminobac-methyl Z & 4.98 & 361.1 & {$[\mathrm{M}+\mathrm{H}]^{+}$} & $361.6>330.0(-15)$ & $361.6>244.0(-26)$ & 10.7 \\
\hline 210 & Pyrimisulfan & 4.69 & 419.1 & {$[\mathrm{M}+\mathrm{H}]^{+}$} & $419.5>370.0(-19)$ & $419.5>255.0(-28)$ & 87.9 \\
\hline 211 & Pyriproxyfen & 8.11 & 321.1 & {$[\mathrm{M}+\mathrm{H}]^{+}$} & $321.6>96.1(-16)$ & $321.6>78.0(-53)$ & 49.1 \\
\hline 212 & Pyroquilon & 3.93 & 173.1 & {$[\mathrm{M}+\mathrm{H}]^{+}$} & $173.8>117.1(-31)$ & $173.8>132.1(-22)$ & 88.0 \\
\hline 213 & Quinoclamine & 3.89 & 207.0 & {$[\mathrm{M}+\mathrm{H}]^{+}$} & $208.0>89.0(-39)$ & $208.0>77.0(-38)$ & 110.3 \\
\hline 214 & Rimsulfuron & 4.05 & 431.1 & {$[\mathrm{M}+\mathrm{H}]^{+}$} & $431.5>182.0(-22)$ & $431.5>325.0(-16)$ & 51.5 \\
\hline 215 & Saflufenacil & 4.90 & 500.0 & {$[\mathrm{M}+\mathrm{H}]^{+}$} & $500.8>197.9(-45)$ & $500.8>348.9(-29)$ & 88.1 \\
\hline 216 & Sethoxydim & 7.87 & 327.2 & {$[\mathrm{M}+\mathrm{H}]^{+}$} & $327.6>178.0(-20)$ & $327.6>282.1(-12)$ & 45.6 \\
\hline 217 & Simazine & 4.08 & 201.1 & {$[\mathrm{M}+\mathrm{H}]^{+}$} & $201.9>104.0(-26)$ & $201.9>124.1(-20)$ & 70.2 \\
\hline 218 & Simeconazole & 5.93 & 293.1 & {$[\mathrm{M}+\mathrm{H}]^{+}$} & $294.1>70.1(-21)$ & $294.1>135.0(-21)$ & 24.5 \\
\hline 219 & Simetryn & 4.31 & 213.1 & {$[\mathrm{M}+\mathrm{H}]^{+}$} & $213.6>68.0(-36)$ & $213.6>124.1(-20)$ & 49.3 \\
\hline 220 & Spinetoram (XDE-175-J) & 7.30 & 747.5 & {$[\mathrm{M}+\mathrm{H}]^{+}$} & $748.1>142.1(-32)$ & $748.1>98.1(-55)$ & 16.0 \\
\hline 221 & Spinosyn A & 6.79 & 731.5 & {$[\mathrm{M}+\mathrm{H}]^{+}$} & $732.0>142.1(-30)$ & $732.0>98.1(-54)$ & 17.5 \\
\hline 222 & Spinosyn D & 7.23 & 745.5 & {$[\mathrm{M}+\mathrm{H}]^{+}$} & $746.3>142.1(-31)$ & $746.3>98.1(-55)$ & 21.1 \\
\hline 223 & Spirodiclofen & 8.69 & 410.1 & {$[\mathrm{M}+\mathrm{H}]^{+}$} & $410.9>71.1(-21)$ & $410.9>313.0(-13)$ & 74.5 \\
\hline 224 & Sulfoxaflor & 3.32 & 277.0 & {$[\mathrm{M}+\mathrm{H}]^{+}$} & $278.0>174.0(-12)$ & $278.0>154.0(-29)$ & 76.4 \\
\hline 225 & Sulprofos & 8.30 & 322.0 & {$[\mathrm{M}+\mathrm{H}]^{+}$} & $322.5>218.9(-16)$ & $322.5>155.0(-24)$ & 62.3 \\
\hline 226 & ТСМТВ & 5.24 & 238.0 & {$[\mathrm{M}+\mathrm{H}]^{+}$} & $238.8>180.0(-12)$ & $238.8>136.1(-26)$ & 70.8 \\
\hline 227 & Tebuconazole & 6.53 & 307.1 & {$[\mathrm{M}+\mathrm{H}]^{+}$} & $308.1>70.1(-22)$ & $308.1>125.1(-40)$ & 14.5 \\
\hline 228 & Tebufenozide & 6.32 & 352.2 & {$[\mathrm{M}+\mathrm{H}]^{+}$} & $353.2>133.1(-20)$ & $353.2>297.1(-10)$ & 22.0 \\
\hline 229 & Tebupirimfos & 7.96 & 318.1 & {$[\mathrm{M}+\mathrm{H}]^{+}$} & $318.6>277.0(-15)$ & $318.6>153.1(-30)$ & 117.9 \\
\hline 230 & Terbuthylazine & 5.44 & 229.1 & {$[\mathrm{M}+\mathrm{H}]^{+}$} & $229.7>174.0(-17)$ & $229.7>96.1(-26)$ & 21.2 \\
\hline 231 & Terbutryn & 5.73 & 241.1 & {$[\mathrm{M}+\mathrm{H}]^{+}$} & $241.6>186.1(-19)$ & $241.6>91.0(-27)$ & 15.4 \\
\hline 232 & Tetrachlorvinphos & 6.33 & 365.9 & {$[\mathrm{M}+\mathrm{H}]^{+}$} & $366.7>127.0(-14)$ & $366.7>205.9(-38)$ & 68.7 \\
\hline 233 & Tetraconazole & 5.91 & 371.0 & {$[\mathrm{M}+\mathrm{H}]^{+}$} & $371.8>159.0(-30)$ & $371.8>70.1(-23)$ & 41.2 \\
\hline 234 & Thenylchlor & 5.96 & 323.1 & {$[\mathrm{M}+\mathrm{H}]^{+}$} & $323.8>127.0(-15)$ & $323.8>53.0(-55)$ & 20.4 \\
\hline 235 & Thiabendazole & 3.30 & 201.0 & {$[\mathrm{M}+\mathrm{H}]^{+}$} & $201.5>175.0(-24)$ & $201.5>131.1(-31)$ & 128.1 \\
\hline 236 & Thiacloprid & 3.38 & 252.0 & {$[\mathrm{M}+\mathrm{H}]^{+}$} & $252.6>126.0(-21)$ & $252.6>99.0(-43)$ & 20.6 \\
\hline 237 & Thiazopyr & 6.55 & 396.1 & {$[\mathrm{M}+\mathrm{H}]^{+}$} & $396.6>377.0(-23)$ & $396.6>334.9(-29)$ & 55.2 \\
\hline 238 & Thidiazuron & 3.91 & 220.0 & {$[\mathrm{M}-\mathrm{H}]^{-}$} & $218.9>100.0(9)$ & $218.9>71.0(32)$ & 37.2 \\
\hline 239 & Thifensulfuron-methyl & 3.75 & 387.0 & {$[\mathrm{M}+\mathrm{H}]^{+}$} & $387.8>167.1(-17)$ & $387.8>204.9(-27)$ & 22.4 \\
\hline 240 & Thiobencarb & 7.04 & 257.1 & {$[\mathrm{M}+\mathrm{H}]^{+}$} & $257.8>125.0(-20)$ & $257.8>89.0(-48)$ & 21.4 \\
\hline 241 & Thiodicarb & 4.20 & 354.0 & {$[\mathrm{M}+\mathrm{H}]^{+}$} & $355.0>88.0(-21)$ & $355.0>108.0(-15)$ & 49.3 \\
\hline 242 & Thiophanate-methyl & 3.82 & 342.1 & {$[\mathrm{M}+\mathrm{H}]^{+}$} & $342.8>151.0(-20)$ & $342.8>310.9(-11)$ & 14.5 \\
\hline 243 & Tolfenpyrad & 7.93 & 383.1 & {$[\mathrm{M}+\mathrm{H}]^{+}$} & $383.7>197.0(-25)$ & $383.7>154.1(-42)$ & 40.7 \\
\hline 244 & Triadimefon & 5.60 & 293.1 & {$[\mathrm{M}+\mathrm{H}]^{+}$} & $294.0>69.1(-22)$ & $294.0>197.0(-16)$ & 66.3 \\
\hline 245 & Triadimenol & 5.74 & 295.1 & {$[\mathrm{M}+\mathrm{H}]^{+}$} & $296.0>70.0(-12)$ & $296.0>99.0(-16)$ & 9.4 \\
\hline 246 & Tri-allate & 8.31 & 303.0 & {$[\mathrm{M}+\mathrm{H}]^{+}$} & $303.7>86.1(-17)$ & $303.7>142.9(-27)$ & 94.5 \\
\hline 247 & Triazophos & 5.75 & 313.1 & {$[\mathrm{M}+\mathrm{H}]^{+}$} & $313.5>162.0(-19)$ & $313.5>97.0(-36)$ & 34.0 \\
\hline 248 & Tribenuron-methyl & 4.44 & 395.1 & {$[\mathrm{M}+\mathrm{H}]^{+}$} & $395.8>155.0(-15)$ & $395.8>181.0(-21)$ & 64.7 \\
\hline 249 & Tribufos & 9.02 & 314.1 & {$[\mathrm{M}+\mathrm{H}]^{+}$} & $314.9>169.0(-17)$ & $314.9>112.9(-23)$ & 35.9 \\
\hline 250 & Trichlorfon & 3.31 & 255.9 & {$[\mathrm{M}+\mathrm{H}]^{+}$} & $256.9>109.1(-18)$ & $256.9>221.0(-11)$ & 30.1 \\
\hline 251 & Tricyclazole & 3.56 & 189.0 & {$[\mathrm{M}+\mathrm{H}]^{+}$} & $189.5>163.0(-22)$ & $189.5>136.0(-27)$ & 98.4 \\
\hline 252 & Trifloxystrobin & 7.34 & 408.1 & {$[\mathrm{M}+\mathrm{H}]^{+}$} & $408.6>186.0(-19)$ & $408.6>145.0(-43)$ & 97.4 \\
\hline 253 & Triflumizole & 7.40 & 345.1 & {$[\mathrm{M}+\mathrm{H}]^{+}$} & $346.0>277.9(-11)$ & $346.0>43.1(-28)$ & 70.9 \\
\hline 254 & Triflumuron & 6.86 & 358.0 & {$[\mathrm{M}+\mathrm{H}]^{+}$} & $358.8>156.0(-18)$ & $358.8>139.0(-30)$ & 76.5 \\
\hline 255 & Trimethacarb & 4.71 & 193.2 & {$[\mathrm{M}+\mathrm{H}]^{+}$} & $193.7>137.1(-12)$ & $193.7>107.0(-36)$ & 57.5 \\
\hline 256 & Triticonazole & 5.90 & 317.1 & {$[\mathrm{M}+\mathrm{H}]^{+}$} & $318.1>70.0(-22)$ & $318.1>125.0(-37)$ & 9.5 \\
\hline $257-1$ & Uniconazole_1 & 5.55 & 291.1 & {$[\mathrm{M}+\mathrm{H}]^{+}$} & $291.9>70.1(-24)$ & $291.9>125.0(-32)$ & 65.5 \\
\hline $257-2$ & Uniconazole_2 & 5.78 & 291.1 & {$[\mathrm{M}+\mathrm{H}]^{+}$} & $291.9>70.1(-24)$ & $291.9>125.0(-32)$ & 72.6 \\
\hline 258 & Vamidothion & 3.21 & 287.0 & {$[\mathrm{M}+\mathrm{H}]^{+}$} & $287.7>145.8(-18)$ & $287.7>118.0(-23)$ & 92.9 \\
\hline 259 & XMC & 4.34 & 179.1 & {$[\mathrm{M}+\mathrm{H}]^{+}$} & $180.1>123.1(-12)$ & $180.1>108.1(-27)$ & 35.0 \\
\hline 260 & Zoxamide & 6.73 & 335.0 & {$[\mathrm{M}+\mathrm{H}]^{+}$} & $335.8>186.9(-22)$ & $335.8>159.0(-40)$ & 75.9 \\
\hline
\end{tabular}



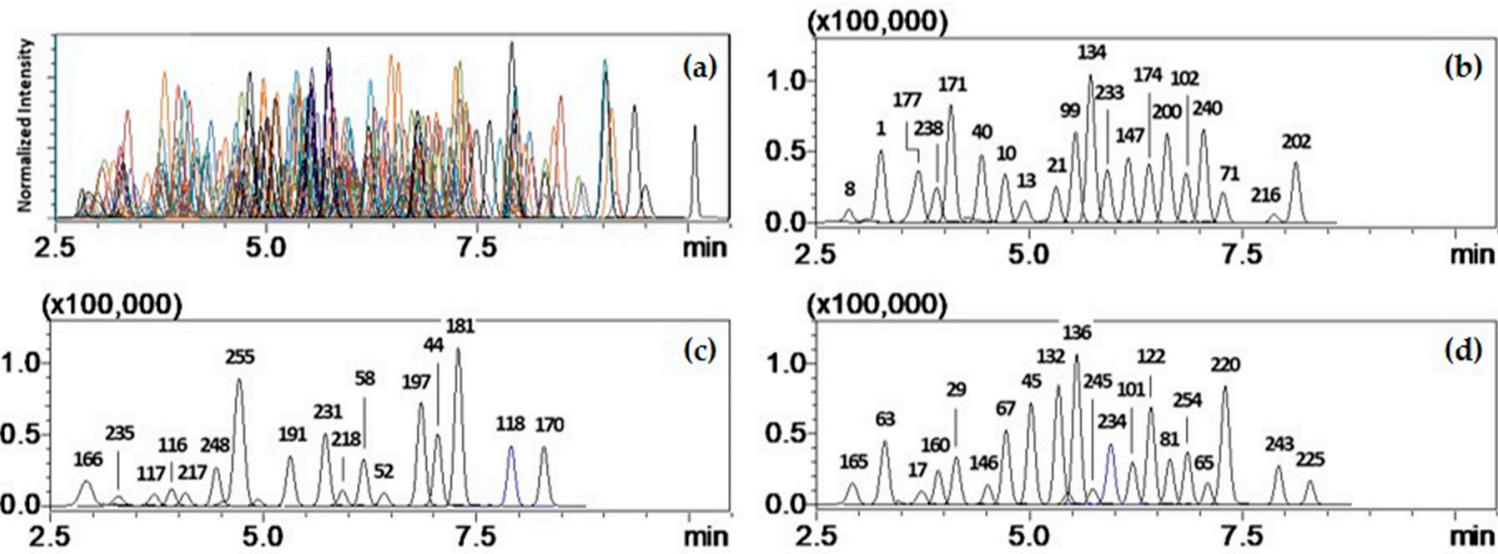

$(\times 100,000)$

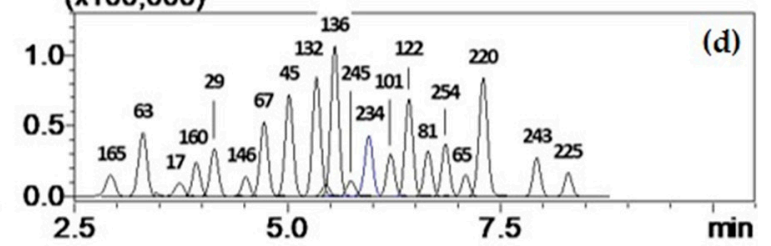

$(x 100,000)$
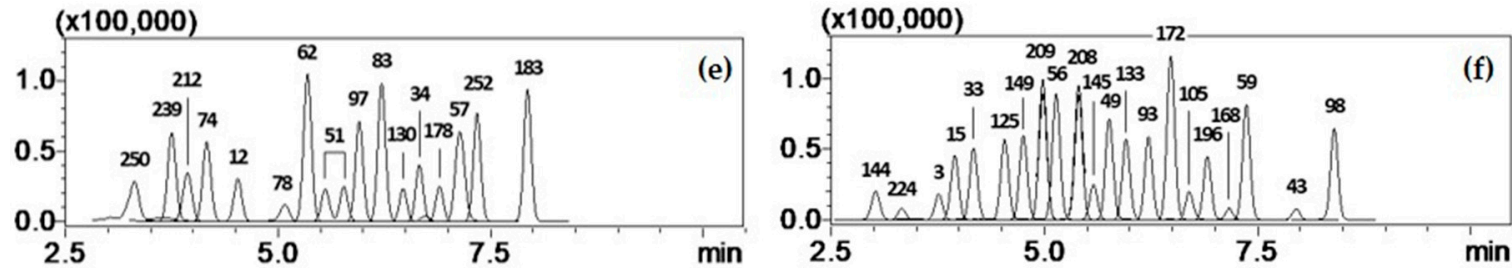

$(x 100,000)$

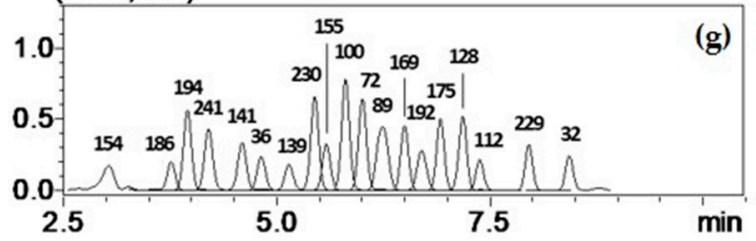

$(x 100,000)$

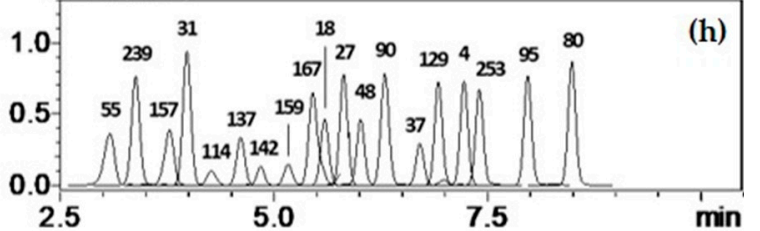

$(x 100,000)$

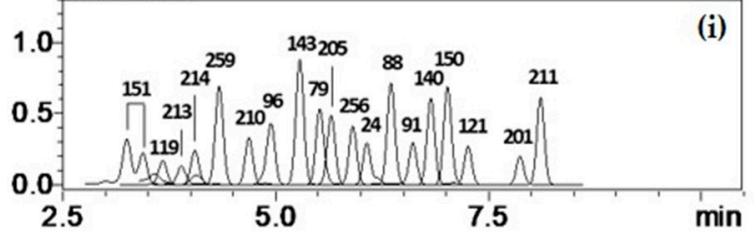

$(\mathrm{x} 100,000)$

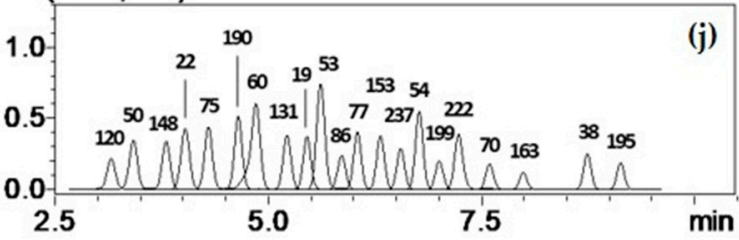

$(\times 100,000)$

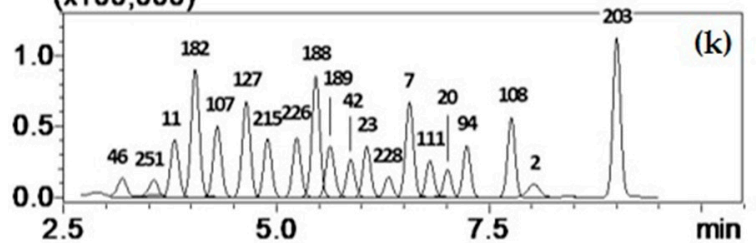

(k)

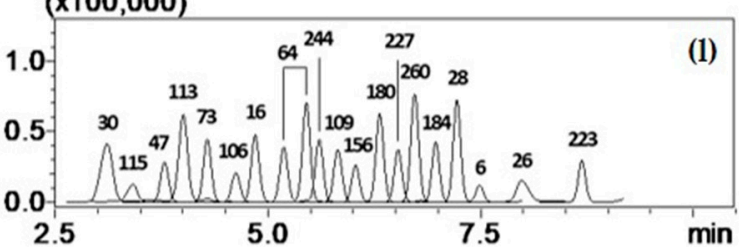

$(\times 100,000)$

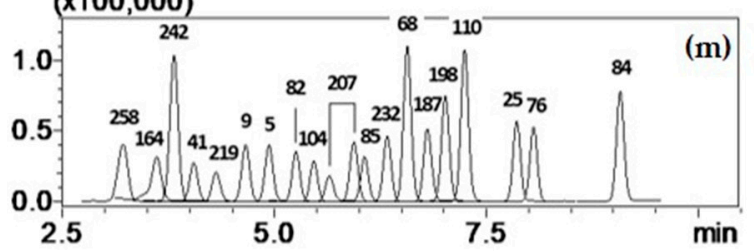

$(x 10,000)$

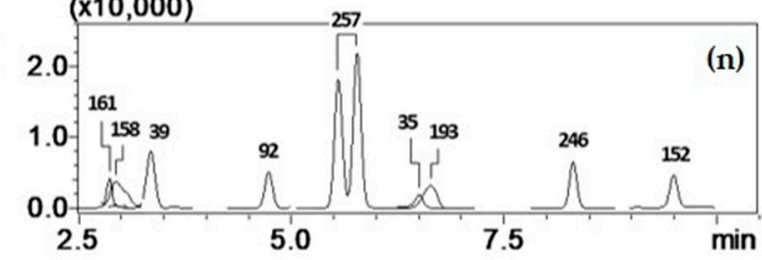

$(x 100,000)$
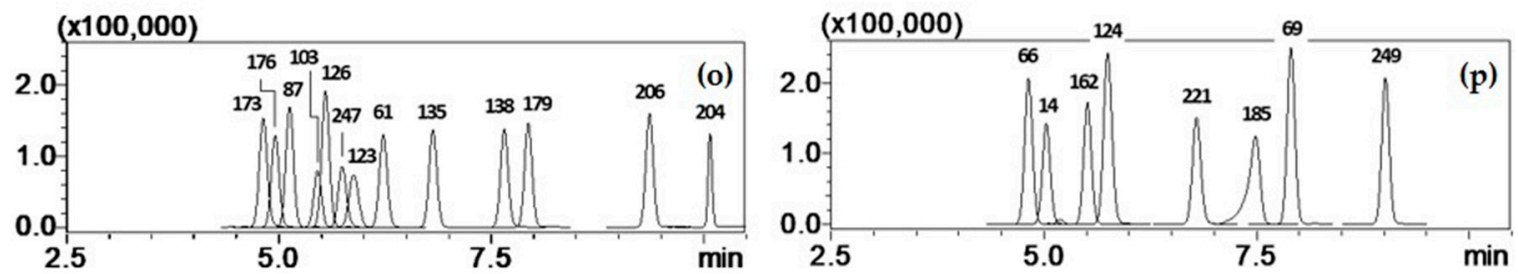

Figure 1. TIC obtained by MRM mode of LC-MS/MS at LOQ (10 ng/mL). (a) Chromatograms for 260 pesticides in urine sample ( $4 \mu \mathrm{L}$ injection); (b) to (p) Individual chromatograms separated from (a). Pesticide numbers assigned on the peaks and their MRM transitions (quantifier) are designated in Table 3. 


\subsection{Method Validation}

Analytical method validation was conducted for the 260 multiresidual pesticides in urine. The validation parameters to be determined were limit of quantitation (LOQ), linearity of calibration, accuracy/precision, recovery, and matrix effect (Table 4). 
Table 4. Linearity of calibration $\left(\mathrm{r}^{2}\right)$, accuracy and precision in intra-day and inter-day measurements, recovery, and matrix effect results for 260 pesticides.

\begin{tabular}{|c|c|c|c|c|c|c|c|c|c|c|c|c|c|c|c|c|c|c|c|c|c|c|}
\hline \multirow{3}{*}{ No. } & \multirow{3}{*}{ Compound Name } & \multirow{3}{*}{$r^{2}$} & \multicolumn{8}{|c|}{ Accuracy and Precision (Intra-Day) } & \multicolumn{8}{|c|}{ Accuracy and Precision (Inter-Day) } & \multicolumn{3}{|c|}{ Recovery, \% (RSD, \%) } & \multirow{3}{*}{$\underset{\%}{\mathrm{ME}^{1}}$} \\
\hline & & & \multicolumn{2}{|c|}{$10 \mathrm{ng} / \mathrm{mL}$} & \multicolumn{2}{|c|}{$50 \mathrm{ng} / \mathrm{mL}$} & \multicolumn{2}{|c|}{$150 \mathrm{ng} / \mathrm{mL}$} & \multicolumn{2}{|c|}{$250 \mathrm{ng} / \mathrm{mL}$} & \multicolumn{2}{|c|}{$10 \mathrm{ng} / \mathrm{mL}$} & $50 \mathrm{ng}$ & $/ \mathrm{mL}$ & $150 \mathrm{ng}$ & $\mathrm{g} / \mathrm{mL}$ & $250 \mathrm{ng}$ & $3 / \mathrm{mL}$ & & & & \\
\hline & & & $\begin{array}{l}\mathrm{RE} \\
\%\end{array}$ & $\underset{\%}{\text { RSD }}$ & $\begin{array}{l}\mathrm{RE} \\
\%\end{array}$ & $\begin{array}{c}\text { RSD } \\
\%\end{array}$ & $\begin{array}{l}\mathrm{RE} \\
\%\end{array}$ & $\begin{array}{c}\text { RSD } \\
\%\end{array}$ & $\begin{array}{l}\mathrm{RE} \\
\%\end{array}$ & $\underset{\%}{\text { RSD }}$ & $\begin{array}{l}\mathrm{RE} \\
\%\end{array}$ & $\begin{array}{c}\text { RSD } \\
\%\end{array}$ & $\begin{array}{l}\mathrm{RE} \\
\%\end{array}$ & $\underset{\%}{\text { RSD }}$ & $\begin{array}{l}\mathrm{RE} \\
\%\end{array}$ & $\underset{\%}{\text { RSD }}$ & $\begin{array}{l}\mathrm{RE} \\
\%\end{array}$ & $\underset{\%}{\text { RSD }}$ & & & & \\
\hline 1 & Acetamiprid & 1.000 & 1.5 & 5.5 & -2.8 & 2.6 & -1.2 & 5.6 & -4.1 & 1.4 & 2.0 & 10.1 & 1.2 & 4.0 & -1.2 & 2.9 & -3.6 & 7.0 & $85.4(3.8)$ & $91.7(4.3)$ & $83.9(3.6)$ & -44.0 \\
\hline 2 & (1) & 0.999 & 9.7 & 10.3 & 3.8 & 4.8 & -4.5 & 4.8 & -8.7 & 1.2 & 6.1 & 18.3 & 0.9 & 5.1 & -2.7 & 3.7 & -6.0 & 5 & $80.6(14.7)$ & $83.6(12.6)$ & $1.8(6.6)$ & 1.1 \\
\hline & Allidock & 1.000 & 17.1 & 11.9 & 10.3 & 6.6 & t. & 2.4 & -2.7 & 1.2 & 10.0 & 14.2 & 4.0 & 4.8 & -1.3 & 2.8 & & (1) & $2.5(1$ & (0) & $8)$ & -25.5 \\
\hline 4 & Ametoctradin & 0.999 & 2.1 & 10.2 & -1.3 & 2.2 & -5.5 & 2.2 & $\begin{array}{l}-2.8 \\
-6.8\end{array}$ & 2.4 & 2.7 & 14.9 & 0.9 & 4.3 & -4.1 & 5.3 & -6.0 & 5.3 & $2.6(13.7)$ & 4.1 & $1(4.8)$ & -0.8 \\
\hline 5 & Ametryn & 1.000 & 4.9 & 9.1 & 0.6 & 3.0 & -3.9 & 2.0 & -6.1 & 2.2 & -9.0 & 16.4 & -4.0 & 2.6 & -5.2 & 1.7 & -4.6 & 6.8 & $90(12.3)$ & 93.2 & $9(3.9)$ & -7.8 \\
\hline 6 & Amisulbrom & 0.997 & -8.6 & 14.6 & -0.9 & 11.6 & -4.3 & 8.4 & -4.4 & 5.2 & 2.1 & 5.5 & 5.6 & 8.0 & 1.2 & 5.7 & -3.4 & 10.1 & $110.3(7.8)$ & 97.3 (18.1) & $8(1.6)$ & -0.6 \\
\hline 7 & Anilofos & 0.999 & -0.5 & 10.6 & -0.1 & 8.0 & -7.0 & 4.1 & -9.2 & 2.9 & 13.2 & 9.4 & 0.8 & 2.3 & -3.9 & 3.9 & -8.4 & 6.3 & $73.3(6.9)$ & $97(12.6)$ & $78(4.0)$ & -3.1 \\
\hline 8 & Asulam & 0.999 & $\begin{array}{l}-0.0 \\
-11.9\end{array}$ & $\begin{array}{l}10.0 \\
16.0\end{array}$ & $\begin{array}{l}-1.1 \\
10.4\end{array}$ & $\begin{array}{l}.0 .0 \\
5.6\end{array}$ & 6.9 & $\begin{array}{l}4.1 \\
4.6\end{array}$ & 1.6 & 6.1 & 5.8 & 14.1 & 6.1 & 4.7 & 3.0 & 4.8 & $\begin{array}{l}-0.4 \\
-8.1\end{array}$ & $\begin{array}{l}0.0 \\
2.3\end{array}$ & 84.1 (15.3) & $84.2(4.1)$ & $83.3(3.4)$ & $\begin{array}{l}-5.1 \\
-51.1\end{array}$ \\
\hline 9 & Atrazin & 0.999 & -1.0 & 3.2 & 5.4 & 2.7 & 2.0 & 1.6 & -6.2 & 2.8 & -8.2 & t.t. & 0. & 5.3 & -3.4 & 5.1 & -8.6 & 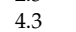 & $x_{1}$ & & & -16.5 \\
\hline 10 & Azaco & 0.997 & -10.4 & 14.2 & 7.1 & 3.4 & 2.0 & 4.6 & -7.4 & 2.4 & -8.5 & 16.0 & & 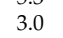 & . & & $\mathrm{cos}$ & & & & & -8.3 \\
\hline 11 & Azametl & 1.000 & $\begin{array}{l}-1.4 \\
-4.0\end{array}$ & $\begin{array}{l}8.2 \\
8.7\end{array}$ & $\begin{array}{l}1.1 \\
-0.1\end{array}$ & $\begin{array}{l}.4 \\
2.0\end{array}$ & $\begin{array}{l}-2.7 \\
-0.3\end{array}$ & $\begin{array}{l}4.0 \\
2.7\end{array}$ & -2.1 & 3.3 & $\begin{array}{l}-0.3 \\
-8.0\end{array}$ & 7.4 & $\begin{array}{l}0.5 \\
2.5\end{array}$ & 5.4 & $\begin{array}{l}-3.0 \\
-1.7\end{array}$ & 6.1 & $\begin{array}{l}-0.0 \\
-4.1\end{array}$ & 7.1 & .3) & 0) & .7) & $\begin{array}{l}-0.0 \\
-43.7\end{array}$ \\
\hline 12 & Azimsulfuron & 1.000 & -1.5 & 8.9 & -0.1 & 2.8 & -3.4 & 3.3 & -9.1 & 2.1 & -2.0 & 15.8 & -0.6 & 4.9 & -6.4 & 4.6 & -5.9 & 4.8 & $80.2(7.4)$ & 5) & 1.9) & 29.8 \\
\hline 13 & Azinphos-methyl & 0.998 & 11.1 & 11.0 & -2.4 & 8.7 & -4.0 & 5.4 & -5.7 & 2.6 & -15.0 & 11.4 & -5.5 & 5.8 & 0.3 & 4.4 & -7.9 & 6.8 & $97.1(7.9)$ & $94.3(2.5)$ & $2(1.4)$ & -7.6 \\
\hline 14 & Azoxystrobin & 1.000 & 0.9 & 3.9 & -3.6 & 3.4 & -5.2 & 2.4 & -6.5 & 2.0 & -0.8 & 5.9 & -1.6 & 3.7 & -4.3 & 1.1 & -3.7 & 5.6 & $102(5.0)$ & $90.4(4.8)$ & $6(1.7)$ & -6.1 \\
\hline $\begin{array}{l}14 \\
15\end{array}$ & xystromin & & $\begin{array}{l}0.9 \\
-8.4\end{array}$ & $\begin{array}{l}3.9 \\
8.4\end{array}$ & $\begin{array}{l}-3.0 \\
4.3\end{array}$ & $\begin{array}{l}.4 \\
6.4\end{array}$ & $\begin{array}{l}-3.2 \\
-0.2\end{array}$ & $\begin{array}{l}2.4 \\
1.5\end{array}$ & $\begin{array}{l}-0.5 \\
-5.3\end{array}$ & $\begin{array}{l}2.0 \\
1.1\end{array}$ & $\begin{array}{l}-0.0 \\
-11.9\end{array}$ & 12.3 & $\begin{array}{l}-1.0 \\
0.0\end{array}$ & $\begin{array}{l}3.7 \\
5.4\end{array}$ & $\begin{array}{c}-4.3 \\
0.9\end{array}$ & 1. & & & $\begin{array}{l}102(3.0) \\
89.7(15.9)\end{array}$ & & & $\begin{array}{l}-0.1 \\
-26.8\end{array}$ \\
\hline 16 & nethyl & & $\begin{array}{l}-9.2 \\
-9.2\end{array}$ & 8. & 0. & 3.7 & -5.9 & 6.1 & -10.3 & & -5.4 & & -0.2 & & & & & & & & & 36.1 \\
\hline 17 & Be & 0. & -16.1 & 14 & & 5 & 5.5 & 4.7 & 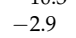 & 3.9 & 12 & 9.5 & 2 & 6.4 & - & & & & & & & -12.3 \\
\hline 18 & thiavalicarb-isopr & 0.998 & 4.1 & 11.7 & -2.5 & 5.8 & 1.9 & 7.7 & -6.5 & 3.8 & -13.0 & 13.7 & 2.1 & 6.4 & -2.3 & $\begin{array}{l}0.1 \\
5.7\end{array}$ & $\begin{array}{l}-1.0 \\
-6.5\end{array}$ & 9.1 & $\begin{array}{l}89.8(7.5) \\
89.2(17)\end{array}$ & $\begin{array}{l}\text { oo. } \\
91.7\end{array}$ & .2) & 1.4 \\
\hline 19 & Benzobicyclon & 0.994 & 1.5 & 17.2 & 7.7 & 9.3 & 6.4 & 6.1 & -9.4 & 3.2 & -7.5 & 16.5 & 14.1 & 5.4 & -1.1 & 8.7 & -6.9 & 2.4 & $84.3(8.8)$ & $85.8(1.1)$ & $6(4.3)$ & 6.4 \\
\hline 20 & & 0.999 & 3.4 & 13.4 & -1.7 & 14.7 & $\begin{array}{l}0.1 \\
-1.8\end{array}$ & 4.2 & $\begin{array}{l}-9.9 \\
-9.9\end{array}$ & 3.0 & 6.7 & 19.5 & $\begin{array}{l}-0.2 \\
-0.2\end{array}$ & 10.1 & -2.3 & 3.9 & -5.7 & 6.1 & $106.5(15.2)$ & $96.7(9.5)$ & i) & $\begin{array}{l}0.4 \\
-2.4\end{array}$ \\
\hline 21 & & 0.999 & 12.5 & 11.0 & -3.1 & 7.5 & 1.0 & 3.9 & -8.3 & 4.0 & -13.0 & 19.9 & 1.0 & 7.9 & -3.1 & 4.8 & -7.9 & 6.2 & & & & 2.6 \\
\hline $\begin{array}{l}21 \\
22\end{array}$ & & & $\begin{array}{l}1.5 \\
-5.1\end{array}$ & 11.0 & $\begin{array}{l}-3.1 \\
9.1\end{array}$ & 5.7 & 1. & 3.9 & & 4. & $\begin{array}{l}-1.0 \\
-8.1\end{array}$ & 19.9 & $\begin{array}{l}1.0 \\
7.7\end{array}$ & 7. & -3.1 & 3. & -1.9 & & & & & $\begin{array}{c}2.0 \\
-33.9\end{array}$ \\
\hline 23 & & & N.t & 10 & 0 & 4. & & & & & & 10 & & & & & & & & & & -5.2 \\
\hline 24 & & 0. & $\begin{array}{l}0.1 \\
12.4\end{array}$ & 7. & -1.8 & 4.3 .3 & -3.2 & 2 & -4.7 & 3. & -4.3 & $\begin{array}{l}10.4 \\
17.6\end{array}$ & -3.2 & 5. & 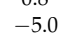 & 7. & -5.0 & 7.5 & & & & -3.0 \\
\hline 25 & & 1. & 11.6 & 8.9 & 0.0 & 7.3 & -5.1 & 2.9 & -7.8 & 2. & -5.5 & 7.3 & -0.7 & 6.0 & -2.2 & 4.6 & -5.6 & 10.1 & 75.6 & 80.8 & .2) & -7.0 \\
\hline 26 & & & -13.1 & 17.0 & -4.8 & 7.0 & -6.1 & 2.8 & -6.7 & 3. & -7.2 & 9.8 & 1.6 & 5.8 & -2.5 & 5.1 & -2.9 & 6.2 & & & .0) & -1.5 \\
\hline 27 & & & 7.4 & 9.5 & 10.3 & 2.9 & 1.0 & 27 & $-\varepsilon$ & 5.7 & -3.4 & 9.2 & 1 & 9.1 & -3 & 5. & -8.4 & 10.8 & & & & 9.0 \\
\hline 28 & & & $\begin{array}{l}1.4 \\
-10.6\end{array}$ & $\begin{array}{l}9.3 \\
10.9\end{array}$ & $\begin{array}{l}10.3 \\
1.2\end{array}$ & 10 & & & & & $\begin{array}{l}-0.4 \\
-12.4\end{array}$ & $\begin{array}{l}9.2 \\
13.6\end{array}$ & & 9. & & & - & & & & & $\begin{array}{l}.90 \\
-2.2\end{array}$ \\
\hline 28 & & & $\begin{array}{l}-10.6 \\
9.5\end{array}$ & 9. & 6. & $\begin{array}{l}1.9 \\
5.1\end{array}$ & & & & 2. & & 14 & 4 & & & 4 & & & & & & .0 \\
\hline 30 & & & -0.4 & 3. & 0. & . & & 1. & & & & & & & & & & & & & & -46.7 \\
\hline 31 & & & 8.3 & 7.5 & 4. & 2. & -1 & 2. & - & 1. & -2.7 & 9. & 2.6 & 3. & -2 & 2. & -8.3 & 6. & & & & -21.8 \\
\hline 32 & Carbs & & 3.6 & 13.6 & 1 & 5. & & 2 & & 2. & 0. & 9. & -0.9 & 4. & -2 & 2 & & & & & & -0.1 \\
\hline 33 & & & -16.4 & 10 & 2. & 5.7 & -1 & 4.2 & & 3. & -1.3 & 13. & 1. & 4.8 & -1 & 1. & -7.6 & 6.1 & & & & -24.2 \\
\hline 34 & & & -5.3 & 16.0 & 1. & 10.7 & -5 & 1.4 & -8 r & 4.2 & 8.3 & 12. & 1. & $\begin{array}{l}4.0 \\
6.6\end{array}$ & -4 & 6. & -8.4 & 5. & & 91.4 & $76.7(4.1)$ & -4.0 \\
\hline $\begin{array}{l}54 \\
35\end{array}$ & 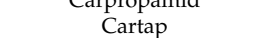 & 0 & $\begin{array}{l}-5.0 \\
-2.2\end{array}$ & $\begin{array}{l}10.0 \\
4.9\end{array}$ & 4. & $\begin{array}{l}10.7 \\
7.0\end{array}$ & -4 & 4 & $\begin{array}{l}-9.0 \\
-14.3\end{array}$ & 4 & $\begin{array}{l}0 . \\
15\end{array}$ & 10 & 0. & 0. & $\begin{array}{l}-4 \\
-0\end{array}$ & $\begin{array}{l}0 . \\
9 . \\
0.9\end{array}$ & -0.4 & 12 & 80. & 5.2 & 7) & $\begin{array}{l}-4.0 \\
-5.7\end{array}$ \\
\hline a & & & 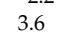 & 6. & t. & -1 & & & & & & & & & & & & & & & & \\
\hline 37 & & & -1 & 18 & 3. & & & 4 & & & & & & & -5 & & & & & & & \\
\hline 38 & & & -3 & 7. & -2 & 4. & -6 - & 10 & & 8 & -6 & 10 & $\begin{array}{l}.1 \\
-4.0\end{array}$ & & & 10 & & 12 & & & & \\
\hline 39 & & & 15 & 7. & 2. & -1 & 6 & 4 & & 4. & 9 & - & 4. & 6. & 6 & 4. & & 7.7 & & & & -56.0 \\
\hline 40 & & & ( & 4.8 & 1. & 1. & & & & 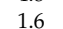 & & & -0.7 & 2. & & 4. & -5.0 & 5. & 10 & & $82(0.6)$ & -37.1 \\
\hline 41 & C & 0.996 & 11.7 & $\begin{array}{l}7.0 \\
12.0\end{array}$ & 10.4 & 4.7 & & 2.3 & -7.8 & 2.7 & -9.8 & 9.8 & 6.0 & 2. & -6.7 & 3. & -6.5 & 7. & $55.2(12.7)$ & $95.3(8.3)$ & $81.1(2.0)$ & 39.8 \\
\hline $\begin{array}{l}41 \\
42\end{array}$ & & & -18.4 & $\begin{array}{l}12.0 \\
9.9\end{array}$ & $\begin{array}{l}10.4 \\
4.6\end{array}$ & 4. & & $2.7 x-3$ & & 2.7 & $\begin{array}{l}-9.8 \\
-1.6\end{array}$ & $\begin{array}{l}9.8 \\
12.0\end{array}$ & 6. & & & 3. & & 4. & & 9) & & $\begin{array}{l}39.8 \\
-7.5\end{array}$ \\
\hline 43 & & & -5 . & 13 & 3. & & & & & & & 16 & & & & & & & & & & -0.9 \\
\hline 44 & & & & 11 & 4 & 6 & & 3 & & & & 9 & & 4 & & & & & & & & \\
\hline $\begin{array}{l}44 \\
45\end{array}$ & & & -5 & 11 & 0 & 3. & & 3 & & 3. & & 13 & & $\begin{array}{l}4 \\
1 \\
\end{array}$ & & 3. & & & & & & \\
\hline 46 & & & -1.8 & 10.2 & -4.4 & 9.6 & -3 & 6. & -5 & 4.7 & 10 & 10 & -6.1 & 6. & -3.3 & 10.0 & -7.3 & 6. & & 75.8 & $9)$ & -44.0 \\
\hline 47 & Cyanazine & 0.999 & -6.3 & 5.1 & 0.1 & 6.8 & -2.6 & 2.3 & -4.9 & 1.3 & -2.9 & 7.5 & 0.0 & 4.6 & -4.8 & 2.2 & -5.9 & 3.7 & $100(0.5)$ & $92.9(2.5)$ & $79.8(5.3)$ & -23.4 \\
\hline 48 & Cyazofamid & 0.992 & -16.7 & $\begin{array}{l}0.1 \\
16.9\end{array}$ & 9.8 & $\begin{array}{l}.0 \\
6.3\end{array}$ & -0.5 & 5.7 & -10.9 & 1.7 & -0.1 & 9.2 & 10.5 & $\begin{array}{l}4.0 \\
5.3\end{array}$ & -3.7 & 3.0 & -11.3 & 2.6 & $83.7(2.2)$ & $100.9(1.8)$ & $76.2(6.1)$ & 3.5 \\
\hline
\end{tabular}


Table 4. Cont.

\begin{tabular}{|c|c|c|c|c|c|c|c|c|c|c|c|c|c|c|c|c|c|c|c|c|c|c|}
\hline \multirow{3}{*}{ No. } & \multirow{3}{*}{ Compound Name } & \multirow{3}{*}{$r^{2}$} & \multicolumn{8}{|c|}{ Accuracy and Precision (Intra-Day) } & \multicolumn{8}{|c|}{ Accuracy and Precision (Inter-Day) } & \multicolumn{3}{|c|}{ Recovery, \% (RSD, \%) } & \multirow{3}{*}{$\underset{\%}{\mathrm{ME}^{1}}$} \\
\hline & & & \multicolumn{2}{|c|}{$10 \mathrm{ng} / \mathrm{mL}$} & \multicolumn{2}{|c|}{$50 \mathrm{ng} / \mathrm{mL}$} & \multicolumn{2}{|c|}{$150 \mathrm{ng} / \mathrm{mL}$} & \multicolumn{2}{|c|}{$250 \mathrm{ng} / \mathrm{mL}$} & $10 \mathrm{ng}$ & $/ \mathrm{mL}$ & $50 \mathrm{n}$ & $\mathrm{g} / \mathrm{mL}$ & $150 \mathrm{ng}$ & $\mathrm{g} / \mathrm{mL}$ & $250 \mathrm{ng}$ & $\mathrm{g} / \mathrm{mL}$ & & & 250 & \\
\hline & & & $\begin{array}{l}\mathrm{RE} \\
\%\end{array}$ & $\begin{array}{c}\text { RSD } \\
\%\end{array}$ & $\begin{array}{l}\mathrm{RE} \\
\%\end{array}$ & $\begin{array}{c}\text { RSD } \\
\%\end{array}$ & $\begin{array}{l}\mathrm{RE} \\
\%\end{array}$ & $\begin{array}{c}\text { RSD } \\
\%\end{array}$ & $\begin{array}{l}\mathrm{RE} \\
\%\end{array}$ & $\begin{array}{c}\text { RSD } \\
\%\end{array}$ & $\begin{array}{l}\mathrm{RE} \\
\%\end{array}$ & $\begin{array}{c}\text { RSD } \\
\%\end{array}$ & $\begin{array}{l}\text { RE } \\
\%\end{array}$ & $\underset{\%}{\text { RSD }}$ & $\begin{array}{l}\mathrm{RE} \\
\%\end{array}$ & $\begin{array}{c}\text { RSD } \\
\%\end{array}$ & $\begin{array}{l}\mathrm{RE} \\
\%\end{array}$ & $\begin{array}{c}\text { RSD } \\
\%\end{array}$ & & & & \\
\hline 49 & Cyclosulfamuron & 0.999 & 9.9 & 6.0 & 2.7 & 3.9 & -8.0 & 4.5 & -12.2 & 1.7 & -8.3 & 17.8 & -2.0 & 1.1 & -3.9 & 4.2 & -7.3 & 4.5 & $96.1(12.8)$ & $94.7(11.0)$ & $84(4.0)$ & 12.1 \\
\hline 50 & Cymoxanil & 1.000 & 6.7 & 8.6 & 9.8 & 8.4 & 7.8 & 3.7 & -0.1 & 2.1 & 9.4 & 19.1 & 6.9 & 4.8 & 3.8 & 5.1 & -4.8 & 7.9 & $0.5(13.9)$ & $91.5(0.9)$ & $86.9(6.5)$ & -61.7 \\
\hline 51 & Cyproconazole & 98 & -5.2 & 12.1 & 3.4 & 6.7 & -4.8 & 5.3 & -9.8 & 4.1 & -9.5 & 6.8 & 6.0 & 3.3 & -3.8 & 4.3 & -7.5 & 6.4 & $5.3(9.7)$ & $93(8.6)$ & $76(2.4)$ & 2.8 \\
\hline 52 & Cyprodinil & 99 & 7.8 & 12.5 & 3.8 & 7.9 & -3.7 & 4.6 & -3.9 & 3.1 & -1.5 & 15.6 & 2.8 & 2.7 & -4.2 & 3.4 & -3.0 & 7.0 & $7.2(18.8)$ & $96.9(13.8)$ & $85(4.8)$ & -14.3 \\
\hline 53 & Daimuron & 0.997 & 1.5 & 6.6 & -0.1 & 5.8 & -1.6 & 3.7 & -6.4 & 0.9 & -1.2 & 73 & 3.1 & 4.8 & -3.5 & 2.4 & -6.3 & 70 & $87.1(3.0)$ & $87.8(8.1)$ & $79.3(6.2)$ & -3.2 \\
\hline 54 & Diazinon & 1.000 & -2.9 & 5.0 & 2.2 & 2.8 & -3.3 & 6.1 & -4.7 & 5.5 & 3.4 & 13.6 & 0.9 & 4.2 & -4.7 & & & & & & & -7.2 \\
\hline 55 & Dicrotop & 0 & 0.7 & 8.0 & 2.0 & 2.5 & -4.3 & 1.4 & -7.2 & 2.6 & 4.0 & 13.1 & 4.5 & 4.4 & -1.2 & .2 & -7.4 & 0 & & & & -41.5 \\
\hline 56 & Diethofencarb & & -0.6 & 4.6 & -5.4 & 6.9 & -1.3 & 2.0 & -7.3 & 2. & -6.6 & 11.8 & 2.0 & 7.9 & -2.1 & 5 & -4.5 & 1 & .9) & 2) & $6(4.5)$ & -3.4 \\
\hline 57 & Difenoconazole & 00 & 1.3 & 6.8 & -0.8 & 4.9 & -4.1 & 3.6 & -5.3 & 1.2 & -2.2 & 17.9 & -4.1 & 3.4 & -7.5 & 3.5 & -4.2 & 4.7 & $94.1(4.6)$ & 90.5 & $4(1.9)$ & 2.6 \\
\hline 58 & Diflubenzuron & 0.998 & -3.3 & 19.7 & 9.2 & 14.9 & -1.6 & 4.6 & -8.5 & 3.6 & 0.4 & 9.9 & 0.1 & 14.0 & 3.9 & 2.2 & -9.4 & 9.7 & $93.5(5.5)$ & 88.6 (12.0) & $73(6.8)$ & 4.9 \\
\hline 59 & flufenican & 0.999 & 5.7 & 4.5 & -1.0 & 4.9 & -8.4 & 2.8 & -6.7 & 1.9 & 3.4 & 15.5 & -3.5 & 9.1 & -4.0 & 4.9 & -3.4 & 8.5 & $97.8(4.6)$ & $88.4(7.3)$ & 73.7 (11.6) & -0.6 \\
\hline 60 & . & & -1.6 & 15.5 & 5.0 & 3.6 & 0.2 & 2.5 & $\begin{array}{l}-6.4 \\
-6.4\end{array}$ & 2.0 & $\begin{array}{l}5.4 \\
-5.4\end{array}$ & 11.6 & 3.4 & 3.3 & -2.9 & 4.9 & $\begin{array}{l}-0.4 \\
-6.3\end{array}$ & 0.5 & & & $80.9(2.8)$ & $\begin{array}{l}-0.0 \\
-8.7\end{array}$ \\
\hline 61 & & & 9.8 & 58 & -1.1 & 4.4 & -2 & 2. & 4 & & 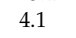 & 6 & -4.6 & & . & & & & & & & 5.8 \\
\hline 62 & $\mathrm{D}$ & 9 & 19.5 & 2.7 & 1.6 & 4.7 & -4.3 & 1.5 & -8.1 & 3.0 & 2.0 & 13.1 & 2.0 & 4.1 & -0.3 & 4 & -7.0 & 5.7 & & & & -10.1 \\
\hline 63 & Dimethoate & & -1.2 & 10.0 & 2.0 & 3.4 & 0.5 & 4.6 & -2.0 & 2.0 & -0.1 & 10.9 & 2.6 & 4.5 & 0.4 & 2.0 & -3.4 & 7.4 & & .7) & .9) & -44.2 \\
\hline 64 & Dimethomorph & 0.999 & 13.3 & 7.7 & 0.3 & 7.8 & -4.2 & 2.2 & -8.8 & 0.9 & 1.6 & 15.6 & 1.2 & 5.3 & -1.4 & 3.9 & -5.8 & 7.4 & 79.2 & $5.3(9.3)$ & 3.6) & 29.9 \\
\hline 65 & iconazole & 99 & 8.7 & 15.6 & -0.3 & 6.8 & -4.9 & 7.0 & -9.4 & 2.6 & -4.2 & 13.5 & -6.8 & 5.3 & -3.2 & 6.2 & -7.9 & 7.2 & $108.4(3.2)$ & 3.6) & $6.3(3.5)$ & -2.6 \\
\hline 66 & & 99 & -5.4 & 4.0 & 1.5 & 2.0 & -2.1 & 2.3 & -5.2 & 1. & -2.0 & 4.6 & 1.5 & 3.3 & -3.7 & 0. & -6.3 & 1.2 & & & & -9.2 \\
\hline 67 & & & -7.8 & & & & & & & & & & & & & & & & & & & 18.5 \\
\hline 68 & & & -4 & 11 & & & & & & & 9 & 16.1 & & & & & & & & & & 0.7 \\
\hline 69 & Emamectin B1a & 1.000 & 14.7 & 3.5 & -3.7 & 3.6 & -7.3 & 2 & -6.0 & 2. & 5.6 & 10.4 & -4.6 & 1.6 & -6.8 & 3.1 & -4.2 & 5.4 & & & & -0.6 \\
\hline 70 & amectin & & 9.2 & 7.4 & -1.3 & 11.0 & -14.0 & 3.9 & -12.2 & 2. & 9.4 & 9.6 & -5.7 & 13.6 & -6.3 & 9.5 & -7.5 & 5.6 & 98.5 & $87.4(19.4)$ & 7.9) & 0.1 \\
\hline 71 & EPN & & -8.6 & 4.2 & 7.5 & 2.6 & 0.8 & 2.8 & -7.5 & 1.9 & -11.4 & 9.4 & 6.9 & 0.6 & -1.8 & 1.8 & -8.3 & 3.4 & & & & -8.9 \\
\hline 72 & 2 & & 4.7 & 9.3 & 2.8 & 5.1 & -4 & 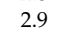 & & 2 & -3.8 & 13.4 & -0.5 & & & & & & & & & 2.9 \\
\hline 73 & & & -8.2 & 5.5 & & . & & 2. & & & & 4 & 2.4 & & & & & & & & & 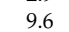 \\
\hline 74 & $\mathrm{me}$ & & 10 & 4 & & 3.5 & & & & & & & & & & & & & & & & .7 \\
\hline 75 & & & -7. & 3. & -1.0 & 3. & -2 & 1. & - & 3 & 1 & 9 & & & -2.4 & & -5.1 & & & & & -21.6 \\
\hline 76 & & & -7.6 & 8. & 1.5 & 5.1 & -3 & 1. & & 3 & -4.8 & 7 & & 4 & -2.9 & 4 & -5.8 & & & & & $\begin{array}{l}-2.0 \\
-3.4\end{array}$ \\
\hline 77 & & & -8.7 & 7.5 & -1.7 & 5.5 & - & 2. & & 2 & -4.8 & 15.1 & 0.0 & & 1. & & -5.4 & .5 & & & & -5.7 \\
\hline 78 & & & -5.0 & 7.1 & -7.3 & 4.6 & & 8 & - & 6 & -18.2 & 13.2 & -5.0 & 7. & -10.6 & 4 & 0.1 & 7 & & & & -8.4 \\
\hline 79 & & & 7.1 & 12.3 & 6.3 & 64 & & 2 & & & 0.9 & 10 & 6.9 & & & & & & & & & 8.5 \\
\hline 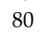 & & & 13 & 4. & & & & & & & & & & & & & & & & & & 0 \\
\hline 81 & & & to & & & & & & & & & & & & & & & & & & & 3.8 \\
\hline 82 & & & 14. & 9. & - & 3. & - & 2 & & 2 & & 10 & -0 . & 5 & & & -3.4 & 6. & & & & 3 \\
\hline 83 & & & 15 & 7. & 1 & 0. & & 1 & & 1 & & e & & 3 & & & & & & & & 0.9 \\
\hline 84 & & & 2. & 2. & & & & 2 & & 0 & & 4 & -1.9 & & & & & & & & & 3 \\
\hline 85 & & & 6. & 13 & -2.5 & 7. & & 4. & & & $-\varepsilon$ & 16.6 & 1 & & & & -4.4 & & & & & 5.0 \\
\hline 86 & & 8 & -4.3 & 19 & 5.4 & 7. & & 5. & & 2. & -3.2 & 6.0 & & & & 8. & - & & t) & & 7) & -0.6 \\
\hline 87 & nob & & 3. & 4. & 1 & 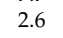 & & 3. & & & & & & & & & & & & & & -10.2 \\
\hline 88 & & & -4 & & & & & & & & & & & & & & & & & & & \\
\hline 89 & & & & 1 & & & & 1 & & & & & & & & & & & & & & \\
\hline 90 & & & -6 & & & & & 2. & & & & & & & & & & & & & & -2.1 \\
\hline 91 & & & 19.3 & 5. & 6. & 14.5 & -0 & 3. & -5 & 4.2 & -2.6 & 16.1 & & 7. & -3.5 & 4. & -6.6 & 5.4 & 96.7 & $92.5(7.5)$ & 79.6 & -5.4 \\
\hline 92 & $\mathrm{Fe}$ & & -4.5 & 8. & -0.8 & 5.2 & & 3. & & & -3.9 & 3.6 & -4.8 & 5 & & & -6.0 & & & $86.2(8.2)$ & & $\begin{array}{l}-.4 \\
-2.2\end{array}$ \\
\hline 93 & & & 14. & 7 & -1.1 & 6 & & 7. & & 1 & & 13 & -4.2 & & & & & 5 & & & & 2.5 \\
\hline 0 & & & -8 & & 2 & & & & & & 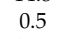 & 1 & & & & & & & & & & .7 \\
\hline 95 & & & -6 & & 3 & & & 3 & & 3 & 1 & 1 & & & & & & & & & & \\
\hline 96 & & & 0 & 9 & 1 & & & 3 & & 2. & 1 & 13 & & & & s. & & 7 & & & & 6.2 \\
\hline 97 & & 0.99 & -15.7 & 15.6 & 7. & 8.1 & -3.8 & 3. & -14.5 & 3.7 & 1.5 & 7. & 7 & 3 & -4.7 & 2. & -10.7 & 2.9 & & & & -4.7 \\
\hline 98 & Flufenoxuron & 1.000 & 8.8 & 5.2 & -1.8 & 4.4 & -6.2 & 2.5 & -4.7 & 1.4 & 4.7 & 7.8 & -3.6 & 3.4 & -4.9 & 4.3 & -4.3 & 7.6 & $95.1(8.2)$ & $86.9(6.0)$ & $77.9(5.8)$ & -2.6 \\
\hline
\end{tabular}


Table 4. Cont.

\begin{tabular}{|c|c|c|c|c|c|c|c|c|c|c|c|c|c|c|c|c|c|c|c|c|c|c|}
\hline \multirow{3}{*}{ No. } & \multirow{3}{*}{ Compound Name } & \multirow{3}{*}{$r^{2}$} & \multicolumn{8}{|c|}{ Accuracy and Precision (Intra-Day) } & \multicolumn{8}{|c|}{ Accuracy and Precision (Inter-Day) } & \multicolumn{3}{|c|}{ Recovery, \% (RSD, \%) } & \multirow{3}{*}{$\underset{\%}{\mathrm{ME}^{1}}$} \\
\hline & & & \multicolumn{2}{|c|}{$10 \mathrm{ng} / \mathrm{mL}$} & \multicolumn{2}{|c|}{$50 \mathrm{ng} / \mathrm{mL}$} & \multicolumn{2}{|c|}{$150 \mathrm{ng} / \mathrm{mL}$} & \multicolumn{2}{|c|}{$250 \mathrm{ng} / \mathrm{mL}$} & $10 \mathrm{ng}$ & $/ \mathrm{mL}$ & $50 \mathrm{n}$ & $/ \mathrm{mL}$ & $150 \mathrm{ng}$ & $\mathrm{g} / \mathrm{mL}$ & $250 \mathrm{ng}$ & $\mathrm{g} / \mathrm{mL}$ & & & & \\
\hline & & & $\begin{array}{l}\mathrm{RE} \\
\%\end{array}$ & $\underset{\%}{\text { RSD }}$ & $\begin{array}{l}\mathrm{RE} \\
\%\end{array}$ & $\underset{\%}{\text { RSD }}$ & $\begin{array}{l}\text { RE } \\
\%\end{array}$ & $\begin{array}{c}\text { RSD } \\
\%\end{array}$ & $\begin{array}{l}\mathrm{RE} \\
\%\end{array}$ & $\underset{\%}{\text { RSD }}$ & $\begin{array}{l}\mathrm{RE} \\
\%\end{array}$ & $\underset{\%}{\text { RSD }}$ & $\begin{array}{c}\mathrm{RE} \\
\%\end{array}$ & $\underset{\%}{\text { RSD }}$ & $\begin{array}{l}\mathrm{RE} \\
\%\end{array}$ & $\underset{\%}{\text { RSD }}$ & $\begin{array}{l}\mathrm{RE} \\
\%\end{array}$ & $\underset{\%}{\text { RSD }}$ & & & & \\
\hline 99 & Fluopicolide & 0.992 & -9.0 & 17.5 & 8.6 & 5.3 & -1.7 & 1.9 & -14.7 & 3.8 & 4.0 & 9.7 & 9.2 & 6.1 & 0.8 & 5.2 & -9.2 & 8.0 & $98.1(9.2)$ & $95.8(4.7)$ & $79.7(10.6)$ & -3.9 \\
\hline 100 & (1) & 0.998 & 4.5 & 9.5 & -2.2 & 4.9 & -6.6 & 5.0 & -10.3 & 1 & -8.1 & 14.5 & 2.7 & 55 & -1.6 & 7.0 & -10.2 & (2) & $82.9(12.9)$ & $8(5.4)$ & $72.1(6.2)$ & -0.6 \\
\hline 101 & Flusilaz & 00 & -5.6 & 6.1 & -2.1 & 8.5 & & 2.5 & -8.6 & 3.6 & 1.2 & 9.2 & -0.8 & 9.5 & -3.6 & 5 & -4 & & .2 & & & -1.5 \\
\hline 102 & Flusulfan & 99 & 10.0 & 11.6 & 1.8 & 5.5 & -3.2 & 4.4 & -9.6 & 3.5 & 5.7 & 7.2 & -0.7 & 8.3 & -4.5 & 6.9 & -4.8 & 2 & $3.3(2.4)$ & 6) & $\begin{array}{l}3.0(1.4) \\
83(1.6)\end{array}$ & 2.8 \\
\hline 103 & Flutolanil & 95 & -1.4 & 17.3 & 8.7 & 5.2 & -1.8 & 3.2 & -10.4 & 3.0 & -4.6 & 7.3 & 1.8 & 6.0 & -6.0 & 4.2 & -10.0 & 6.3 & $104.2(5.5)$ & $1.4(8.7)$ & $77.8(2.1)$ & -7.2 \\
\hline 104 & Fluxapyroxad & 0.988 & 1.7 & 6.8 & 11.3 & 6.8 & -1.0 & 8.2 & -9.8 & 6.3 & -10.1 & 17.3 & 14.0 & 8.4 & 1.3 & 5.5 & -4.5 & 3.7 & $54.2(11.9)$ & 90.3 (11.4) & $80(4.4)$ & -2.4 \\
\hline 105 & Fonofos & 1.000 & 9.3 & 19.6 & -6.1 & 10.9 & -6.6 & 5.7 & -7.5 & 3.9 & -0.8 & 18.3 & -2.9 & 12.3 & 1.3 & 6.7 & -3.0 & 9.5 & $86.4(3.7)$ & $82.2(14.5)$ & $77.9(4.4)$ & 8.7 \\
\hline 106 & Forchlorfenuron & 1.000 & -6.6 & 10.6 & 0.1 & 4.2 & -5.9 & 2.1 & -4.8 & 2.3 & -0.7 & 14.3 & 2.0 & 2.0 & -3.7 & 2.7 & -1.5 & 7.8 & $100.8(7.3)$ & $93.1(8.1)$ & $84.9(5.6)$ & 2.3 \\
\hline 107 & Fosthiazate & & 1.9 & 2.7 & -0.1 & 2.1 & -3.3 & 2.6 & -5.7 & 2.4 & -2.5 & Now & -1.0 & 4.8 & -3.5 & & -4.1 & & & & & -15.4 \\
\hline 108 & Furathi & 00 & -7.6 & 2.5 & -4.2 & 3.4 & -4.6 & 3.7 & -9.6 & 1. & -5.7 & 7.6 & -1.0 & 9.7 & -2.3 & 4 & -3.6 & 9.9 & & & & -4.4 \\
\hline $\begin{array}{l}100 \\
109\end{array}$ & losulfuror & 0.999 & 1.9 & $\begin{array}{l}2.0 \\
12.6\end{array}$ & $\begin{array}{l}-4.2 \\
-1.7\end{array}$ & $\begin{array}{l}3.4 \\
10.0\end{array}$ & $\begin{array}{l}-4.0 \\
-6.4\end{array}$ & $\begin{array}{l}3.0 \\
2.0\end{array}$ & $\begin{array}{l}-9.0 \\
-9.2\end{array}$ & 1. & $\begin{array}{l}-3.7 \\
-4.0\end{array}$ & 14.0 & $\begin{array}{l}-1.0 \\
-0.1\end{array}$ & 5.2 & $\begin{array}{l}-2.3 \\
-3.2\end{array}$ & 8 & $\begin{array}{l}-5.0 \\
-6.3\end{array}$ & 2 & 5) & .7) & .7) & $\begin{array}{l}-4.4 \\
13.8\end{array}$ \\
\hline 110 & loxyfop-R-Meth & 0.996 & 4.0 & 11.5 & 4.4 & 5.3 & -4.7 & 3.2 & -11.1 & 3.5 & 0.5 & 7.3 & 4.0 & 5.1 & -1.2 & 3.7 & -7.1 & 5.5 & $72.4(6.7)$ & $92(9.7)$ & 5.4) & -2.3 \\
\hline 111 & caconazole & 1.000 & 2.5 & 10.3 & 1.0 & 5.7 & -6.4 & 3.4 & -7.9 & 3.0 & -4.3 & 5.8 & -2.2 & 6.2 & -6.4 & 6.3 & -5.1 & 7.9 & $79.1(16.6)$ & $84.4(1.6)$ & $75.9(3.8)$ & -2.7 \\
\hline 112 & (1) & 0.999 & -6.1 & 13.0 & 1.9 & 11.0 & -4.6 & 2.8 & -9.0 & 3.2 & -0.1 & 17.8 & -4.5 & 10.1 & -3.0 & 3.3 & -6.5 & 4.5 & $104.1(2.4)$ & $95.9(10.4)$ & $93.8(4.1)$ & 9.4 \\
\hline 113 & & & 2.0 & 3.0 & 0.9 & 100 & -2.4 & t & -6.3 & . & 00 & 2 & 0.7 & & & & -5.4 & & & & & -23.8 \\
\hline 114 & & & 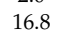 & 10 & ${ }^{0}$ & 4.0 & - & 2. & & & & 17.9 & & & & & & & & & & 22.2 \\
\hline 115 & aza & 00 & -3.7 & 6.8 & $\begin{array}{l}-1.9 \\
-1.9\end{array}$ & 4.9 & -3.0 & 2.0 & -3.6 & 4. & 7.6 & 18.4 & 1.6 & 3.8 & -3.3 & 5.7 & -2.8 & 2.2 & .2) & & & -39.6 \\
\hline 116 & azaquin & 1.000 & 6.3 & 8.7 & -1.8 & 3.7 & -4.6 & 3.4 & -9.8 & 1.6 & 10.1 & 6.9 & -3.6 & 5.6 & -1.6 & 5.4 & -7.2 & 4.2 & 94. & .8) & .7) & 3.3 \\
\hline 117 & azetha & 0.999 & -4.9 & 19.1 & 1.7 & 9.3 & -1.0 & 3.7 & -1.6 & 1.5 & -1.8 & 15.1 & -1.5 & 5.9 & 0.6 & 3.1 & -5.3 & 5.7 & $109.8(14.3)$ & $0.5(7.4)$ & $0.3(4.8)$ & -29.3 \\
\hline 118 & ole & 99 & 16.5 & 3.6 & 1.0 & 6.6 & -5.5 & 3.9 & -7.1 & 2.0 & -2.5 & 13.8 & -2.5 & 10.8 & -4.4 & 2.7 & -3.8 & 5.5 & .0) & .2) & .3) & -1.3 \\
\hline 119 & & 96 & -2.1 & $\begin{array}{l}0.0 \\
12.1\end{array}$ & 5.9 & 3.8 & 8.1 & 10.7 & 2.9 & 4.8 & 4.6 & 17.5 & 5.0 & 11.3 & 3.4 & 14.0 & $\begin{array}{l}-0.0 \\
-6.6\end{array}$ & & & & .9) & $\begin{array}{l}-1.0 \\
-46.7\end{array}$ \\
\hline 100 & & & -4.1 & 11. & $x_{0}$ & 5.5 & ( & No & 1 & s & & 12 & 5 & & & & s & & & & & -28.8 \\
\hline 12 & & & -8.2 & 12 & -3.2 & 5. & -3.5 & 6 & -8.6 & 4.4 & -10.1 & 17 & 2 & 7 & 5.9 & & -4.8 & & & & & 0.0 \\
\hline 122 & & & 6.3 & 12.7 & 0.0 & 4. & -4.3 & 2. & $\begin{array}{l}-0.9 \\
-3.9\end{array}$ & 2. & 7.0 & 14.5 & 2. & 3.4 & -2.7 & 8 & $\begin{array}{l}-4.0 \\
-4.1\end{array}$ & b. & $7.9(10.6)$ & .9) & .7) & $\begin{array}{l}4.9 \\
-9.8\end{array}$ \\
\hline 123 & Ip & & -2.8 & 9.8 & -2.2 & 3.2 & -5.4 & 1.2 & -8.7 & 2.4 & -6.9 & 7.4 & 0.0 & 7.4 & -2.3 & 3.9 & -4.1 & 4.2 & $90.1(1.0)$ & & .0) & -3.4 \\
\hline 124 & & & -8.2 & 5.6 & 5.7 & 2.6 & -2.2 & 3. & -8.9 & 1.9 & -11.1 & 2.7 & 6.0 & 5.8 & -2.5 & 2.7 & -6.9 & $-\pi$ & & & & -4.0 \\
\hline 125 & & & -1.0 & 4.6 & 0.0 & 2.0 & -2.0 & 0.1 & -4 & 1.9 & 3.5 & 7.3 & -0.1 & & -3.4 & & -4.0 & & & & & $\begin{array}{l}-4.0 \\
-23.9\end{array}$ \\
\hline 政 & & & -7.3 & 7 & & & & & & & & 7 & -1 & & & & & & & & & 8.3 \\
\hline 12 & & & s & & & & & & & & & & & & & & & & & & & 13.8 \\
\hline 128 & & & -7.1 & 10.7 & -1.0 & 4. & -4.6 & 2. & -11.9 & 3 & -8.9 & 17 & 2.5 & 10 & -0.8 & 6. & -7 & & & & & -0.7 \\
\hline 129 & & & -6.5 & 13 & 2.7 & 5. & -0.7 & 2. & -5 & 2. & 0. & 9.8 & 0 & 4.8 & -3.2 & 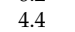 & -6.0 & 2 & & & & -1.4 \\
\hline 130 & esoy & & 7.2 & 12 & 4. & 7. & & & & 3 & -12.8 & 15 & 10.3 & 10.0 & 3.3 & & -5 & & & & & -11.9 \\
\hline 13 & & & -15.7 & 18 & -0.3 & 2.1 & - & 2 & - & 1. & - & 15 & -0.2 & 6. & & & -7 & & & & & -10.2 \\
\hline 13 & $M_{i}$ & & $\begin{array}{l}-1.9 \\
-1.9\end{array}$ & 6. & 0.8 & 2 & & 1. & & & $=$ & 10 & -0.2 & & 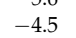 & & - & & & & & 78 \\
\hline 政 & & & 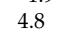 & 5 & 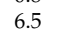 & & & & & & & 17 & & & & & & & & & & -5.0 \\
\hline 13 & & & -1 & 5 & & & & & & & & & & & & & & & & & & -6.9 \\
\hline 13 & feer & & 3. & 5 & 0 & 4. & 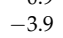 & 4 & & & - & I & & & & & & & & & & \\
\hline 13 & & & 12 & 10 & 9.2 & & & 2 & & 2. & 6 & 1 & 5.4 & & & & & 3 & & & & -4.7 \\
\hline 13 & & & 2. & 7. & 0 & & -4 & & & 1. & -4.7 & 12 & -2.7 & & $\begin{array}{lll}- & \\
-\end{array}$ & & -5 & & & & & -6.8 \\
\hline 13 & & & 10.7 & 3. & -4.2 & 9 & & & & & 5 & 12 & 1.1 & & -5 & & - & & 86 & & 76 & $\begin{array}{l}-0.0 \\
-6.1\end{array}$ \\
\hline 13 & & & -17.4 & 7. & $\begin{array}{l}-4.2 \\
-4.6\end{array}$ & 3 & & 3 & & 2 & $\begin{array}{c}5.4 \\
-13.2\end{array}$ & 11 & & & & & & & & & & $\begin{array}{l}-6.1 \\
65.1\end{array}$ \\
\hline 140 & & & -4.5 & 10 & -4 & 3 & & 2 & & 1 & 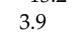 & 1 & - & & & & & & & & & -2.3 \\
\hline 14 & eth & & -0 & 4 & 0 & & & 1 & & 1 & & 6 & 1 & 3 & & & & & & & & \\
\hline 14 & & & $2+2$ & 16 & & & & 2 & & & & & & & & & & & & & & \\
\hline 14 & & & 0. & 9. & 3. & 6 & & 1. & -6 & 3. & - & 9 & 4 & & -1.2 & 2. & -6 & 4. & & & & -8.9 \\
\hline 144 & & 0.997 & 8.5 & 6.7 & 11.1 & 6.8 & 7. & 3. & -5.5 & 4.6 & 4.1 & 19.1 & 8 & 8 & 7.5 & 4. & -9.8 & 4.2 & $103.2(3.5)$ & $92.3(10.4)$ & $86.6(5.7)$ & -66.3 \\
\hline 145 & $\begin{array}{l}\text { Metho: } \\
\text { Mol }\end{array}$ & 0.997 & 10.2 & 4.8 & -2.3 & 7.6 & -8.7 & 5. & -13.1 & 4.1 & $\begin{array}{l}4.1 \\
3.3\end{array}$ & 19.6 & 2. & 6 & -1.1 & 6. & -7.2 & $\begin{array}{l}4.2 \\
6.8 \\
-12\end{array}$ & $71.9(5.8)$ & $106.1(6.1)$ & $5.3(6.4)$ & $\begin{array}{l}-0.3 \\
-6.8\end{array}$ \\
\hline No & & & to & 14 & 6. & & & 4. & & & & & & & & & & & & & & -31.8 \\
\hline 14 & & & 然 & 7 & -2 & 7.9 & & J. & & 3. & & & - & & & 5 & -2 & & & & & -3.2 \\
\hline 148 & $\mathrm{M}$ & 1.000 & -10.1 & 10.2 & 0.6 & 2.1 & -0.6 & 3.7 & -3.2 & 1.1 & -4.1 & 14.0 & 3. & 4.0 & -0.6 & 3.2 & -4.7 & 5.9 & $7.7(10.3)$ & $87.7(4.4)$ & $84.4(5.4)$ & -36.3 \\
\hline
\end{tabular}


Table 4. Cont.

\begin{tabular}{|c|c|c|c|c|c|c|c|c|c|c|c|c|c|c|c|c|c|c|c|c|c|c|}
\hline \multirow{3}{*}{ No. } & \multirow{3}{*}{ Compound Name } & \multirow{3}{*}{$r^{2}$} & \multicolumn{8}{|c|}{ Accuracy and Precision (Intra-Day) } & \multicolumn{8}{|c|}{ Accuracy and Precision (Inter-Day) } & \multicolumn{3}{|c|}{ Recovery, \% (RSD, \%) } & \multirow{3}{*}{$\begin{array}{l}\mathrm{ME}^{1} \\
\%\end{array}$} \\
\hline & & & \multicolumn{2}{|c|}{$10 \mathrm{ng} / \mathrm{mL}$} & \multicolumn{2}{|c|}{$50 \mathrm{ng} / \mathrm{mL}$} & \multicolumn{2}{|c|}{$150 \mathrm{ng} / \mathrm{mL}$} & $250 \mathrm{ng}$ & $\mathrm{g} / \mathrm{mL}$ & $10 \mathrm{ng}$ & //mL & $50 \mathrm{ng}$ & $/ \mathrm{mL}$ & $150 \mathrm{n}$ & $\mathrm{g} / \mathrm{mL}$ & $250 \mathrm{ng}$ & g/mL & & & 250 & \\
\hline & & & $\begin{array}{l}\text { RE } \\
\%\end{array}$ & $\begin{array}{c}\text { RSD } \\
\%\end{array}$ & $\begin{array}{l}\text { RE } \\
\%\end{array}$ & $\begin{array}{l}\text { RSD } \\
\%\end{array}$ & $\begin{array}{l}\text { RE } \\
\%\end{array}$ & $\begin{array}{c}\text { RSD } \\
\%\end{array}$ & $\begin{array}{l}\text { RE } \\
\%\end{array}$ & $\begin{array}{c}\text { RSD } \\
\%\end{array}$ & $\begin{array}{l}\text { RE } \\
\%\end{array}$ & $\begin{array}{c}\text { RSD } \\
\%\end{array}$ & $\begin{array}{l}\mathrm{RE} \\
\%\end{array}$ & $\begin{array}{c}\text { RSD } \\
\%\end{array}$ & $\begin{array}{l}\text { RE } \\
\%\end{array}$ & $\begin{array}{c}\text { RSD } \\
\%\end{array}$ & $\begin{array}{l}\text { RE } \\
\%\end{array}$ & $\begin{array}{c}\text { RSD } \\
\%\end{array}$ & & & & \\
\hline 149 & Metominostrobin & 1.000 & -0.2 & 3.9 & -1.7 & 3.0 & -5.5 & 4.2 & -7.6 & 1.4 & -4.0 & 12.9 & -1.6 & 3.2 & -2.8 & 1.9 & -4.5 & 4.0 & $87.6(4.1)$ & $90.6(3.5)$ & $81.5(5.2)$ & -11.9 \\
\hline 150 & Metrafenone & 1.000 & -5.8 & 12.3 & -1.7 & 4.7 & -2.9 & 1.6 & -6.2 & 0.6 & -1.5 & 14.2 & -1.3 & 7.1 & -2.6 & 3.6 & -5.8 & 8.9 & $77.8(9.3)$ & $88.2(11.0)$ & $76.8(5.6)$ & -0.7 \\
\hline 151 & Mevinphos & 00 & -3.8 & 6.4 & 8.4 & 2.2 & 3.2 & 2.3 & 1.4 & 1.3 & 3.7 & 11.0 & 1.3 & 7.2 & -0.8 & 3.0 & -3.6 & 5.0 & $80.6(1.5)$ & $90.7(1.8)$ & $3.6(4.0)$ & -47.6 \\
\hline 152 & Milbemectin A4 & 96 & -1.0 & 9.1 & -2.2 & 4.9 & -6.6 & 5.4 & -5.9 & 3.7 & 14.6 & 17.2 & -5.5 & 4.3 & -1.7 & 5.8 & 1.1 & 7.9 & $107.4(9.9)$ & $79.1(7.8)$ & $3.5(5.7)$ & -8.9 \\
\hline 153 & Mlinate & 1.000 & 17.1 & 3.5 & -2.5 & 3.3 & -8.9 & 2.8 & -8.9 & 2.8 & 9.3 & 12.0 & -0.8 & 4.5 & -2.3 & 3.0 & -5.6 & 5.9 & $91.9(5.6)$ & $90.6(9.8)$ & $.1(0.3)$ & 4.2 \\
\hline 154 & Monocrotophos & & -1.7 & 8.9 & 5.7 & 7.8 & 3.3 & 5.4 & -5.2 & 4.4 & -4.7 & & 7.1 & 5.9 & 4.2 & 2 & 75 & & $.6(16.6)$ & & & -60.1 \\
\hline 15 & Myclk & 96 & 4.1 & 5.0 & 13.1 & 9.1 & -0.4 & 2.2 & -7.8 & 3.7 & -12.5 & 15.2 & 14.9 & 7.0 & -0.7 & 2.5 & -6.8 & 9.2 & & 94.7 & 5) & 15.3 \\
\hline 156 & Propam & & 1. & 9.6 & -1.8 & 5.1 & -8.3 & 5.1 & -10.7 & 2.7 & -4.3 & 9.9 & -1.2 & 5.4 & -2.7 & 5.1 & -8.6 & 7.3 & 2) & 86.4 & .6) & -4.9 \\
\hline 157 & Nicosulfuron & 00 & 1.3 & 6.1 & -1.3 & 1.9 & -2.5 & 1.9 & -3.6 & 2.2 & 4.9 & 4.2 & -2.5 & 1.5 & -4.7 & 3.2 & -5.0 & 7.0 & .5) & 89.7 & $81.4(2.4)$ & -1.0 \\
\hline 158 & Nitenpyram & 992 & -8.3 & 6.6 & 10.7 & 8.1 & 1.1 & 5.4 & -7.0 & 7.5 & 5.7 & 19.9 & 6.1 & 5.9 & 2.0 & 3.6 & -8.4 & 5.8 & $85.5(8.3)$ & $88(8.3)$ & $76.1(11.9)$ & -15.3 \\
\hline 159 & ( & 399 & 11.8 & 18.5 & 7.1 & 5.9 & -4.6 & 4.9 & -4.6 & 2.8 & -0.7 & 15.7 & 1.1 & 4.6 & -4.9 & 10.0 & -3.4 & 6.4 & $95.6(12.9)$ & $83.6(12.9)$ & $75.8(4.1)$ & 13.0 \\
\hline 160 & Ofur & & 1.2 & 8.3 & 4.8 & 3.7 & -0.4 & 3.1 & -4.5 & 2.0 & 0.8 & (10. & $\begin{array}{l}1.1 \\
-1.2\end{array}$ & 5. & -1.4 & 10.0 & -5.6 & 0.7 & 11 & $2.2)$ & & -27.5 \\
\hline 10 & Omet & & 16.8 & 17.8 & 12.8 & 3 & 8.9 & 74 & 54 & 2 & -2.1 & 17 & 3.0 & 9 & -01 & 10.2 & 1 & & & & & -39.7 \\
\hline 162 & Orysastr & 00 & 4.4 & 4.2 & -0.1 & 1.5 & -4.8 & 3.6 & -6.5 & 2.1 & -2.9 & 7.3 & -4.7 & 5.6 & -4.6 & 3.4 & -5.6 & 3.9 & 6) & & & -0.4 \\
\hline 163 & Oxadiaz & & 0.2 & 15.6 & 11.8 & 6.0 & -1.5 & 7.1 & -8.9 & 2.3 & 12.8 & 19.9 & 9.4 & 6.9 & -4.8 & 5.7 & -1.9 & 7.6 & $97.5(16.4)$ & .0) & 6.8) & -4.3 \\
\hline 164 & Oxadixyl & 00 & -9.9 & 11.7 & 2.1 & 4.5 & 1.4 & 3.0 & -1.8 & 3.4 & -3.4 & 7.1 & 4.0 & 2.9 & 0.7 & 3.8 & -5.6 & 3.1 & $100(4.6)$ & 90.3 & 2.1) & -51.6 \\
\hline 165 & Oxamyl & 98 & 5.7 & 7.3 & 7.5 & 6.7 & 5.9 & 2.6 & -2.1 & 1.8 & 5.6 & 12.0 & 5.9 & 4.4 & 2.1 & 4.4 & -5.7 & 4.9 & $92.4(2.6)$ & $96(5.4)$ & 2.5) & -49.5 \\
\hline 166 & xydemeton-methyl & 0.998 & -6.4 & 6.0 & 3.6 & 2.8 & 1.9 & 3.2 & -6.3 & 18 & 0.1 & 7.1 & 1.8 & 4.0 & 1.9 & . & -6.0 & & & & & -48.8 \\
\hline 167 & & & -6.4 & 11.7 & & & & & & & -4.4 & & & & & & & & & & & .1 \\
\hline & & & -1 & 19. & -2 & & & & & & 13 & 15. & -7.3 & & 0. & & & & & & & \\
\hline 169 & Pencor & & -12.9 & 7.6 & 0.3 & 5.8 & -3.7 & 5.3 & -9.4 & 1.6 & 6.7 & 4.1 & 2.5 & 6.4 & 0.7 & 1.4 & -5.3 & 9. & $.1(10.8)$ & & $5.2)$ & -1.6 \\
\hline 170 & dimetl & & 2.3 & 15.6 & -5.2 & 2.6 & -4.2 & 2.9 & -5.7 & 1.7 & 10.0 & 13.9 & -4.9 & 2.8 & -2.8 & 2.2 & -1.8 & 8.3 & $86.6(7.1)$ & 80.9 (10.1) & 6.4) & -2.8 \\
\hline 171 & & & 1.9 & 5.9 & 3.8 & 2.6 & -1.3 & 2.5 & -5.3 & 1.1 & -3.7 & 12.0 & -0.8 & 5.2 & -2.1 & 4.6 & -5.9 & 8.6 & & & & 53.9 \\
\hline 17 & & & -11.0 & 9.9 & 5.7 & 9.0 & 0.7 & 2.8 & & 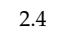 & 0.3 & & 7.7 & 2.7 & 1.8 & & & & & & & 0.4 \\
\hline 17 & & & -6.0 & 6 & 1 & 3. & -2 & & & & & o & & 2 & & & & & & & & 9 \\
\hline 174 & & & 2 & 1. & & 2 & 3 & & & & & & & & & & & & & & & 4.5 \\
\hline 175 & & & -16.6 & 7.5 & 6. & 5. & 0 & 2. & -4.6 & 3. & -13.6 & 10 & 6. & 7. & & 4.1 & -5.1 & 9. & & ) & & 0.0 \\
\hline 176 & & & -8.2 & 5.1 & 2. & 3.0 & -2 & 3.9 & & 2 & -9.3 & 16.4 & 2. & 2.6 & -2.2 & 3.1 & -5.6 & 4.9 & & & & -2.0 \\
\hline 177 & Phosp & & 12.4 & 4.3 & 0.5 & 2.9 & -0 & 2.1 & & 1 & 0.0 & 4.6 & 0 & 3.4 & -1 & 3.3 & -4.1 & 3.6 & & & & -29.4 \\
\hline 178 & & & -8.1 & 5.3 & 4.6 & 8.3 & -1 & 5.3 & & 0 & -16.5 & 13 & 4. & 6. & -5 & 3 & -7.1 & 10 & & & & -1.7 \\
\hline 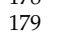 & & & -1 & 4.9 & 1. & 5.4 & -1 & & & 1 & -5.0 & & 5 & 6 & & & & & & & & -1.3 \\
\hline & & & -0 & a $>$ & & & & & & & & & & & & & & & & & & \\
\hline 18 & & & 5 & 6. & $-c$ & & & & & & & & -2 & & & & & & & & & 7 \\
\hline 182 & & & 6. & 3. & 1. & 2. & 0 & 1. & & 1 & & & 1.8 & 3 & -6 & 2 & - & & & & & -22.7 \\
\hline 183 & & & 4. & 6. & 0. & 5 & & & & 1 & -0.6 & 9.1 & -1.8 & 4. & -4 & 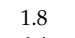 & & & & & & 0.5 \\
\hline & & & 8. & 12 & 1. & & & & & & 0.1 & 13 & 0 & 2.3 & & & & & & & & -3.7 \\
\hline 18 & & & 0.8 & 5. & -3.5 & 6. & & & & 1 & & & -0.7 & 5 & & & & & & & & -1.3 \\
\hline 18 & & & -16.5 & 10 & 5. & 7 & & & & 2 & & & 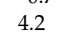 & & & & & & & & & -51.5 \\
\hline 18 & & & & & -8 & & & & & & & & & & & & & & & & & \\
\hline 18 & & & & 16 & 3 & & & & & & & & & & & & & & & & & \\
\hline 18 & & & 0. & 9 & - & & & & & & & & & & & & & & & & & -2.8 \\
\hline 19 & & & -5 & 6. & 4. & 3. & & & & & & & & & & 3. & & 6. & & & & -9.7 \\
\hline 13 & & & 1. & 5. & 6. & 6. & & 2. & - & 1. & -6.7 & 7.8 & J & & -1 & 3. & -4 & 4. & 5.6 & 86.8 & .2) & -12.2 \\
\hline 19 & & & 6. & 8 & 2 & & & 2. & & & & & & & & & & & & & & 4.4 \\
\hline 19 & & & -0.7 & 18 & & 8 & & & & & & 18 & & & & & & & & & & \\
\hline & & & +5 & 3 & & & & & & & & & & & & & & & & & & -30.0 \\
\hline 19 & & & -3 & 7 & - & & & & & & & & & & & & & & & & & -2.7 \\
\hline 19 & & & 9.1 & 6. & & & & & & 1. & 1 & & & 6.1 & & & & 12 & & & & -1.8 \\
\hline 197 & & & 8 & 3. & -1.8 & 5.1 & $-6 \quad r$ & 2.3 & -8 & 2.9 & 3.1 & 13.0 & -4.5 & 2.9 & -3.6 & 3.0 & -4.1 & 5.5 & $97.9(6.5)$ & $88.9(3.0)$ & $83.2(4.2)$ & 1.2 \\
\hline 198 & Py & 0.998 & 4.0 & 13.5 & 3.7 & 7.0 & -5.3 & 2.4 & -9 & 3.1 & 4.3 & 14.4 & 7.2 & 8.1 & 0.8 & 7. & -6.1 & 4. & $97(10.4)$ & 90.9 (16.5) & $73.5(3.9)$ & 3.5 \\
\hline 199 & & & -2.4 & 12.8 & 2.1 & 10.7 & -7 & 3.7 & & 5.3 & & 18 & -0.8 & 6. & & 8 & -2 & & & & & .0 \\
\hline 200 & & & -08 & 10. & 1.0 & 3. & $-2-2-1$ & 2. & & 2. & & 11.0 & -3 & 2.3 & -2 & 3.0 & & 84 & ) & 6) & $8.3)$ & 1.9 \\
\hline
\end{tabular}


Table 4. Cont.

\begin{tabular}{|c|c|c|c|c|c|c|c|c|c|c|c|c|c|c|c|c|c|c|c|c|c|c|}
\hline \multirow{3}{*}{ No. } & \multirow{3}{*}{ Compound Name } & \multirow{3}{*}{$r^{2}$} & \multicolumn{8}{|c|}{ Accuracy and Precision (Intra-Day) } & \multicolumn{8}{|c|}{ Accuracy and Precision (Inter-Day) } & \multicolumn{3}{|c|}{ Recovery, \% (RSD, \%) } & \multirow{3}{*}{$\underset{\%}{\mathrm{ME}^{1}}$} \\
\hline & & & \multicolumn{2}{|c|}{$10 \mathrm{ng} / \mathrm{mL}$} & \multicolumn{2}{|c|}{$50 \mathrm{ng} / \mathrm{mL}$} & \multicolumn{2}{|c|}{$150 \mathrm{ng} / \mathrm{mL}$} & \multicolumn{2}{|c|}{$250 \mathrm{ng} / \mathrm{mL}$} & $10 \mathrm{ng}$ & $/ \mathrm{mL}$ & $50 \mathrm{ng}$ & g/mL & $150 \mathrm{ng}$ & $\mathrm{g} / \mathrm{mL}$ & $250 \mathrm{ng}$ & $\mathrm{g} / \mathrm{mL}$ & & & 250 & \\
\hline & & & $\begin{array}{l}\mathrm{RE} \\
\%\end{array}$ & $\begin{array}{c}\text { RSD } \\
\%\end{array}$ & $\begin{array}{l}\mathrm{RE} \\
\%\end{array}$ & $\begin{array}{c}\text { RSD } \\
\%\end{array}$ & $\begin{array}{l}\text { RE } \\
\%\end{array}$ & $\begin{array}{c}\text { RSD } \\
\%\end{array}$ & $\begin{array}{l}\mathrm{RE} \\
\%\end{array}$ & $\begin{array}{c}\text { RSD } \\
\%\end{array}$ & $\begin{array}{l}\mathrm{RE} \\
\%\end{array}$ & $\underset{\%}{\text { RSD }}$ & $\begin{array}{l}\mathrm{RE} \\
\%\end{array}$ & $\underset{\%}{\text { RSD }}$ & $\begin{array}{l}\mathrm{RE} \\
\%\end{array}$ & $\begin{array}{c}\text { RSD } \\
\%\end{array}$ & $\begin{array}{l}\mathrm{RE} \\
\%\end{array}$ & $\begin{array}{c}\text { RSD } \\
\%\end{array}$ & & & & \\
\hline 201 & Pyribenzoxim & 0.996 & 9.4 & 9.4 & -14.3 & 13.2 & -12.2 & 4.8 & -11.7 & 4.8 & -5.8 & 10.6 & -11.5 & 12.4 & -6.9 & 6.8 & -2.2 & 11.2 & $92.2(4.5)$ & $71.8(17.8)$ & $85.8(6.9)$ & 1.7 \\
\hline 202 & Pyributicarb & 1.000 & 4.0 & 11.1 & -0.1 & 6.5 & -3.9 & 1.9 & -4.8 & 1.5 & -0.4 & 14.9 & 0.4 & 4.9 & -2.5 & 2.8 & -4.3 & 7.8 & $89.6(5.3)$ & $9.2(7.0)$ & $74.9(7.1)$ & 0.5 \\
\hline 203 & Pyridaben & 00 & 6.9 & 2.4 & -0.9 & 6.7 & -6.6 & 4.3 & -6.5 & 3.6 & 1.8 & 6.3 & -1.3 & 4.0 & -4.4 & 4.9 & -5.5 & 6.4 & $94.4(6.2)$ & $.4(6.9)$ & $82.1(5.2)$ & -5.0 \\
\hline 204 & Pyridalyl & 99 & 4.6 & 2.8 & -1.9 & 4.8 & -3.9 & 2.4 & -3.5 & 0.8 & 5.5 & 4.0 & -1.2 & 5.2 & -3.9 & 3.3 & -4.2 & 9.0 & $83.7(7.8)$ & $5.9(5.8)$ & $0.5(5.5)$ & 2.7 \\
\hline 205 & Pyridaphenthio & 0.997 & -12.8 & 15.2 & 1.8 & 6.1 & -3.7 & 5.0 & -9.2 & 3.5 & -7.4 & 9.0 & 72 & 4.8 & -4.3 & 25 & -9.2 & . & 94.9 (19.9) & $2.9(8.0)$ & $4.8(3.2)$ & 10.6 \\
\hline 206 & Pyridate & & 6.8 & 3.8 & -2.3 & 4.3 & -4.3 & 2.7 & -3.0 & 1.7 & 9.6 & 3.2 & -2.4 & 3.6 & & & & & & & & -0.5 \\
\hline 207 & Pyrifer & 0 & -3.8 & 8.9 & 4.9 & 10.8 & 7.0 & 2.3 & -9.6 & 4.1 & -10.1 & 11.5 & o. & 4.2 & & & & & & & & .8 \\
\hline 208 & iminobac- & 00 & 14.6 & 3.6 & 0.4 & 3.4 & -8.2 & 3.5 & -8.5 & $\begin{array}{l}4.1 \\
3.3\end{array}$ & 1.8 & 6.5 & -4.6 & 5.5 & -7.1 & 3.1 & -4.3 & .2 & 1) & .0) & .5) & 2.3 \\
\hline 209 & iminobac-methy & 1.000 & -0.9 & 3.8 & -1.1 & 3.3 & -5.9 & 2.6 & -5.7 & 1.1 & 4.7 & 7.2 & -3.4 & 3.8 & -5.3 & 5.1 & -4.9 & 5.3 & $103.9(8.7)$ & $5.0)$ & 3) & -7.0 \\
\hline 210 & imisulfan & 0.999 & 10.5 & 14.2 & 3.7 & 9.4 & -1.6 & 9.2 & -6.9 & 4.2 & -2.5 & 12.2 & 3.7 & 6.0 & 2.5 & 6.2 & -3.3 & 13.3 & 86 & $90.8(13.0)$ & $4)$ & 34.4 \\
\hline 211 & & 1.000 & -2.2 & 3.5 & 2.5 & 4.8 & -2.2 & 2.2 & -5.0 & 2.8 & -4.8 & 5.5 & 3.6 & 2.8 & -0.6 & 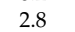 & -3.6 & 75 & & & & 0.1 \\
\hline 212 & roqui & & -10.5 & 6.1 & 4.2 & $\begin{array}{l}2.9 \\
2.9\end{array}$ & -2.2 & 2.4 & -2.4 & 2.0 & -2.3 & 14.1 & 0 & 2.0 & -2.9 & & -5.0 & 7. & & & & $\begin{array}{l}-3.1 \\
-34.7\end{array}$ \\
\hline 1 & & & -3.1 & 13.6 & -4.5 & 11.2 & 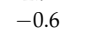 & 4 & -4 & 5 & 1 & 18 & 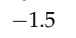 & & ? & & & & & & & -51.1 \\
\hline 21 & $\mathrm{R}$ & 9 & -4.8 & 10.2 & 2.3 & 2.7 & -7.5 & 3.3 & -9.5 & 2 . & -7.8 & 11.8 & 0.7 & 3.6 & -5.9 & 4 & -7.9 & 5.0 & & & & 30.1 \\
\hline 215 & ufen & & 11.0 & 8.1 & -3.8 & 6.4 & -7.9 & 2.3 & -11.5 & 4.9 & -0.6 & 13.9 & -5.2 & 8.4 & -4.7 & 4.6 & -5.9 & 9.0 & & .2) & 9) & 63.2 \\
\hline 216 & oxydim & & -13.1 & 4.8 & 8.3 & 4.6 & -2.4 & 3.8 & -6.3 & 2.3 & -3.8 & 16.5 & 5.4 & 2.9 & -2.8 & 5.4 & -5.9 & 7.4 & 6.8) & $3.1)$ & & -6.3 \\
\hline 217 & Simazi & 0.998 & -14.1 & 18.2 & 10.5 & 1.9 & 7.0 & 3.1 & 0.4 & 2.3 & -1.6 & 17.3 & 2.3 & 5.9 & 1.9 & 3.0 & -4.7 & 9.2 & 77.4 & 7.9) & & -34.8 \\
\hline 218 & Simecon & & 3.7 & 11.5 & 7.6 & 3.0 & -0.6 & S. & $\begin{array}{l}0.4 \\
-6.3\end{array}$ & 2.2 & -10.3 & 11.2 & 5. & & -1.5 & & -7.6 & & & & & 3.8 \\
\hline 1 & & & 2. & 7.4 & & 2.1 & & & -3 & 1 & & & 0 & & & & & & & & & 16.9 \\
\hline 220 & netora & & & 3.8 & . & 6.5 & -12.2 & & & & & & & & & & & & & & & . \\
\hline 221 & & & 3.3 & 4. & -4 & 6.7 & -8.7 & 4. & -6.8 & 3.4 & 6.0 & 9.8 & -5 & 5.9 & -4.3 & 5. & -4.2 & & & & & 5.1 \\
\hline 22 & oosy & & -6.4 & 3.7 & -1.3 & 2.6 & -5.3 & 3.1 & -5.5 & 1.4 & 17.4 & 9.4 & -3.3 & 8.3 & -5.3 & 2.8 & -4.2 & 6.6 & 106. & $86.2(10.0)$ & $1(4.5)$ & 2.5 \\
\hline 223 & & & 4.6 & 2.8 & 7.9 & 4.9 & -3.0 & 8.5 & -2.3 & 10.2 & -8.2 & 8.7 & 0.3 & 7.8 & -3.3 & 8.6 & -10.6 & 8.2 & & & & -2.3 \\
\hline 224 & & & 14.3 & 11.3 & 11.7 & & & & & & -2 & 8. & & & & & & & & & & -42.5 \\
\hline 22 & & & 5. & 10 & & 4 & & & & & & o & & & & & & & & & & 7.0 \\
\hline 22 & & & 10 & & 1. & & & & & & & & & & & & & & & & & 2.4 \\
\hline & & & 14 & 10 & 4. & 4.0 & & 2. & -7.1 & & & 13 & 0 & & -5 & & -5 & & & & & 5.7 \\
\hline 22 & & & 17 & 12 & 0. & 6. & - & 4. & -7 & 3 & -12.4 & 13. & 0 & 4 & -1.2 & 4.9 & -5 & 2 & & & & 8.8 \\
\hline 22 & & & 0.4 & 7. & 2. & 6.0 & & 2. & -6 & 2 & -1.4 & 9.5 & & 4. & -5 & 4.6 & -6.3 & & & & & -5.9 \\
\hline 23 & & & -3.6 & 7.1 & 5.2 & 7.1 & - & 3. & -7 & 1 & -1 & 5. & 6. & & -3 & & -10.6 & & & & & -16.6 \\
\hline 23 & & & -14.5 & 11.3 & -5.4 & & & & -7 & & & 15 & -4.6 & & & & -9.1 & & & & & 1.1 \\
\hline & & & 3 & & & & & & & & & & & & & & & & & & & \\
\hline & & & 10 & $1 \mathrm{c}$ & & & & & & & & & & & & & & & & & & .3 \\
\hline 23 & & & -8.6 & 6 & 8. & 4. & & 4 & & 4. & -2 & 7 & & & & & - & & & & & 8 \\
\hline 23 & & & 8. & 6 & & & & & & 2 & 9 & & . & & & & & & & & & -56.5 \\
\hline 23 & & & -18.3 & 14 & & & & & - & 3. & -5 & 13 & 4 & & & & & 4.9 & & & & -41.1 \\
\hline & & & 4.2 & 14 & 7. & & & & & & 10.4 & 13 & 4 & & & & & 12 & & & & -4.2 \\
\hline 23 & & & -6.6 & 10 & 3. & & & 3. & & 2. & & 10 & & & & & & & & & & 5.1 \\
\hline 23 & fen & & -1 & 4 & 2. & & & & & & & & & & & & & & & & & \\
\hline 24 & & & 1. & & & & & & & & & & & & & & & & & & & \\
\hline 24 & & & 8 & & & & & & & & & & & & & & & & & & & 9 \\
\hline 24 & & & 2 & & & & & & & & 6. & & & & & & & & & & & 42.1 \\
\hline & & & 2. & 12 & 0 & 5. & - & 2. & & 4. & 1 & 11 & & 6 & -3 & & - & 6.5 & 86. & 78 & 2) & -2.8 \\
\hline 24 & & & 13.7 & 17 & & 4. & & 3. & & & & 18.7 & & & & & & & & 94.7 & & 2.0 \\
\hline & & & 12.5 & 18 & -11.9 & 7 & & ? & & 7. & & 1 & 0 & & & & & & & & & 4 \\
\hline & & & - & 14 & -4 & & & & & & & 12 & - & & & & & & & & & \\
\hline & & & -4 & 6 & 2 & & & & & & & 14 & 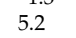 & & & & & & & & & \\
\hline & & & -3 & 2. & $-c$ & 1 & & 2 & & 1 & & 2 & - & & & 0 & & & & & & 18.0 \\
\hline 244 & & & -0 & 2. & 0. & 1. & -2.3 & 1.5 & -5 & 0.9 & -0.5 & 3. & 1 & 3. & -3. & 2. & -4.6 & 6.5 & & 91 & $77.4(2.3)$ & -1.4 \\
\hline 250 & Trichlorfon & 0.999 & 10.5 & 4.7 & 2.6 & 7.3 & 2.4 & 4.9 & -3.8 & 2.1 & 0.6 & 15.6 & 2.2 & 5.2 & -1.5 & 4.5 & -4.6 & 3.6 & 71.8 (15.5) & $88.8(7.3)$ & $81.1(5.2)$ & -32.1 \\
\hline
\end{tabular}


Table 4. Cont.

\begin{tabular}{|c|c|c|c|c|c|c|c|c|c|c|c|c|c|c|c|c|c|c|c|c|c|c|}
\hline \multirow{3}{*}{ No. } & \multirow{3}{*}{ Compound Name } & \multirow{3}{*}{$r^{2}$} & \multicolumn{8}{|c|}{ Accuracy and Precision (Intra-Day) } & \multicolumn{8}{|c|}{ Accuracy and Precision (Inter-Day) } & \multicolumn{3}{|c|}{ Recovery, \% (RSD, \%) } & \multirow{3}{*}{$\begin{array}{l}\mathrm{ME}^{1} \\
\%\end{array}$} \\
\hline & & & \multicolumn{2}{|c|}{$10 \mathrm{ng} / \mathrm{mL}$} & \multicolumn{2}{|c|}{$50 \mathrm{ng} / \mathrm{mL}$} & \multicolumn{2}{|c|}{$150 \mathrm{ng} / \mathrm{mL}$} & \multicolumn{2}{|c|}{$250 \mathrm{ng} / \mathrm{mL}$} & \multicolumn{2}{|c|}{$10 \mathrm{ng} / \mathrm{mL}$} & \multicolumn{2}{|c|}{$50 \mathrm{ng} / \mathrm{mL}$} & \multicolumn{2}{|c|}{$150 \mathrm{ng} / \mathrm{mL}$} & \multicolumn{2}{|c|}{$250 \mathrm{ng} / \mathrm{mL}$} & \multirow{2}{*}{$10 \mathrm{ng} / \mathrm{mL}$} & \multirow{2}{*}{$50 \mathrm{ng} / \mathrm{mL}$} & \multirow{2}{*}{$\begin{array}{c}250 \\
\mathrm{ng} / \mathrm{mL}\end{array}$} & \\
\hline & & & $\begin{array}{l}\mathrm{RE} \\
\%\end{array}$ & $\underset{\%}{\text { RSD }}$ & $\begin{array}{l}\mathrm{RE} \\
\%\end{array}$ & $\underset{\%}{\text { RSD }}$ & $\begin{array}{l}\mathrm{RE} \\
\%\end{array}$ & $\underset{\%}{\text { RSD }}$ & $\begin{array}{l}\mathrm{RE} \\
\%\end{array}$ & $\underset{\%}{\text { RSD }}$ & $\begin{array}{l}\mathrm{RE} \\
\%\end{array}$ & $\underset{\%}{\text { RSD }}$ & $\begin{array}{c}\mathrm{RE} \\
\%\end{array}$ & $\underset{\%}{\text { RSD }}$ & $\begin{array}{l}\mathrm{RE} \\
\%\end{array}$ & $\underset{\%}{\text { RSD }}$ & $\begin{array}{l}\mathrm{RE} \\
\%\end{array}$ & $\underset{\%}{\text { RSD }}$ & & & & \\
\hline 251 & Tricyclazole & 1.000 & 14.6 & 15.1 & 3.1 & 7.8 & 5.6 & 2.1 & -0.3 & 4.0 & 11.7 & 6.9 & -1.3 & 8.2 & 4.5 & 3.8 & -3.1 & 6.6 & $110.9(7.2)$ & $93(8.8)$ & $89.4(2.8)$ & -52.1 \\
\hline 252 & Trifloxystrobin & 0.999 & -5.0 & 5.3 & -0.2 & 5.8 & -7.1 & 1.8 & -9.1 & 2.7 & -3.5 & 13.6 & 4.0 & 6.6 & -2.2 & 4.9 & -6.3 & 5.5 & $81.5(3.8)$ & $89.5(8.4)$ & $78.4(6.6)$ & -5.9 \\
\hline 253 & Triflumizole & 0.999 & 12.9 & 10.1 & -1.8 & 5.3 & -5.4 & 2.3 & -6.5 & 1.3 & 1.2 & 17.8 & -4.1 & 4.8 & -3.0 & 5.1 & -3.2 & 7.8 & $90.7(11.7)$ & $83(6.0)$ & $76.9(10.2)$ & -4.0 \\
\hline 254 & Triflumuron & 0.996 & -8.3 & 9.6 & 10.2 & 7.2 & 1.1 & 4.1 & -9.1 & 3.6 & -13.0 & 8.6 & 5.5 & 4.6 & 1.0 & 5.6 & -5.0 & 10.2 & $85.9(7.8)$ & $91.1(4.2)$ & $74.7(8.7)$ & -5.8 \\
\hline 255 & Trimethacarb & 0.999 & -5.8 & 5.4 & 3.0 & 1.7 & -1.3 & 2.1 & -7.3 & 0.8 & -6.4 & 7.3 & 5.0 & 2.4 & -1.8 & 2.1 & -6.7 & 4.3 & $93.2(5.0)$ & $94.5(6.6)$ & $80.9(1.4)$ & -11.9 \\
\hline 256 & Triticonazole & 0.999 & $\begin{array}{l}-0.0 \\
-6.0\end{array}$ & $\begin{array}{l}3.4 \\
10.4\end{array}$ & $\begin{array}{l}.0 \\
0.7\end{array}$ & 1.9 & $\begin{array}{l}-1.5 \\
-3.9\end{array}$ & 4.8 & $\begin{array}{l}-1.5 \\
-8.2\end{array}$ & $\begin{array}{l}0.0 \\
2.8\end{array}$ & $\begin{array}{l}-0.4 \\
-4.5\end{array}$ & 10.5 & 3.5 & $\begin{array}{l}2.4 \\
6.5\end{array}$ & $\begin{array}{l}-1.0 \\
-5.0\end{array}$ & $\begin{array}{l}.1 \\
3.0\end{array}$ & $\begin{array}{l}-0.7 \\
-7.0\end{array}$ & $\begin{array}{l}4.5 \\
10.4\end{array}$ & $83(5.4)$ & $85.9(9.8)$ & $75.6(6.6)$ & 16.2 \\
\hline 257 & Uniconazole & 0.997 & -10.5 & 13.2 & 0.6 & 6.5 & -2.4 & 5.6 & -8.9 & 2.3 & -2.7 & 10.3 & 4.8 & 2.9 & -3.5 & 2.8 & -8.3 & 5.9 & $96.5(11.4)$ & $88.3(4.5)$ & $73.4(3.1)$ & 7 \\
\hline 258 & Vamidothi & 1.000 & 16.5 & 5.0 & 5.2 & 2.2 & -0.5 & 3.3 & -2.9 & 2.0 & 7.3 & 8.6 & 0.2 & 5.5 & -3.6 & 1.7 & -3.9 & 4.4 & $83(10.8)$ & $88.1(4.4)$ & $85(6.8)$ & -36.7 \\
\hline 259 & XMC & 1.000 & 7.3 & $\begin{array}{l}6.0 \\
6.45\end{array}$ & 4.4 & 2.8 & 1.7 & 1.8 & -0.9 & 3.0 & 1.3 & 11.5 & 2.5 & 4.4 & $\begin{array}{l}-1.0 \\
-1.4\end{array}$ & 3.6 & -3.5 & $\begin{array}{l}6.4 \\
6.6\end{array}$ & $95.2(5.8)$ & $\begin{array}{l}0.1+(7.4) \\
90(7.6)\end{array}$ & $81.2(5.9)$ & -21.4 \\
\hline 260 & Zoxamide & 0.999 & 8.6 & 7.4 & 3.1 & 3.6 & -5.2 & 4.9 & -9.3 & 1.8 & 1.2 & 14.7 & 2.4 & 3.1 & -4.0 & 5.6 & -6.1 & 6.7 & $96.7(6.6)$ & $85.7(9.3)$ & $76.8(2.0)$ & -4.4 \\
\hline
\end{tabular}




\subsubsection{Limit of Quantitation (LOQ) and Linearity of Calibration}

One of the methods for determining LOQ is to find the minimum concentration with a peak $\mathrm{S} / \mathrm{N}$ greater than 10 on the chromatogram [41]. It is easy but does not guarantee reproducibility in an analytical method. Therefore, relative error (RE, \%) and RSD in accuracy and precision study (Table 4) should be supplemented for the rugged LOQ validation. At $10 \mathrm{ng} / \mathrm{mL}$, target analytes were found to have an S/N > 10 as well as reasonable RE $(-18.4 \%$ to $19.5 \%)$ and RSD $(2.1 \%$ to $19.7 \%$ ) ranges. Those compounds with this concentration have sufficiently low detectability for various applications because urinary concentrations of parent compounds have been reported to range from sub to hundreds of $\mathrm{ng} / \mathrm{mL}$ in cases of acute pesticide intoxication or in some biomonitoring investigations $[17,20,26,42]$. Therefore, with the established analytical method, 260 pesticides can be determined in a urinary sample without further concentration of the sample extract.

Except for the target compounds, we have further studied more pesticides such as flonicamid and butocarboxim. They showed high LOQs (50 and $150 \mathrm{ng} / \mathrm{mL}$, respectively) in this methodology. The signal suppression by urine matrices were very strong for these compounds (matrix effect; $-58.8 \%$ and $-83.7 \%$, respectively), so further cleanup steps such as solid-phase extraction [43] are needed to remove matrices and to improve their LOQ levels.

The linearity of calibration is determined by a correlation coefficient $\left(r^{2}\right)$ of the 1 st order linear regression. The closer the $r^{2}$ value is to 1 , the better is the fit between signals and their concentrations (quantitative information). The 260 target pesticides showed $\mathrm{r}^{2} \geq 0.988$, meaning that target compounds had excellent quantitative properties with good linearity within their linear ranges.

\subsubsection{Accuracy and Precision}

In bioanalytical methodology, the accuracy is expressed as the RE of an expected value, and its variation in repeatability (precision) is determined using the RSD. According to the Guidance for Industry: Bioanalytical Method Validation criteria, acceptable accuracy ranges are divided into two types by treated levels; First, from $-20 \%$ to $20 \%$ with RSD $\leq 20 \%$ at LOQ level. Second, from $-15 \%$ to $15 \%$ with RSD $\leq 15 \%$ at other higher concentrations [44]. The accuracy and precision were validated at four treated levels of 10 (LOQ), 50, 150, and $250 \mathrm{ng} / \mathrm{mL}$ during the intra- and inter-day tests. For the concentration of $10 \mathrm{ng} / \mathrm{mL}$, RE ranges for 260 analytes were $-18.4 \%$ to $19.5 \%$ with RSDs from $2.1 \%$ to $19.7 \%$ in the intra-day measurements and $-18.2 \%$ to $17.4 \%$ with RSDs from $2.6 \%$ to $19.9 \%$ in inter-day (Table 4). For other concentrations $(50,150$, and $250 \mathrm{ng} / \mathrm{mL}$ ), RE ranges were $-14.7 \%$ to $13.1 \%$ with RSDs from $0.6 \%$ to $14.9 \%$ in the intra-day and $-11.5 \%$ to $14.9 \%$ with RSDs from $0.6 \%$ to $14.7 \%$ in inter-day. As a result, all the target compounds satisfied the accuracy and precision criteria. Although 260 pesticides were extracted together from a sample and analyzed simultaneously within only $15 \mathrm{~min}$, the analytes did not lose their chemical properties or react with each other. In addition, the MRM mode of the tandem mass spectrometry exhibited excellent throughput abilities to select, detect, and quantify hundreds of pesticides. Therefore, biomonitoring for pesticide multiresidues in urine can be determined with high reliability using this analytical method.

\subsubsection{Recovery}

The extraction efficiency of the preparation step is considered to be excellent when the recovery rate of a compound is close to $100 \%$. Therefore, recovery can affect the determination of sensitivity for target compounds. Recovery and its variation (RSD) are also regarded as accuracy and precision parameters in many bioanalytical methods [5,45]. Generally, a recovery rate of $70 \%-120 \%$ with RSD $\leq 20 \%$ is an acceptable range for those parameters [39]. The recovery study was conducted at treated levels of 10, 50, and $250 \mathrm{ng} / \mathrm{mL}$. The recovery ranges were $54.2 \%-113.9 \%$ (RSD; $0.5 \%-19.9 \%$ ), $71.8 \%-106.1 \%$ (RSD; $0.6 \%-20.0 \%$ ), and $68.5 \%-99.1 \%$ (RSD; $0.3 \%-11.9 \%$ ) at 10,50 , and $250 \mathrm{ng} / \mathrm{mL}$, respectively (Table 4). Most of the target pesticides satisfied the recovery range of $70 \%-120 \%$ with RSD $\leq 20 \%$ criteria. Only for the five compounds (chloridazon, chlorsulfuron, fluxapyroxad, milbemectin 
A4, and pretilachlor), lower recovery values $(54.2 \%-69.3 \%)$ were observed in some concentrations but acceptable repeatability (RSD; $5.7 \%-17.1 \%$ ).

From the recovery data, most of the pesticides showed high extraction efficiency by this bioanalytical method. In spite of the diverse chemical properties of the different pesticides, strong extraction/partitioning reagents make the pesticides maintain overall excellent recovery rates. Additionally, further cleanup steps were excluded to prevent the loss of target analytes.

Some compounds such as acetamiprid, cyprodinil, dicrotophos, imazalil, phosphamidon, and simazine showed higher recovery range $(77.4 \%-109.9 \%)$ at $10 \mathrm{ng} / \mathrm{mL}$ than that in Cazorla-Reyes et al. (2011)'s results $(60 \%-68 \%)$ in which analyzing 87 multiresidues using SPE (C18 cartridge) and LC-MS/MS [5]. It is considered that these compounds were adsorbed on C18 sorbent and some of them were not eluted.

For imidazolinones (imazapic, imazaquin, and imazethapyr), their recoveries were superior to those in our previous study using the same preparation in serum samples (Figure 2). Because imidazolinones are zwitterions, ion suppression is required using acidic buffers to improve extraction efficiencies in serum [35]. Urine, however, is generally acidic, so imidazolinones were fully extracted without the help of buffer reagents.

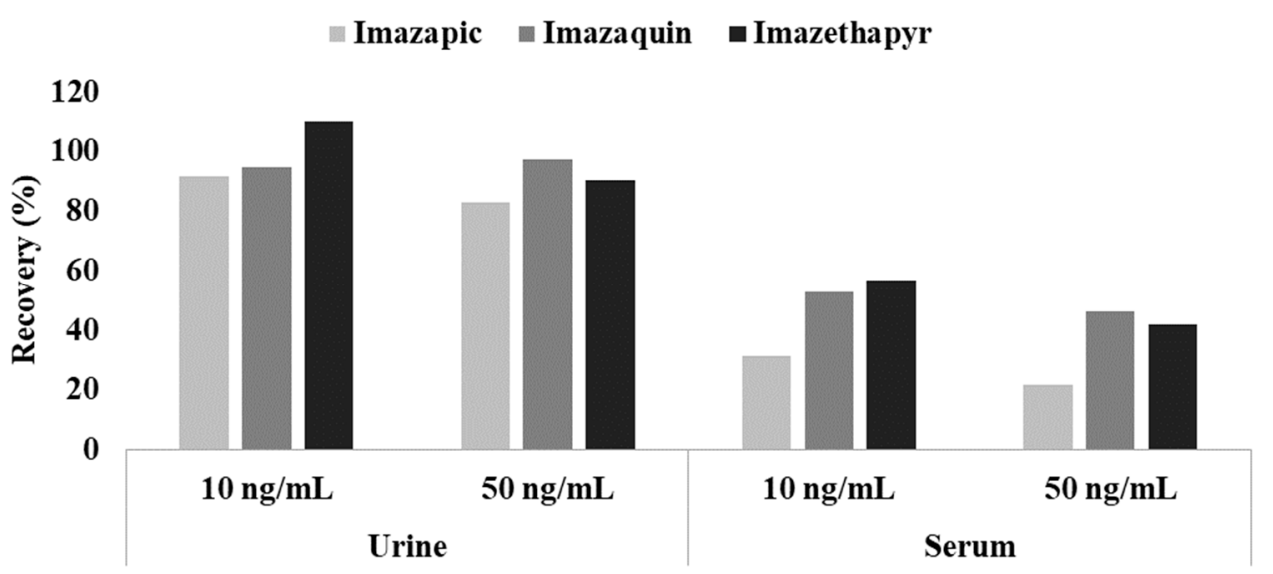

Figure 2. The recovery results for three imidazolinones in urine and serum samples.

Except for the target 260 compounds, we have further verified recoveries for more compounds such as dithianon, 4-trifluoromethylnicotinic acid (TFNA), and N-(4-trifluoromethylnicotinoyl)-glycine (TFNG) (metabolites of flonicamid) in urine. They showed poor recovery rates in this preparation procedure method $(35.2 \%-52.1 \%$ at 50 and $250 \mathrm{ng} / \mathrm{mL})$ as well as preparation method $\mathrm{B}$ and C. Dithianon is easily hydrolyzed in alkaline but stable in acidic media, thus dSPE including primary-secondary amine (PSA) is not suitable for urine treatment [46]. TFNA and TFNG need lower $\mathrm{pH}$ conditions for ion suppression, thus strong acidic reagents such as formic acid are needed to adjust $\mathrm{pH}$ and increase their recovery rates as well as LOQ level.

\subsubsection{Matrix Effect}

It has been reported that urine, as one of the most complex biological matrices, may affect ionization of target compounds in the ESI step of LC-MS/MS such that the intensity of the chromatogram could be enhanced or suppressed compared with that of non-matrix-based solutions $[47,48]$. Usually, the more concentrated is the urine, the more severe is the observed matrix effect [47]. Therefore, dilution of the sample is the common way to minimize the matrix effect and its mitigation between different samples $[16,49]$.

We have compared the matrix effects using the same extraction sovent volume ( $400 \mu \mathrm{L})$ but different urine volumes (100 $\mu \mathrm{L}$ vs. $400 \mu \mathrm{L})$. The overall matrix effects of target pesticides were reduced in smaller urine volume $(100 \mu \mathrm{L})$ (Figure S1). Therefore, $100 \mu \mathrm{L}$ of urine was selected with a four times 
larger volume ( $400 \mu \mathrm{L})$ of organic solvent to reduce the matrix effect. The extract was subjected to partitioning with salts $\left(\mathrm{MgSO}_{4}\right.$ and $\left.\mathrm{NaCl}\right)$ for the exclusion of polar compounds such as urea, salts, glucuronides, or sulfates from the organic layer to reduce the matrix effect. The percentage of matrix effect was calculated using the following Equation (1):

$$
\text { Matrix effect, } \%=\left(\frac{\text { Calibration slope of matrix based standard }}{\text { Calibration slope of solvent based standard }}-1\right) \times 100
$$

Therefore, matrix effect of each compound can be expressed as percentage enhancement $(>0 \%)$ or suppression $(<0 \%)$. The farther away the percentage is from zero $(0 \%)$, the larger is the matrix effect.

To summarize and evaluate matrix effects, the results of the 260 pesticides in Table 4 were classified into three groups defined by six ranges including a soft effect (matrix effect within $-20 \%$ to $0 \%$ or $0 \%$ to $20 \%$ ), medium effect ( $-50 \%$ to $-20 \%$ or $20 \%$ and $50 \%$ ), and strong effect (below $-50 \%$ or above $50 \%$ ) based on the reports of Kmellár et al. [50] and Ferrer et al. [51] (Figure 3).

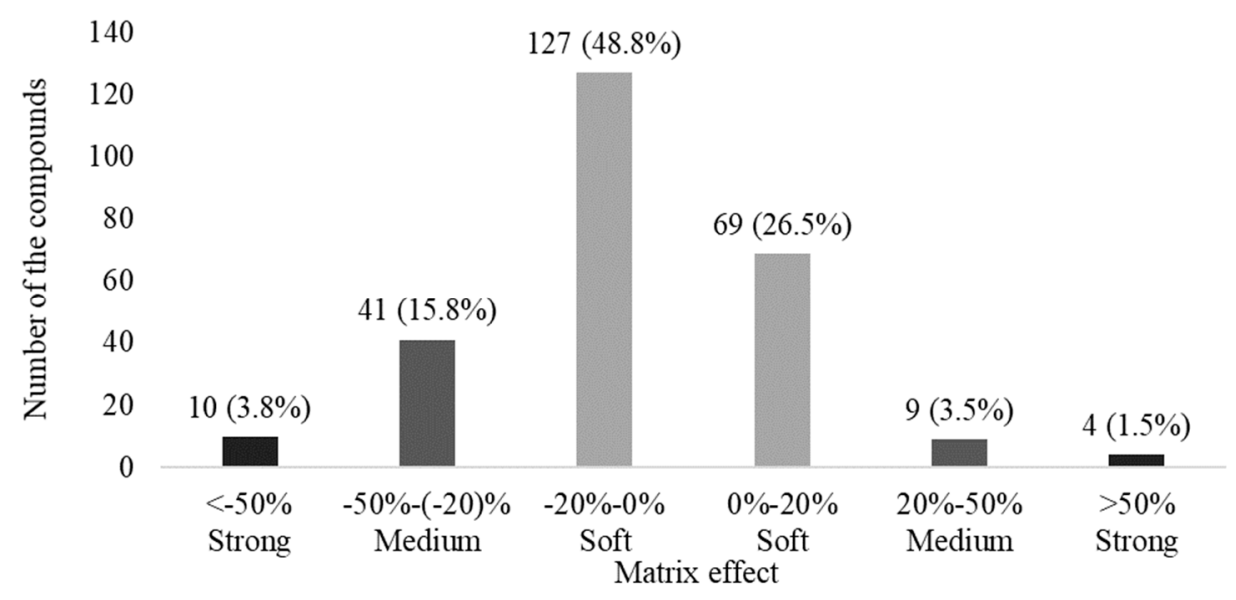

Figure 3. Summary of matrix effects for 260 pesticides in the final optimized method. Matrix effects are classified into soft effect (light grey bars, $-20 \%$ to $0 \%$ and $0 \%$ to $20 \%$ ), medium effect (grey bars, $-50 \%$ to $-20 \%$ and $20 \%$ to $50 \%$ ), and strong effect (dark grey bars, $<-50 \%$ and $>50 \%$ ).

Most of the pesticides (196, 75.3\% of total) were included in the soft effect group, in which 127 ( $48.8 \%$ ) compounds fell between $-20 \%$ and $0 \%$, and $69(26.5 \%)$ pesticides fell between $0 \%$ and $20 \%$. Within the soft group, matrix effects are considered negligible on LC-MS/MS [51]. Therefore, it is possible to determine the concentration of real urine samples using solvent-only (matrix-free) standard solution rather than matrix-matched solution. The numbers of compounds in the medium and strong groups were $50(19.3 \%)$ and $14(5.3 \%)$, respectively. These groups were susceptible to interfering influences in urine, thus requiring matrix-matched calibration for correct quantitation.

For verification of the matrix effect for each pesticide and correlation with retention time, a graph of matrix effects ordered by $t_{R}$ is shown in Figure 4. From the initiation time of pesticide elution to around $4.9 \mathrm{~min}$, a large number of pesticides showed the matrix effect below $-20 \%$. In contrast, during $t_{R}$ of 4.9 to $5.8 \mathrm{~min}$, signal suppression was reduced but some pesticides showed signal enhancement with matrix effects $>20 \%$. Matrix effects weakened after approximately $5.8 \mathrm{~min}$ through the end of the elution time. This result indicates that most of the polar urinary matrices co-eluted with target pesticides in the early stages of the analytical time ( $\sim 5.8 \mathrm{~min})$, causing considerable signal suppression/enhancement of target compounds. 


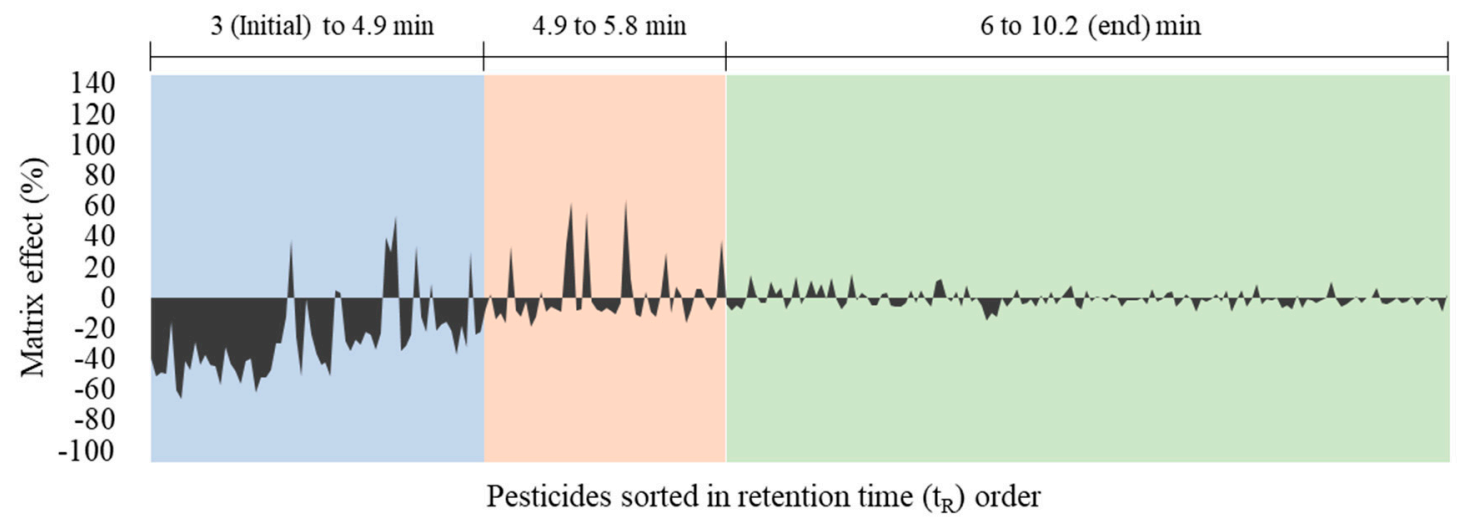

Figure 4. Distribution chart for 260 pesticides showing a pattern of matrix effects over retention time.

\subsection{Application}

Urine samples from agricultural workers were analyzed ( $n=10$, designated as \#1 to \#10) using the established method, and five samples (\#4,\#5,\#6,\#7, and \#10) showed positive detections (Table 5 and Figure 5). Therefore, this bioanalytical method with tandem mass spectrometry is appropriate to determine pesticides in unknown urine samples.

Table 5. Quantitative application results in urine samples obtained from agricultural workers.

\begin{tabular}{cccccc}
\hline Compound Name & \#4 ng/mL & \#5 ng/mL & \#6 ng/mL & \#7 ng/mL & \#10 ng/mL \\
\hline Imidacloprid & 11.7 & -1 & $<$ LOQ & 10.8 & - \\
Difenoconazole & - & 15.3 & - & - & - \\
Chlorfluazuron & - & - & - & - & $<$ LOQ \\
\hline
\end{tabular}

${ }^{1}$ Not detected.
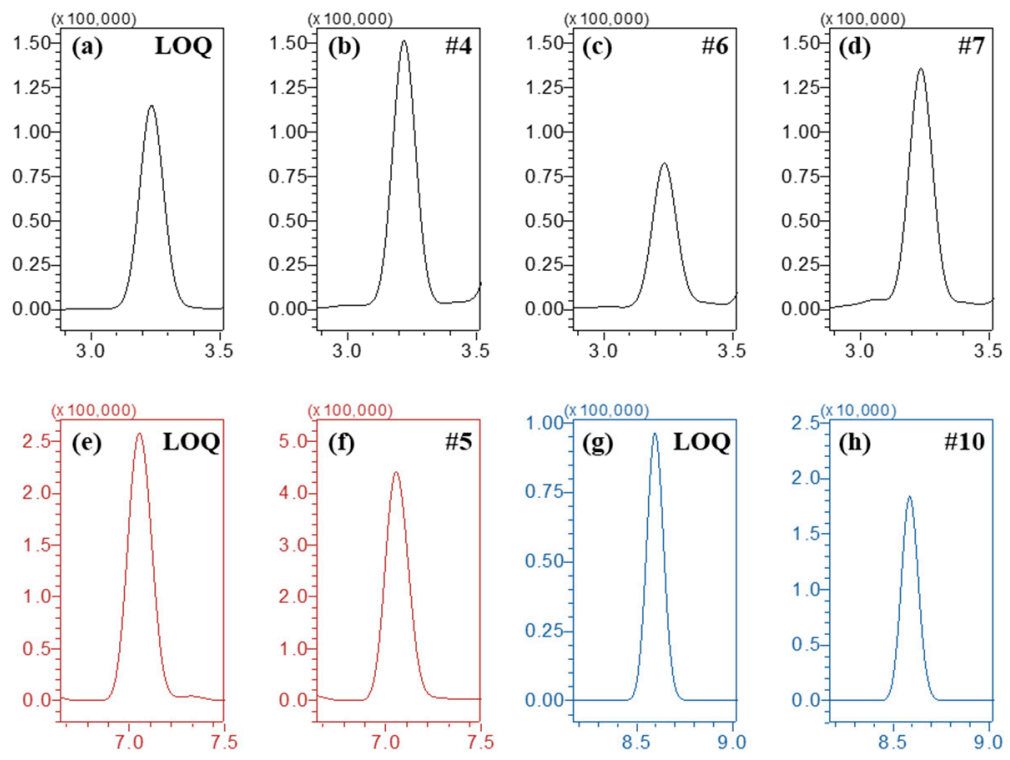

Figure 5. Chromatograms of (a-d) imidacloprid from QC (LOQ) and agricultural workers (\#4, \#6, and \#7), (e,f) difenoconazole from QC (LOQ) and agricultural workers (\#5), and (g,h) chlorfluazuron from QC (LOQ) and agricultural workers (\#10). MRM transitions in the chromatograms were $255.8>209.0$ (imidacloprid), $406.0>250.9$ (difenoconazole), and $539.8>382.8$ (chlorfluazuron), respectively. 


\section{Materials and Methods}

\subsection{Reagents}

Reference pesticide standards (purity $>98 \%$ ) or stock solutions $(1000 \mathrm{mg} / \mathrm{L}$ ) were sourced from Sigma-Aldrich (St, Louis, MO, USA), Dr. Ehrenstorfer (Augsburg, Germany), Wako Pure Chemical Industries (Osaka, Japan), ChemService (West Chester, PA, USA), and Ultra Scientific (North Kingstown, RI, USA). Acetonitrile and methanol (HPLC grade) were purchased from Fisher Scientific (Seoul, the Republic of Korea). Sodium chloride ( $\mathrm{NaCl}, 99.0 \%)$ was bought from Samchun (Gyeonggi-do, Korea). Ammonium formate ( $\geq 99.0 \%)$, formic acid (LC-MS grade), acetic acid $(\mathrm{HOAc}, \geq 99.7 \%)$, sodium citrate tribasic dihydrate $\left(\mathrm{Na}_{3}\right.$ Citrate $\left.\cdot 2 \mathrm{H}_{2} \mathrm{O}, \geq 99.0 \%\right)$, sodium citrate dibasic sesquihydrate $\left(\mathrm{Na}_{2} \mathrm{HCitr} \cdot 1.5 \mathrm{H}_{2} \mathrm{O}, \geq 99.0 \%\right)$, sodium acetate anhydrous ( $\left.\mathrm{NaOAc}, \geq 99.0 \%\right)$, and magnesium sulfate anhydrous $\left(\mathrm{MgSO}_{4}, \geq 99.5 \%\right)$ were obtained from Sigma-Aldrich. Ceramic homogenizers $(2 \mathrm{~mm}$ ) were obtained from Ultra Scientific. Ultrapure water was prepared in house using LaboStar ${ }^{\mathrm{TM}}$ TWF UV 7 (Siemens, Lowell, MA, USA).

\subsection{Urine Samples Collection}

Blank urine samples were obtained from healthy volunteers. For the application of the established method, ten samples from male agricultural workers were collected in urine bags. The samples were stored at $-70{ }^{\circ} \mathrm{C}$ until preparation and analysis. Urine sample collection was conducted under the permission of the Institutional Review Board (IRB) at Seoul National University, Seoul, the Republic of Korea (IRB No. 1604/002-007).

\subsection{Preparation of Standard Solutions}

Each reference standard was dissolved in acetonitrile, acetone, methanol, or water to prepare a $1000 \mathrm{mg} / \mathrm{L}$ stock solution. For some compounds (e.g., carbendazim) that are partially insoluble at this concentration, lower concentrations of stock solutions were prepared to make the compounds thoroughly soluble. These solutions were subjected to further dilution for use in MRM optimization on LC-MS/MS. To prepare four groups of intermediate mixed stock solutions at $10 \mathrm{mg} / \mathrm{L}$, a portion of each stock solution was brought up with acetonitrile in a 25-mL volumetric flask. The aliquots of intermediates were again mixed to make a final mixed standard solution at $2.5 \mathrm{mg} / \mathrm{L}$. This mixture was serial-diluted with acetonitrile for use in methodology validation steps. All the stock and standard solutions were stored at $-20^{\circ} \mathrm{C}$ until use.

\subsection{LC-MS/MS Instrumental Conditions}

LC-MS/MS analysis was carried out on a LCMS-8050 triple quadrupole mass spectrometer (Shimadzu, Kyoto, Japan) coupled to a Shimadzu Nexera X2 UHPLC system. For the mass spectrometer system, a heated ESI probe and positive/negative switching mode were used for target analyte ionization. Heating gas (air), drying gas (nitrogen), and nebulizing gas (nitrogen) flow rates were 10, 15, and $3 \mathrm{~L} / \mathrm{min}$, respectively. Argon gas was used for collision-induced dissociation (CID). The heat block, interface, and desolvation line (DL) temperatures were 400, 300, and $250{ }^{\circ} \mathrm{C}$, respectively. To optimize MRM conditions, each target analyte at $0.1-1 \mathrm{mg} / \mathrm{L}$ was subjected to a Q3 full scan with mass to charge ratio $(\mathrm{m} / \mathrm{z})$ range of $50-500$ or $100-1000$. A precursor ion (e.g., $[\mathrm{M}+\mathrm{H}]^{+}$) was selected according to its spectrum pattern, and more than 2 kinds of product ions were determined from the precursor ion using CID gas with variable collision energy (CE) voltages. Finally, two product ions with specific CEs were selected as quantifier and qualifier ions based on their selectivity and sensitivity. These optimized MRM conditions were scheduled according to the retention time of each compound, such that the MRM detection window was $\pm 0.5 \mathrm{~min}$. Dwell times were adjusted to $\geq 2.0 \mathrm{~ms}$ based upon loop time $(0.12 \mathrm{~s})$ for maximizing data acquisition.

The UHPLC system comprised a solvent delivery module (LC-30AD), column oven (CTO-20A), autosampler (SIL-30AC), and degassing unit (DGU-20A5R). A Kinetex ${ }^{\circledR}$ C18 column $(100 \times 2.1 \mathrm{~mm}$, 
2.6 $\mu \mathrm{m}$, Phenomenex, Torrance, CA, USA) was used for analyte separation, and a SecurityGuard ${ }^{\mathrm{TM}}$ Ultra guard column (Phenomenex) was connected to the column to prevent contamination. The oven temperature was maintained at $40{ }^{\circ} \mathrm{C}$. The total flow rate of the mobile phase was $0.2 \mathrm{~mL} / \mathrm{min}$. For the mobile phases, solvent $\mathrm{A}$ was $5 \mathrm{mM}$ ammonium formate and $0.1 \%$ formic acid in water and $\mathrm{B}$ was $5 \mathrm{mM}$ ammonium formate and $0.1 \%$ formic acid in methanol. For the gradient program, mobile phase B was initialized at $5 \%$, and maintained for $0.5 \mathrm{~min}$. The ratio of B was raised to $55 \%$ for $0.5 \mathrm{~min}$, ramped to $95 \%$ for $7 \mathrm{~min}$, held for $3 \mathrm{~min}$, raised to $100 \%$ for $1 \mathrm{~min}$, then dropped sharply to $5 \%$ for $0.1 \mathrm{~min}$, and held for $2.9 \mathrm{~min}$. The total analytical time was $15.0 \mathrm{~min}$, and the injection volume was $4 \mu \mathrm{L}$. LabSolutions software (version 5.72) was used for multiresidue MRM data processing.

\subsection{Preparation of Three Versions of QuEChERS}

The optimization of urine treatment methods by comparing three different versions of QuEChERS modified from Original [29], AOAC [30], and EN QuEChERS [31] was performed as follows: Method A; $400 \mu \mathrm{L}$ of acetonitrile, $40 \mathrm{mg}$ of $\mathrm{MgSO}_{4}$, and $10 \mathrm{mg}$ of $\mathrm{NaCl}$. Method B; $1 \%$ HOAc in acetonitrile $(400 \mu \mathrm{L}), 40 \mathrm{mg}$ of $\mathrm{MgSO}_{4}$, and $10 \mathrm{mg}$ of NaOAc. Method C; $400 \mu \mathrm{L}$ of acetonitrile, $40 \mathrm{mg}$ of $\mathrm{MgSO}_{4}$, $10 \mathrm{mg}$ of $\mathrm{NaCl}, 5 \mathrm{mg}$ of $\mathrm{Na}_{2} \mathrm{HCitr}$, and $10 \mathrm{mg}$ of $\mathrm{Na}_{3}$ Citrate. The extract from each method was centrifuged, and $200 \mu \mathrm{L}$ of supernatant was collected and mixed with $50 \mu \mathrm{L}$ of solvent (acetonitrile). The urine sample was equivalent to $200 \mu \mathrm{L}$ per $1000 \mu \mathrm{L}$ in the final extract. A portion of the sample $(4 \mu \mathrm{L})$ was injected into the LC-MS/MS, and the recovery as well as relative peak intensity of the three methods were compared to optimize the final sample preparation.

\subsection{The Final Optimized Method}

Human urine $(100 \mu \mathrm{L})$ was transferred to a $1.5-\mathrm{mL}$ microcentrifuge tube, and $400 \mu \mathrm{L}$ of acetonitrile and two ceramic homogenizers were added before being shaken with a Geno Grinder (1600 MiniG SPEX Sample Prep, Metuchen, NJ, USA) for $1 \mathrm{~min}$ at $1200 \mathrm{rpm}$. The sample was cooled in an ice bath and shaken again for $1 \mathrm{~min}$ at $1200 \mathrm{rpm}$ after $40 \mathrm{mg}$ of $\mathrm{MgSO}_{4}$ and $10 \mathrm{mg}$ of $\mathrm{NaCl}$ were added. After shaking, the sample was centrifuged for $5 \mathrm{~min}$ at $16800 \mathrm{~g}(13000 \mathrm{rpm})$ using a microcentrifuge (17TR, Hanil Science, Seoul, Korea). Then, $200 \mu \mathrm{L}$ of the upper organic layer was transferred to a 2-mL amber glass vial, and acetonitrile $(50 \mu \mathrm{L})$ was added for matrix-matching. Without further cleanup steps, the final extract $(4 \mu \mathrm{L})$ was injected into the LC-MS/MS for analysis of multiresidue pesticides.

\subsection{Analytical Method Validation}

The limit of quantitation (LOQ, $10 \mathrm{ng} / \mathrm{mL}$ ) was evaluated with signal to noise ratio $(\mathrm{s} / \mathrm{n}$ ) as well as RE and RSD of accuracy and precision results. The accuracy and precision tests were conducted on intra-day and inter-day conditions using a quality control (QC) sample (a sample with a known quantity of analyte [44]) with a calibration range from 10 to $250 \mathrm{ng} / \mathrm{mL}$. The intra-day test was performed in one day by analyzing five QC samples of urine at 10, 50, 150, or $250 \mathrm{ng} / \mathrm{mL}$, respectively. The inter-day test was carried out with a single QC sample of 10, 50, 150, or $250 \mathrm{ng} / \mathrm{mL}$ per day and repeated on 5 consecutive days. The accuracy and precision results were expressed with RE and RSD. For evaluation of the recovery, pesticides were spiked in blank urine before and after preparation $(10,50$, and $250 \mathrm{ng} / \mathrm{mL} ; n=3)$. The result was calculated as a ratio of the pre-spiking sample's response to the post-spiking sample's response. The matrix effect of each target compound was evaluated by comparing a calibration slope of matrix-based standard and that of solvent-based (matrix-free) standard.

\section{Conclusions}

A sensitive, fast, and simultaneous methodology for 260 pesticides in urine was successfully developed utilizing LC-MS/MS. Scheduled MRM for each target pesticide was optimized with the high-throughput triple quadrupole mass spectrometer. As a result, an average of 17.3 pesticides could be detected in a minute, thus the total analysis time for 260 pesticides was within only $15 \mathrm{~min}$ in 
a sample. Tiny volumes of urine $(100 \mu \mathrm{L})$ were used for sample preparation considering a realistic situation where a lot of urine cannot be collected from a pesticide poisoning victim or patient. To maximize extraction efficiency and minimize matrix effects, three versions of QuEChERS were compared, and the scaled-down QuEChERS procedure without dSPE cleanup was optimized for diverse chemical properties of the different pesticides. LOQs for target compounds were sufficiently low to detect pesticides in urinary samples. The final optimized analytical method for 260 pesticides was fully validated with the parameters of linearity of calibration, accuracy/precision, recovery, and matrix effect. The established method was successfully applied to determine pesticides in agricultural exposure samples. Therefore, the scaled-down QuEChERS method using LC-MS/MS can be a strong alternative to current analytical techniques.

Supplementary Materials: The following are available online at http:/ /www.mdpi.com/1420-3049/24/7/1330/ s1, Table S1: Recoveries and relative standard deviations (RSDs) of 260 pesticides for three versions of urine preparation methods; Table S2: Relative area intensities (100 at solvent standard peak area) of 260 pesticides for three versions of urine preparation methods; Figure S1: Matrix effects of 260 pesticides in $100 \mu \mathrm{L}$ and $400 \mu \mathrm{L}$ of urine samples.

Author Contributions: Conceptualization, Y.S., J.L., E.P., J.L. and J.-H.K.; experiments and data ananlysis Y.S., J.L., E.P. and J.L.; writing—original draft preparation, Y.S.; writing—review and editing, Y.S., J.L., H.S.L. and J.-H.K.; supervision, H.S.L and J.-H.K.; project administration, H.S.L.; funding acquisition, H.S.L.

Funding: This research was supported by the Bio and Medical Technology Development Program of the National Research Foundation (NRF) and funded by the Korean government (MSIP\&MOHW) (No. NRF-2015M3A9E1028325).

Conflicts of Interest: The authors declare no conflict of interest.

\section{References}

1. Cooper, J.; Dobson, H. The benefits of pesticides to mankind and the environment. Crop Prot. 2007, 26, 1337-1348. [CrossRef]

2. Gunnell, D.; Eddleston, M.; Phillips, M.R.; Konradsen, F. The global distribution of fatal pesticide self-poisoning: Systematic review. BMC Public Health 2007, 7, 357. [CrossRef]

3. Langley, R.L.; Mort, S.A. Human exposures to pesticides in the United States. J. Agromed. 2012, 17, 300-315. [CrossRef] [PubMed]

4. Cha, E.S.; Khang, Y.-H.; Lee, W.J. Mortality from and incidence of pesticide poisoning in South Korea: Findings from national death and health utilization data between 2006 and 2010. PLoS ONE 2014, 9, e95299. [CrossRef]

5. Cazorla-Reyes, R.; Fernández-Moreno, J.L.; Romero-González, R.; Frenich, A.G.; Vidal, J.L.M. Single solid phase extraction method for the simultaneous analysis of polar and non-polar pesticides in urine samples by gas chromatography and ultra high pressure liquid chromatography coupled to tandem mass spectrometry. Talanta 2011, 85, 183-196. [CrossRef] [PubMed]

6. Cortéjade, A.; Kiss, A.; Cren, C.; Vulliet, E.; Buleté, A. Development of an analytical method for the targeted screening and multi-residue quantification of environmental contaminants in urine by liquid chromatography coupled to high resolution mass spectrometry for evaluation of human exposures. Talanta 2016, 146, 694-706. [CrossRef] [PubMed]

7. Saito, T.; Fukushima, T.; Yui, Y.; Miyazaki, S.; Nakamoto, A.; Namera, A.; Inokuchi, S. Monolithic spin column extraction and GC-MS for the simultaneous assay of diquat, paraquat, and fenitrothion in human serum and urine. Anal. Bioanal. Chem. 2011, 400, 25-31. [CrossRef] [PubMed]

8. Takayasu, T.; Ishida, Y.; Nosaka, M.; Kawaguchi, M.; Kuninaka, Y.; Kimura, A.; Kondo, T. High concentration of methidathion detected in a fatal case of organophosphate-poisoning. Leg. Med. 2012, 14, 263-266. [CrossRef]

9. Venners, S.A.; Khoshnood, N.; Jeronimo, M.; Sobkowicz, A.; Provencher, P.; Tang, G.; Chu, W.; Copes, R. Adult and child urinary 2,4-D in cities with and without cosmetic pesticide bylaws: A population-based cross-sectional pilot study. J. Exposure Sci. Environ. Epidemiol. 2017, 27, 484-490. [CrossRef] 
10. Kasiotis, K.M.; Souki, H.; Carageorgiou, H.; Machera, K. Determination of dimethoate and omethoate in human serum samples. Risk assessment for the operator. Int. J. Environ. Anal. Chem. 2011, 91, 876-883. [CrossRef]

11. Papoutsis, I.; Mendonis, M.; Nikolaou, P.; Athanaselis, S.; Pistos, C.; Maravelias, C.; Spiliopoulou, C. Development and validation of a simple GC-MS method for the simultaneous determination of 11 anticholinesterase pesticides in blood—clinical and forensic toxicology applications. J. Forensic Sci. 2012, 57, 806-812. [CrossRef] [PubMed]

12. Sturza, J.; Silver, M.K.; Xu, L.; Li, M.; Mai, X.; Xia, Y.; Shao, J.; Lozoff, B.; Meeker, J. Prenatal exposure to multiple pesticides is associated with auditory brainstem response at 9 months in a cohort study of Chinese infants. Environ. Int. 2016, 92-93, 478-485. [CrossRef] [PubMed]

13. Hardy, E.M.; Duca, R.C.; Salquebre, G.; Appenzeller, B.M.R. Multi-residue analysis of organic pollutants in hair and urine for matrices comparison. Forensic Sci. Int. 2015, 249, 6-19. [CrossRef]

14. Schummer, C.; Salquèbre, G.; Briand, O.; Millet, M.; Appenzeller, B.M.R. Determination of farm workers' exposure to pesticides by hair analysis. Toxicol. Lett. 2012, 210, 203-210. [CrossRef] [PubMed]

15. Nigg, H.N.; Stamper, J.H.; Mallory, L.L. Quantification of human exposure to ethion using saliva. Chemosphere 1993, 26, 897-906. [CrossRef]

16. Hernández, F.; Sancho, J.V.; Pozo, O.J. Critical review of the application of liquid chromatography/mass spectrometry to the determination of pesticide residues in biological samples. Anal. Bioanal. Chem. 2005, 382, 934-946. [CrossRef]

17. Chen, S.; Zhang, Z.; He, F.; Yao, P.; Wu, Y.; Sun, J.; Liu, L.; Li, Q. An epidemiological study on occupational acute pyrethroid poisoning in cotton farmers. Br. J. Ind. Med. 1991, 48, 77-81. [CrossRef] [PubMed]

18. Draper, W.M. A multiresidue procedure for the determination and confirmation of acidic herbicide residues in human urine. J. Agric. Food Chem. 1982, 30, 227-231. [CrossRef]

19. Genuis, S.J.; Lane, K.; Birkholz, D. Human elimination of organochlorine pesticides: Blood, urine, and sweat study. Biomed Res. Int. 2016, 2016, 1624643. [CrossRef]

20. Montesano, M.A.; Olsson, A.O.; Kuklenyik, P.; Needham, L.L.; Bradman, A.; Barr, D.B. Method for determination of acephate, methamidophos, omethoate, dimethoate, ethylenethiourea and propylenethiourea in human urine using high-performance liquid chromatography-atmospheric pressure chemical ionization tandem mass spectrometry. J. Exposure Sci. Environ. Epidemiol. 2007, 17, 321-330. [CrossRef] [PubMed]

21. Lee, S.K.; Rhee, J.S.; Jung, J.M.; Lee, H.S. Pesticide poisoning deaths detected at the National Forensic Service Headquarters in Seoul of Korea: A five-year survey (2005-2009). Environ. Health Toxicol. 2010, 25, 263-271.

22. Turner, J.A. The Pesticide Manual: A World Compendium, 17th ed.; British Crop Production Council: Alton, UK, 2015.

23. Shin, Y.; Lee, J.; Kim, J.-H. A simultaneous multiresidue analysis for 203 pesticides in soybean using florisil solid-phase extraction and gas chromatography-tandem mass spectrometry. Appl. Biol. Chem. 2018, 61, 543-548. [CrossRef]

24. Esteve-Romero, J.; Marco-Peiro, S.; Rambla-Alegre, M.; Durgbanshi, A.; Bose, D.; Mourya, S.K. A micellar liquid chromatographic method for the determination of carbaryl and 1-naphthol in biological samples. J. Liq. Chromatogr. Relat. Technol. 2012, 35, 355-361. [CrossRef]

25. Kazui, Y.; Seto, Y.; Inoue, H. Phosphorus-specific determination of glyphosate, glufosinate, and their hydrolysis products in biological samples by liquid chromatography-inductively coupled plasma-mass spectrometry. Forensic Toxicol. 2014, 32, 317-322. [CrossRef]

26. Ueyama, J.; Nomura, H.; Kondo, T.; Saito, I.; Ito, Y.; Osaka, A.; Kamijima, M. Biological monitoring method for urinary neonicotinoid insecticides using LC-MS/MS and its application to Japanese adults. J. Occup. Health 2014, 56, 461-468. [CrossRef] [PubMed]

27. Watanabe, D.; Ohta, H.; Yamamuro, T. Solid-phase extraction of phosphorous-containing amino acid herbicides from biological specimens with a zirconia-coated silica cartridge. J. Chromatogr. B Anal. Technol. Biomed. Life Sci. 2014, 969, 69-76. [CrossRef]

28. Garner, F.; Jones, K. Biological monitoring for exposure to methamidophos: A human oral dosing study. Toxicol. Lett. 2014, 231, 277-281. [CrossRef] [PubMed] 
29. Anastassiades, M.; Lehotay, S.J.; Štajnbaher, D.; Schenck, F.J. Fast and easy multiresidue method employing acetonitrile extraction/partitioning and "dispersive solid-phase extraction" for the determination of pesticide residues in produce. J. AOAC Int. 2003, 86, 412-431.

30. Lehotay, S.J. Determination of pesticide residues in foods by acetonitrile extraction and partitioning with magnesium sulfate: Collaborative study. J. AOAC Int. 2007, 90, 485-520.

31. Foods of Plant Origin-Determination of Pesticide Residues Using GC-MS and/or LC-MS/MS Following Acetonitrile Extraction/Partitioning and Clean-Up by Dispersive SPE-QuEChERS-Method. 2008. Available online: https: / / refhub.elsevier.com/s0039-9140(19)30071-2/sbref33 (accessed on 5 March 2019).

32. Tuzimski, T.; Rejczak, T. A QuEChERS-based sample preparation method for the analysis of 5-nitroimidazoles in bovine milk by HPLC-DAD. J. AOAC Int. 2017, 100, 1671-1680. [CrossRef]

33. Rejczak, T.; Tuzimski, T. Method development for sulfonylurea herbicides analysis in rapeseed oil samples by HPLC-DAD: Comparison of zirconium-based sorbents and EMR-lipid for clean-up of QuEChERS extract. Food Anal. Method 2017, 10, 3666-3679. [CrossRef]

34. Plassmann, M.M.; Brack, W.; Krauss, M. Extending analysis of environmental pollutants in human urine towards screening for suspected compounds. J. Chromatogr. A 2015, 1394, 18-25. [CrossRef] [PubMed]

35. Shin, Y.; Lee, J.; Lee, J.; Lee, J.; Kim, E.; Liu, K.-H.; Lee, H.S.; Kim, J.-H. Validation of a multiresidue analysis method for 379 pesticides in human serum using liquid chromatography-tandem mass spectrometry. J. Agric. Food Chem. 2018, 66, 3550-3560. [CrossRef] [PubMed]

36. Tuzimski, T.; Pieniążek, D.; Buszewicz, G.; Teresiński, G. QuEChERS-based extraction procedures for the analysis of bisphenols $\mathrm{S}$ and A in breast milk samples by LC-QqQ-MS. J. AOAC Int. 2019, 102, $23-32$. [CrossRef]

37. Koesukwiwat, U.; Sanguankaew, K.; Leepipatpiboon, N. Rapid determination of phenoxy acid residues in rice by modified QuEChERS extraction and liquid chromatography-tandem mass spectrometry. Anal. Chim. Acta 2008, 626, 10-20. [CrossRef] [PubMed]

38. Ribeiro Begnini Konatu, F.; Breitkreitz, M.C.; Sales Fontes Jardim, I.C. Revisiting quick, easy, cheap, effective, rugged, and safe parameters for sample preparation in pesticide residue analysis of lettuce by liquid chromatography-tandem mass spectrometry. J. Chromatogr. A 2017, 1482, 11-22. [CrossRef] [PubMed]

39. Guidance Document on Analytical Quality Control and Validation Procedures for Pesticide Residues Analysis in Food and Feed SANTE/11813/2017. Available online: https: / ec.europa.eu/food/sites/food/ files/plant/docs/pesticides_mrl_guidelines_wrkdoc_2017-11813.pdf (accessed on 5 March 2019).

40. Malachová, A.; Sulyok, M.; Beltrán, E.; Berthiller, F.; Krska, R. Optimization and validation of a quantitative liquid chromatography-tandem mass spectrometric method covering 295 bacterial and fungal metabolites including all regulated mycotoxins in four model food matrices. J. Chromatogr. A 2014, 1362, 145-156. [CrossRef]

41. De Bièvre, P.; Günzler, H. (Eds.) Validation in Chemical Measurement; Springer: New York, NY, USA, 2005.

42. Usui, K.; Hayashizaki, Y.; Minagawa, T.; Hashiyada, M.; Nakano, A.; Funayama, M. Rapid determination of disulfoton and its oxidative metabolites in human whole blood and urine using QuEChERS extraction and liquid chromatography-tandem mass spectrometry. Leg. Med. 2012, 14, 309-316. [CrossRef]

43. Zhang, Q.; Wang, X.; Li, Z.; Jin, H.; Lu, Z.; Yu, C.; Huang, Y.-f.; Zhao, M. Simultaneous determination of nine neonicotinoids in human urine using isotope-dilution ultra-performance liquid chromatography-tandem mass spectrometry. Environ. Pollut. 2018, 240, 647-652. [CrossRef] [PubMed]

44. Bioanalytical Method Validation: Guidance for Industry. Available online: https://www.fda.gov/ downloads/drugs/guidances/ucm070107.pdf (accessed on 5 March 2019).

45. Kim, H.-S.; Kim, J.; Suh, J.H.; Han, S.B. General unknown screening for pesticides in whole blood and Korean gastric contents by liquid chromatography-tandem mass spectrometry. Arch. Pharm. Res. 2014, 37, 1317-1324. [CrossRef]

46. EURL-SRM-Analytical Method Report: Analysis of Dithianon in Food of Plant Origin Using Acidified QuEChERS and LC-MS/MS. Available online: http:/ / www.eurl-pesticides.eu/userfiles/file/EurlSRM/ meth_Dithianon_EurlSRM.pdf (accessed on 5 March 2019).

47. Panuwet, P.; Hunter, R.E.; D'Souza, P.E.; Chen, X.; Radford, S.A.; Cohen, J.R.; Marder, M.E.; Kartavenka, K.; Ryan, P.B.; Barr, D.B. Biological matrix effects in quantitative tandem mass spectrometry-based analytical methods: Advancing biomonitoring. Crit. Rev. Anal. Chem. 2016, 46, 93-105. [CrossRef] [PubMed] 
48. Schlittenbauer, L.; Seiwert, B.; Reemtsma, T. Matrix effects in human urine analysis using multi-targeted liquid chromatography-tandem mass spectrometry. J. Chromatogr. A 2015, 1415, 91-99. [CrossRef] [PubMed]

49. Ferrer, C.; Lozano, A.; Agüera, A.; Girón, A.J.; Fernández-Alba, A.R. Overcoming matrix effects using the dilution approach in multiresidue methods for fruits and vegetables. J. Chromatogr. A 2011, 1218, 7634-7639. [CrossRef] [PubMed]

50. Kmellár, B.; Fodor, P.; Pareja, L.; Ferrer, C.; Martínez-Uroz, M.A.; Valverde, A.; Fernandez-Alba, A.R. Validation and uncertainty study of a comprehensive list of 160 pesticide residues in multi-class vegetables by liquid chromatography-tandem mass spectrometry. J. Chromatogr. A 2008, 1215, 37-50. [CrossRef]

51. Ferrer, C.; Martínez-Bueno, M.J.; Lozano, A.; Fernández-Alba, A.R. Pesticide residue analysis of fruit juices by LC-MS/MS direct injection. One year pilot survey. Talanta 2011, 83, 1552-1561. [CrossRef]

Sample Availability: Not available.

(C) 2019 by the authors. Licensee MDPI, Basel, Switzerland. This article is an open access article distributed under the terms and conditions of the Creative Commons Attribution (CC BY) license (http:/ / creativecommons.org/licenses/by/4.0/). 